$32 / 22^{9185(2)}$

SSCL-500

Superconducting Super Collider Laboratory

8

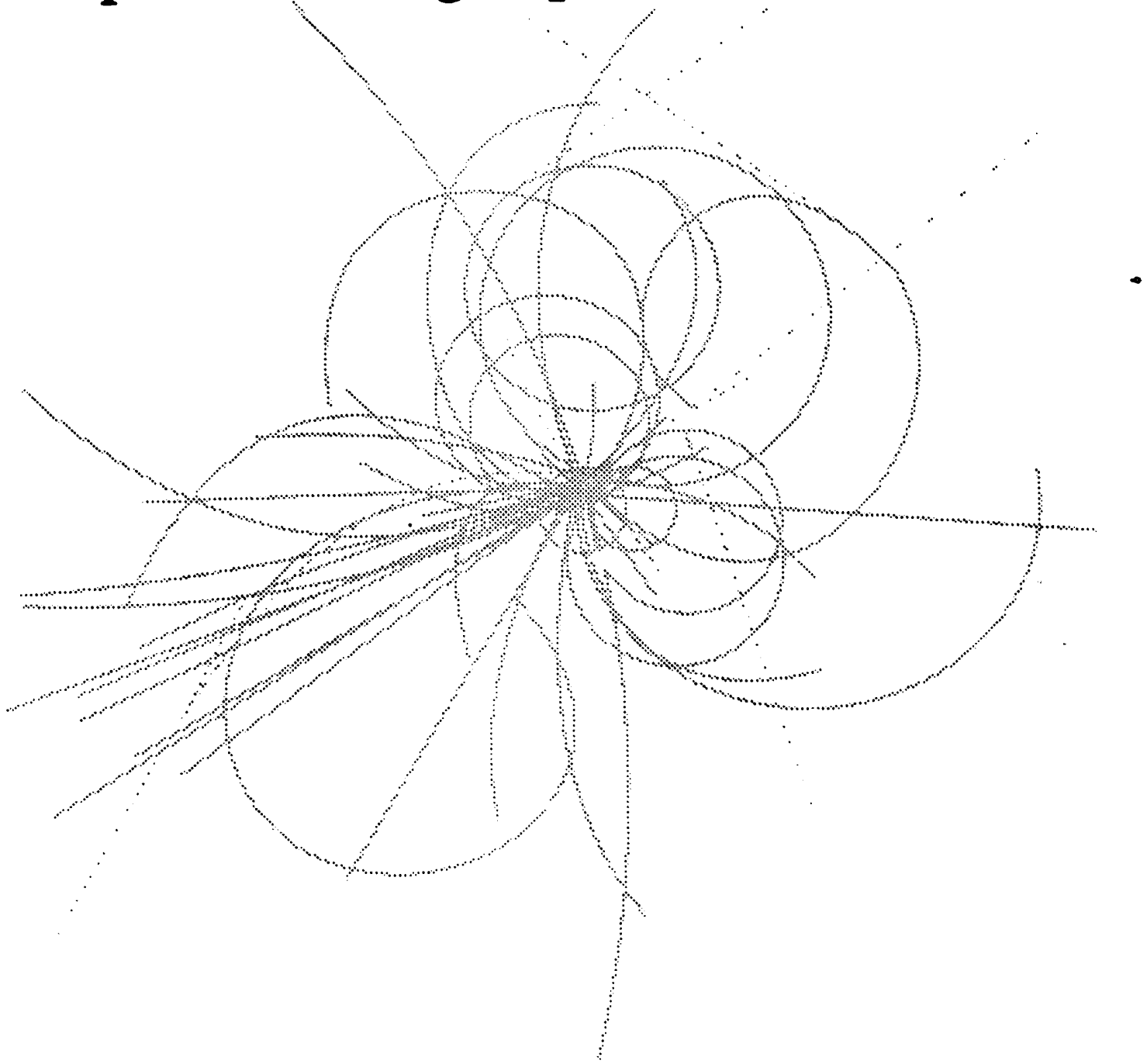

\title{
Applications of Differential Algebra to Single-Particle Dynamics in Storage Rings
}

\author{
Y. Yan \\ Accelerator Design and Operations
}

September 1991 


\title{
Applications of Differential Algebra to Single-Particle Dynamics in Storage Rings
}

\author{
Y. Yan \\ Accelerator Design and Operations \\ Superconducting Super Collider Laboratory* \\ 2550 Beckleymeade Avenue \\ Dallas, TX 75237
}

September 1991

DISCLAIMER

This report was prepared as an account of work sponsored by an agency of the United States Government. Neither the United States Government nor any agency thereof, nor any of their employees, makes any warranty, express or implied, or assumes any legal liability or responsibility for the accuracy, completeness, or usefulness of any information, apparatus, product, or process disclosed, or represents that its use would not infringe privately owned rights. Reference herein to any specific commercial product, process, or service by trade name, trademark, manufacturer, or otherwise does not necessarily constitute or imply its endorsement, recommendation, or favoring by the United States Government or any agency thereof. The views and opinions of authors expressed herein do not necessarily state or reflect those of the United States Government or any agency thereof.

* Operated by the Universities Research Association, Inc., for the U.S. Department of Energy under Contract No. DE-AC35-89ER40486. 


\title{
APPLICATIONS OF DIFFERENTIAL ALGEBRA TO SINGLE-PARTICLE DYNAMICS IN STORAGE RINGS
}

\author{
Yiton T. Yan \\ Superconducting Super Collider Laboratory \\ 2550 Beckleymeade Avenue, Dallas, TX 75237
}

\section{TABLE OF CONTENTS}

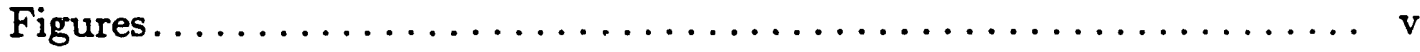

Tables................................. vi

1 Summary and Introduction $\ldots \ldots \ldots \ldots \ldots \ldots \ldots \ldots \ldots \ldots \ldots$

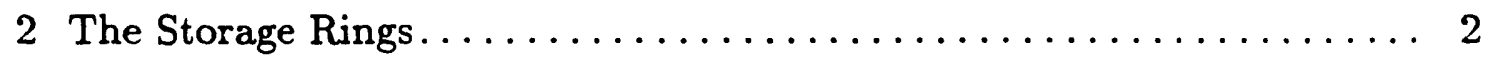

2.1 Betatron Resonances............................ 3

2.2 Synchro-Betatron Resonances...................... 3

2.3 Dispersions, Chromaticities, and Imperfections............ 4

2.4 Principles of Numerical Trackings.................... 5

2.5 Periodic Maps.............................. 5

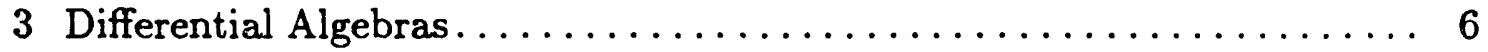

3.1 Symbolic Convention........................... 7

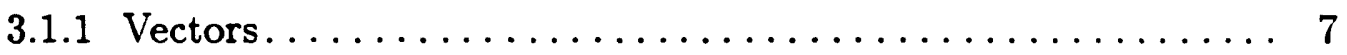

3.1 .2 Power Series........................... 8

3.1.3 Vector Power Series...................... 9

3.1.4 Numerical Implementations................... 10

3.1.5 Symplectic Identity . . . . . . . . . . . . . . . . . . 11

3.1 .6 Lie Operators.............................. 11

3.2 Power Series Operations........................... 12

3.2.1 Fundamental Power Series Operations............. 12

3.2.2 Basic Power Series Operations................... 13

3.3 Vector Power Series Operations....................... 14

3.3.1 Concatenation.............................. 14

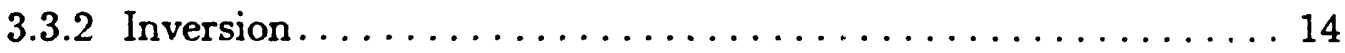

3.4 Global and Local Operators....................... 15

3.5 Theorems for Symplectic Maps and Lie Operators........... 16

3.6 Dragt-Finn Factorization......................... 17

3.7 Kick Factorizations.......................... 20

3.7.1 Irwin Kick Factorization Bases................ 22

3.7.2 Kick Factorization of a Homogeneous Lie Transformation . . 23 
3.7.3 Order-by-Order Kick Factorizations............. 25

3.7.4 Irwin's Minimization of the Spurious Terms . . . . . . 26

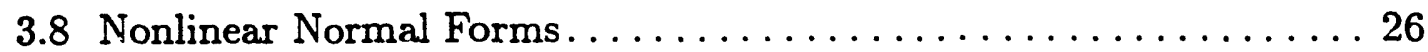

3.8.1 Order-by-Order Normalization. . . . . . . . . . . . 27

3.8.2 Eigenfunctions of the Linear Normal Form........... 29

3.8.3 Decomposition of the Homogeneous Polynomial......... 29

3.8.4 Nonlinear Resonances...................... 30

4 Long-Term Trackings.............................. 30

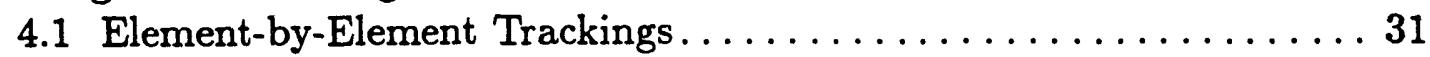

4.1 .1 Numerical Description........................ 31

4.1 .2 Survival Plots............................ 33

4.2 One-Turn-Map Trackings........................ 35

4.2.1 Construction of One-Turn Maps................ 35

4.2.2 The Taylor Maps............................ 39

4.2.3 Taylor Map Trackings..................... 40

4.2.4 Re-expanded Taylor Map Trackings............... 46

4.2.5 A Recipe for Taylor Map Trackings.............. 48

4.2.6 Kick-Map Trackings....................... 49

4.3 Multi-Turn-Map Trackings.......................... 49

5 Dispersed Betatron Motion......................... 51

5.1 The Parameterized Courant-Snyder Matrix.............. 52

5.2 Uncoupled Dispersed Betatron Motion................ 52

5.2.1 Normalization of the 0th-Order Courant-Snyder Matrix. . . . 53

5.2.2 Normalization of the 1st-Order Courant-Snyder Matrix . . . . 54

5.2.3 Normalization up to the $n$ th-Order Courant-Snyder Matrix. . 56

5.2.4 The Parameterized Twiss Parameters.............. 58

5.3 Coupled Dispersed Betatron Motion................... 60

5 Parameterization of One-Turn Maps.....................6. 62

6.1 Parameterized Lie Transformations.................. 63

6.1.1 Lie-Transformation Procedures.................6 64

6.1 .2 Further Justifications....................... 66

6.2 Faster Extraction of One-Turn 6-D Maps...............667

6.2.1 Conversion from 4-by-5 and 1-by-5 maps . . . . . . . . 68

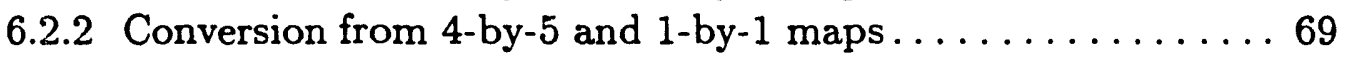

6.3 Parameterized Kick Factorizations................... 70

6.4 Parameterized Normal Forms $\ldots \ldots \ldots \ldots \ldots \ldots \ldots \ldots \ldots \ldots$

Acknowledgements................................ 74

References.................................. 75 


\section{FIGURES}

4.1 100,000-turn survival plots for two 2-TeV SSC injection lattices, comparing the dynamic aperture for two different magnet apertures-one with 4-cm-coil-diameter dipoles and the other with 5-cm-coil-diameter dipoles. With the 4-cm-coil-diameter dipoles, no particles with initial $x$ displacement amplitude of $<5.3 \mathrm{~mm}$ were lost. With the 5-cm-coil-diameter dipoles, no particles with initial $x$ displacement amplitude of $<8.1 \mathrm{~mm}$ were lost. By increasing the magnet aperture, the dynamic aperture for 100,000 turns enlarges from about $5.3 \mathrm{~mm}$ to about $8.1 \mathrm{~mm}$ in radius, and the machine's linearity improves. This plot shows only the protons that were lost before 100,000 turns are reached. . . . . . . . . . . 34

4.2 Turn-by-turn phase-space plots of an element-by-element tracking of the 5-cm-diameter dipole SSC injection lattice and its associated 12th-order Taylor map tracking for 400 turns. . . . . . . . . . . . 41

$4.310^{5}$-turn survival plots for a $2-\mathrm{TeV}, 5$-cm-diameter dipole injection lattice of the SSC, comparing the data from a 12th-order Taylor map (extracted with $\mathrm{Zmap}$ ) tracking with the data from its associated "Ztrack" element-by-element tracking. ................44

4.4 Survival plots for a 2-TeV, 5-cm-diameter dipole injection lattice of the SSC, comparing the data from the 12th-order Taylor map (extracted with $\mathrm{Zmap}$ ) tracking with the data from the 11th-order Taylor map tracking. . . . . . . . . . . . . . . . . . . . 43

4.5 Million-turn survival plots for the 2-TeV, 4-cm-diameter dipole injection lattice of the SSC, comparing the data from an 11th-order Taylor map (extracted with "Zmap") tracking with the data from its associated "Ztrack" element-by-element tracking. The two sets of data match quite well, showing that: (a) round-off errors (64-bit precision) for long-term tracking in the SSC are of no concern since "Ztrack" element-by-element tracking is accomplished at an accuracy of 11 digits in one turn, while the 11th-order Taylor map tracking is less accurate by 3 to 4 digits ( 7 to 8 digits of accuracy) in one turn for the selected region of interest, due to truncation of higher orders, with the same result; and (b) increasing the order of the Taylor map by one or two would allow reliable fast tracking up to 10 million turns for the SSC (the required proton-coasting time of the SSC injection lattice) since increasing one order in the Taylor map will deliver nearly 10 times as much accuracy (about one more digit accuracy) in one-turn tracking.

4.6 Error analysis of the one-turn Taylor map tracking for the $2-\mathrm{TeV}$, 4-cm-diameter dipole injection lattice of the SSC (the same lattice 
as used for Figure 4.5). The horizontal axis represents the transverse amplitude of the particles, while the vertical axis represents the weighted distance $D$ between the positions in the four-dimensional transverse phase space, obtained with the element-by-element tracking (Ztrack) and with the Taylor map tracking after one turn of each particle. $D=\left[\left(x^{Z}-x^{M}\right)^{2}+\beta_{x}^{2}\left(p_{x}^{Z}-p_{x}^{M}\right)^{2}+\left(\beta_{x} / \beta_{y}\right)\left(y^{Z}-y^{M}\right)^{2}+\right.$ $\left.\beta_{x} \beta_{y}\left(p_{y}^{Z}-p_{y}^{M}\right)^{2}\right]^{1 / 2}$, where $\partial \beta_{x} / \partial x=0=\partial \beta_{y} / \partial y$ at the measuring point. The deviation in energy for each of the particles is $\delta=4.2 \times$ $10^{-6}$. The region of interest for long-term tracking is around $0.005 \mathrm{~m}$ in trancverse amplitude, where the above figure shows the accuracy of the 11 th-order map to be between 7 and 8 digits after one turn. Note that for all the survival plots shown in this paper, all the particle initial energy deviations are about $\delta=5 \times 10^{-4}>4.2 \times 10^{-6}$. In such a case, the flat line at the lower left of the figure will be lifted and become longer. Nonetheless, the 7 to 8 digit accuracy at the region of interest is almost unchanged. ............... 45

4.7 Survival plots for a 2-TeV, 4-cm-diameter dipole injection lattice of the SSC, comparing the data from the 10th-order Taylor map tracking with the data from its associated element-by-element tracking.

4.8 Survival plots for a 2-TeV, 4-cm-diameter dipole injection lattice of the SSC, comparing the data from the re-expanded 11th-order and the re-expanded 12th-order (left-upper corner) Taylor map trackings (re-expanded from the 10th-order) with the data from its associated element-by-element tracking. . . . . . . . . . . . . . . . . 47

\section{TABLES}

3.1 Examples of 1-D Representations of a Vector Index $\vec{k} . \ldots \ldots \ldots \ldots$

4.1 Random, Systematic, and Orbit Errors and Their Corrections. . . . . 32 


\title{
APPLICATIONS OF DIFFERENTIAL ALGEBRA TO SINGLE-PARTICLE DYNAMICS IN STORAGE RINGS
}

\author{
Yiton T. Yan \\ Superconducting Super Collider Laboratory* \\ 2550 Beckleymeade Avenue, Dallas, TX 75237
}

\section{Summary and Introduction}

Recent developments in the use of differential algebra to study single-particle beam dynamics in charged particle storage rings are the subject of this paper. Chapter 2 gives a brief review of storage rings. The concepts of betatron motion and synchrotron motion, and their associated resonances, are introduced. Also introduced are the concepts of imperfections, such as off-momentum, misalignment, and random and systematic errors, and their associated corrections. The chapter concludes with a discussion of numerical simulation principles and the concept of one-turn periodic maps.

In Chapter 3, the discussion becomes more focused with the introduction of differential algebras. Symbolic conventions for vectors, power series, vector power series, and Lie operators are first established. The differential algebraic operations, including fundamental Lie transformations and their numerical implementations, are discussed. The concept of the Taylor map and its symplectification is introduced. Finally, the kick factorizations and the normal forms of the symplectic maps are discussed.

The most critical test for differential algebraic mapping techniques-their application to long-term stability studies-is discussed in Chapter 4. First, conventional element-by-element trackings are introduced, and a typical longterm tracking result-the survival plot-for the Superconducting Super Collider (SSC) is shown for subsequent comparisons with map-tracking results. Extraction and construction of one-turn maps are introduced, followed by discussion of one-turn map trackings. The success of long-term trackings using one-turn Taylor maps leads to an important conclusion for differential algebraic mappings: One-turn Taylor maps of moderate order can represent the SSC lattices accurately, and they can, in general, represent the lattices of other storage rings accurately, too.

* Operated by the Universities Research Association, Inc., for the U.S. Department of Energy under Contract No. DE-AC35-89ER40486. 
The requirement of symplecticity for long-term study is not a concern for the one-turn Taylor map. The Taylor map can always be symplectified via Lie transformations, which can be either re-expanded into a higher-order Taylor map to gain a higher degree of symplecticity, or converted into kicks (kicks are symplectic). Because map tracking is almost two orders of magnitude faster than conventional element-by-element tracking for the SSC, the author hopes that such conciusions will eventually encourage more popular use of differential algebraic maps for study of nonlinear dynamics.

Chapter 5 presents a discussion of differential algebraic treatment of dispersed betatron motion. The energy deviation is treated as a parameter for the transverse Courant-Snyder maps. Normalization via order-by-order symplectic factorization of the parameterized Courant-Snyder maps (matrices) leads to the parameterized betatron tunes and the parameterized Twiss parameters.

The paper concludes in Chapter 6 with a discussion of parameterization of high-order maps. The algorithm for parameterized Lie transformations of the parameterized Taylor maps is introduced. The key step leading to such parameterized Lie transformations is the normalization of the parameterized Courant-Snyder matrix. Once the parameterized Lie transformations are obtained for the one-turn transverse map, the 6-dimensional one-turn map-which includes the longitudinal motions-can be obtained through the conversion of the 4-dimensional parameterized Lie transformations. The parameterized kick factorizations and the parameterized normal forms can also be obtained.

\section{The Storage Rings}

A charged-particle storage ring is a structure within which charged particles with suitable energies are guided around a desired orbit so that the particles circulate without significant depletion for a period of time. Currently, the most popular structure is the strong-focusing synchrotron. ${ }^{1}$ The two collider rings of the SSC are the largest strong-focusing synchrotrons ever considered. ${ }^{2}$ They are designed to store and accelerate protons with an energy from $2 \mathrm{TeV}$ up to $20 \mathrm{TeV}$. A simple strong-focusing synchrotron consists of periodic arrays of dipole and quadrupole magnets along a desired circular orbit. As charged particles travel around the ring, the dipole magnets exert bending forces, thereby establishing the curvature of the orbit. The particles also experience focusing (or defocusing) forces from the quadrupoles, thus oscillating about the orbit. These oscillations are called betatron oscillations. This, fundamentally, is how charged particles are stored in a strong-focusing synchrotron; they are guided along the curved orbit by bending forces and are confined in the vicinity of the orbit, performing betatron oscillations due to the focusing forces. 


\subsection{Betatron Resonances}

The number of betatron oscillations for a particle traveling one turn is called the betatron tune, or simply the tune. The two betatron tunes associated with the two degrees of freedom transverse to the orbit are usually called the horizontal and vertical tunes. Since the synchrotron structure is naturally periodic in one turn, each of the tunes should be non-integral (not close to an integer); otherwise the betatron amplitude will grow resonantly with perturbations due to imperfections of the magnets. Similarly, the synchrotron is naturally periodic at other integer numbers of turns so that other deleterious resonances could also result. Thus, the number of betatron oscillations in two turns, three turns, and so on should be non-integer. Furthermore, there is usually coupling between the horizontal and vertical degrees of freedom and, therefore, resonance can also exist if the sum of the two numbers of horizontal and vertical betatron oscillations in one or a few turns is an integer. Denoting the fractional parts of the numbers of the betatron oscillations in one turn as $\nu_{x}$ and $\nu_{y}$ for the horizontal and the vertical tunes, respectively, these resonance conditions can be generally formulated as

$$
m \nu_{x}+n \nu_{y}=p
$$

where $r /, n$, and $p$ are integers and $|m|+|n|$ is the order of the resonance. Each order of resonance is driven by the corresponding order of perturbations-that is, the corresponding order of multipole errors in the magnets. Fortunately, resonance effect decreases as the order increases. Therefore, in designing the synchrotron, betatron tunes are chosen such that they are as remote as possible from the low-order resonances.

\subsection{Synchro-Betatron Resonances}

Storage rings are usually equipped with radio-frequency $(\mathrm{RF})$ cavities to support RF waves for maintaining the beam in bunches. Depending on the magnet excitation cycle, the RF fields can also accelerate particles. Through the principle 'st "phase stability," the RF fields induce longitudinal ("synchrotron") oscillations in the beam. That is, each beam particle in a bunch is made to oscillate in RF phase and in energy about the phase and energy of the ideal, central, synchronous particle in the bunch. The number of synchrotron oscillations in one turn is called the synchrotron tune. Because it is usually much smaller than 1, there are no pure synchrotron resonances that can be induced by the structure of the ring. Nonetheless, the synchrotron oscillations can modulate the betatron oscillations so as to produce "side-band" resonances. These additional resonances are called synchro-betatron resonances, and the general 
requirement for such resinnances is

$$
m \nu_{x}+n \nu_{y}+o \nu_{l}=p
$$

where $\nu_{l}$ is the synchrotron tune and again, $m, n, o$, and $p$ are all integers. In choosing the working betatron tunes $\nu_{x}$ and $\nu_{y}$, synchro-betatron resonances should also be taken into consideration.

\subsection{Dispersions, Chromaticities, and Imperfections}

There is always energy spread around the nominal energy in the particle beam. The energy spread causes closed-orbit spread around the nominal closed orbit, since particles with different energies receive slightly different deflections from the dipoles and the quadrupoles. ${ }^{*}$ This orbit difference is called dispersion. The dispersed closed-orbit deviation from the nominal closed orbit is a function of the longitudinal coordinate, $s$, and is dominated by the first-order effect of the energy deviation, which can be formulated as

$$
x_{c}(s)=D(s)\left(\Delta p / p_{0}\right)
$$

where $p_{0}$ is the magnitude of the nominal momentum, $\Delta p$ is the deviation in momentum magnitude, and $D(s)$ is the dispersion function.

The energy spread also causes tune spread around the nominal tunes because particles with different energies are focused differently in the quadrupoles. The tune shifts resulting from these "chromatic" effects are also basically dominated by the first-order effect of the energy deviation, which can be formuls. sed as

$$
\Delta \nu_{x}=\xi_{x}\left(\Delta p / p_{0}\right), \quad \Delta \nu_{y}=\xi_{y}\left(\Delta p / p_{0}\right)
$$

where $\xi_{x}$ and $\xi_{y}$ are the chromaticities of the machine. To reduce the chromatic tune shifts, insertion of correction sextupoles is required. Furthermore, imperfections such as misalignments and magnet multipole errors (both systematic and random) can distort the desired orbit and cause the betatron tunes to shift from the nominal tunes to unsatisfactory values. Correction dipoles and correction quadrupoles are also needed for the correction of the central orbit and

* For each given suitable energy, there exists an associated orbit such that a particle with that given energy can travel along the orbit turn after turn; that is, the orbit is closed on itself. A particle with a given energy but not with the right initial momentum and position does not travel on the closed orbit but performs betatron oscillation with respect to the closed orbit. A different given energy has a different closed orbit. 
the tunes, respectively. Therefore, in practice, a synchrotron-or a storage ring in general-is a complicated, nonlinear machine that makes detailed analytical study almost impossible. Computer simulations of tracking particle trajectories are thus widely employed.

\subsection{Principles of Numerical Trackings}

The various algorithms for tracking particles through a magnetic array comprising a storage ring involve calculating the magnetic forces due to all the component fields in each magnet. Each method of computation carries the presumption that for each magnet some very high-order (say, more than 9) multipole error effects are negligible. There is also the fundamental requirement that each of the algorithms preserve the symplectic property of the system. Through particle tracking, closed orbits, betatron tunes, and chromaticities can be analyzed and corrected by adjusting the correction dipoles, correction quadrupoles, and correction sextupoles. Often, the tracking programs are also equipped with schemes for the reduction of the multipole error effects, such as magnet-sorting algorithms. After correction, the full complicated lattice file is ready for tracking. Particles with given initial coordinates and momenta are tracked one element after another and, so, one turn after another. Most often, rays (particles' phasespace coordinates with respect to the desired orbit or the nominal closed orbit) at each turn are stored for post-analysis. Ultimately, a group of particles with well-chosen initial coordinates and momenta is tracked for a long term to study the dynamic aperture of the storage ring.

\subsection{Periodic Maps}

The periodicity of one turn is, of course, the main reason that turn-by-turin rays are most often used for analysis. Since the lattice file will not be changed again after the correction (assuming a static storage machine), it is also natural to think about a one-turn map. That is, at a fixed reference position around the ring, the rays at any given turn are related to the rays of the previous turn by a mathematical formula which, for the time being, is simply denoted as

$$
\vec{z}^{\prime}=m \vec{z},
$$

where $\vec{z}$ is a vector representing the rays at the given turn; $\vec{z}^{\prime}$ is a vector representing the rays at the next turn; and $m$ can be considered as a simplified operator representing the full complicated lattice file of the machine. Usually, $m$ is a functional form of $\vec{z}$, where $\vec{z}$ represents the 6 -dimensional phase-space coordinates; that is, the transpose of $\vec{z}$ is given by

$$
\vec{z}^{T}=\left(z_{1}, z_{2}, z_{3}, z_{4}, z_{5}, z_{6}\right)=\left(x, p_{x}, y, p_{y}, \tau,-\delta\right),
$$


where $x, y$ are the transverse coordinates with respect to the desired orbit or the closed orbit, and $p_{x}, p_{y}$ are their corresponding conjugate momenta, respectively; $\tau$ is usually called time of flight, which represents the longitudinal deviation from the synchronized position; and $\delta$ is the normalized energy deviation and is denoted as $\delta=\Delta E / E_{0}$. Note that $\tau$ is also normalized such that $-\hat{o}$ is its momentum conjugate.

If there is no synchrotron oscillation or if the RF cavity is separately treated, then the one-turn map is usually written as

$$
\vec{x}^{\prime}=m \vec{x},
$$

and the simplified operator, $m$, is usually a functional form of $\vec{x}$ and $\delta$, where $\delta$ is a parameter representing the normalized energy deviation and $\vec{x}$ represents the transverse phase-space coordinates; that is, the transpose of $\vec{x}$ is given by

$$
\vec{x}^{T}=\left(x_{1}, x_{2}, x_{3}, x_{4}\right)=\left(x, p_{x}, y, p_{y}\right) .
$$

If, indeed, a simplified form of the map $m$ can be obtained efficiently, and it can specify the full complicated lattice file accurately and can be sufficiently symplectic, it is clear that the turn-by-turn tracking can be made quite fast, and many of the machine properties can be easily analyzed. Thanks to the introduction by Berz of differential algebras to the accelerator field, ${ }^{3}$ a simple one-turn map-the Taylor map-can be obtained efficiently for a complicated periodic system. Also, it is thanks to Dragt and Finn that more applications of the map are possible through Lie transformations of the Taylor map of a symplectic system. ${ }^{4}$ Before discussing these issues further, we shall discuss differential algebras and the relevant Lie algebras in Chapter 3.

\section{Differential Aigebras}

Differential algebras arise from the field of non-standard analysis dealing with arbitrarily small quantities ${ }^{5,6}$ with useful applications to automatic differentiation and power series representation of analytical functions. ${ }^{7,8}$ In this paper, we have no intention to discuss differential algebras in general. Since physicists are quite familiar with the power series expansion, for the sake of simplicity and clarity we shall simply consider it as the algebra of power series in the domain within which the power series converge. Similar to many numerical libraries which perform linear algebra through matrix operations, there are numerical packages or libraries ${ }^{9-11}$ which perfor $n$ differential algebra through the operations of expanded power series-truncated at a pre-set order-to include nonlinear effects. Unlike linear algebra, which has a domain idealized to be 
unlimited, differential algebra has a narrow domain where the power series converge at a reasonable rate; that is, the scope of differential algebra is restricted to problems for which a region of interest can be identified to have a reasonable convergent rate for the power series expansion of the governing equations. Particle dynamics in a storaje ring fit this requirement, since particles will not likely survive in a region where the power series expansion of the governing equations diverges or converges very slowly.

We shall proceed to discuss the algebra of power series in this chapter without trying to be mathematically rigorous. Existence is assumed for all variables, functions, and oprrations that are mentioned.

\subsection{Symbolic Convention}

Detailed mathematical formulas for multi-variable power series expansions and their related operations are very lengthy. It is the author's intention in this section to establish a systematic symbolic convention so that the algebra of power series can be handled with less ambiguity.

\subsubsection{Vectors}

Let $\vec{z}$ be an $n$-dimensional vector; i.e., its transpose can be expressed as

$$
\vec{z}^{T}=\left(z_{1}, z_{2}, \ldots, z_{n}\right) \text {, }
$$

where $z_{i}$, for $i=1, \ldots, n$, are scalar variables. For example, we can consider

$$
\vec{z}^{T}=\left(z_{1}, z_{2}, z_{3}, z_{4}, z_{5}, z_{6}\right)=\left(x, p_{x}, y, p_{y}, \tau,-\delta\right)
$$

as the transpose of a vector representing the 6-dimensional phase-space coordinates for a storage ring. We will take the convention given by Eq. (3.1) that if the phase-space coordinates are represented by a vector, each of the conjugate coordinates takes an odd sequential position while its conjugate momentum is positioned right after.

An $n$-dimensional vector index $\vec{k}$ is an $n$-dimensional vector with all integer components that are larger than or equal to 0 ; that is, $\vec{k}^{T}=\left(k_{1}, k_{2}, \ldots, k_{n}\right)$, where $k_{i} \in I$ and $k_{i} \geq 0$. Associated with each $n$-dimensional vector index $\vec{k}$ is a vector index order $k$ given by

$$
k=\sum_{i=1}^{n} k_{i}
$$

Given two $n$-dimensional vector indexes $\vec{j}$ and $\vec{k}$, the following conventions are used throughout this paper:

$$
\vec{j} \geq \vec{k} \quad \text { if } \quad j: \geq k ; \quad \forall \quad i=1,2, \ldots, n
$$




$$
\vec{j} \leq \vec{k} \quad \text { if } \quad j_{i} \leq k_{i} \quad \forall \quad i=1,2, \ldots, n .
$$

A unit vector in the $i$ th dimension is denoted as $\vec{l}_{i}$. For example,

$$
\vec{I}_{1}=(1,0, \ldots, 0), \quad \vec{I}_{n}=(0, \ldots, 0,1) .
$$

\subsubsection{Power Series}

Let $U$ be a function of $\vec{z}$. This means $U$ is a function of $z_{1}, z_{2}, \ldots, z_{n}$. Its power series (PS) expansion up to an integer $\Omega$ order is expressed as

$$
U(\vec{z})=\sum_{k=0}^{\Omega} u(\vec{k}) \vec{z}^{\vec{k}}
$$

where

$$
\vec{z}^{\vec{k}} \equiv z_{1}^{k_{1}} z_{2}^{k_{2}} \ldots z_{n}^{k_{n}}
$$

and

$$
\sum_{k=0}^{\Omega} \equiv \text { summation over all indexes } \vec{k} \text { 's } \quad \forall \quad k=0,1, \ldots, \Omega .
$$

Note that, in general, we shall denote

$$
\begin{array}{lll}
\sum_{k=l} \equiv \text { summation over all indexes } \vec{k} \text { 's } & \forall \quad k=l, \\
\sum_{k=l}^{\Omega} \equiv \text { summation over all indexes } \vec{k} \text { 's } & \forall & k=l, l+1, \ldots, \Omega .
\end{array}
$$

Also note that $U(\vec{z})$ is called an $n$-variable PS, of order $\Omega$. The number of monomials for an $n$-variable PS, of order $\Omega$, is given by

$$
\eta=\frac{(n+\Omega) !}{n ! \Omega !} .
$$

Other symbols most often used for the PSs throughout this paper are $V(\vec{z})$ and $W(\vec{z})$.

A unit PS, of order $\Omega$, is defined as

$$
I(\vec{z})=\sum_{k=0}^{\Omega} i(\vec{k}) \vec{z}^{\vec{k}}=1
$$


i.e.,

$$
i(\vec{k})=1 \quad \text { for } k=0
$$

and

$$
i(\vec{k})=0 \quad \text { for } k>0
$$

\subsubsection{Vector Power Series}

Let $\vec{U}(\vec{z})$ be an $m$-dimensional vector power series (VPS), of $n$ variables and of $\Omega$ order. It is expressed as

$$
\vec{U}(\vec{z})=\sum_{k=0}^{\Omega} \vec{u}(\vec{k}) \vec{z}^{\vec{k}}
$$

Each component of the VPS $\vec{U}(\vec{z})$ is a PS which is given by

$$
U_{i}(\vec{z})=\sum_{k=0}^{\Omega} u_{i}(\vec{k}) \vec{z}^{\vec{k}}, \quad \forall \quad i=1,2, \ldots, m ;
$$

that is, the transposes of $\vec{U}(\vec{z})$ and $\vec{u}(\vec{k})$ are given, respectively, by

$$
\vec{U}^{T}(\vec{z})=\left(U_{1}(\bar{z}), U_{2}(\bar{z}), \ldots, U_{m}(\vec{z})\right)
$$

and

$$
\vec{u}^{T}(\vec{k})=\left(u_{1}(\vec{k}), u_{2}(\vec{k}), \ldots, u_{m}(\vec{k})\right) .
$$

One can consider $\vec{U}(\vec{z})$ as a Taylor map in accelerator physics. Other symbols most often used for VPSs throughout this paper are $\vec{V}(\vec{z})$ and $\vec{W}(\vec{z})$. Note that a homogeneous VPS of degree $i$ is denoted as $\vec{U}_{i}(\vec{z})$ [or $\vec{V}_{i}(\vec{z})$, etc.]. However, a homogeneous PS (or polynomial) of degree $i$ is not denoted as $U_{i}(\vec{z})$ since $U_{i}(\vec{z})$ is reserved as a component of the VPS $\vec{U}(\vec{z})$. Usually, a homogeneous polynomial of degree $i$ is denoted as $f_{i}(\vec{z})$ or $g_{i}(\vec{z})$.

A unit $n$-dimensional, $n$-variable VPS of order $\Omega$ is defined as

$$
\vec{I}(\vec{z})=\sum_{k=0}^{\Omega} \vec{i}(\vec{k}) \vec{z}^{\vec{k}}=\vec{z} .
$$

Its transpose is given by

$$
\vec{I}^{T}(\vec{z})=\vec{z}^{T}=\left(z_{1}, z_{2}, \ldots, z_{n}\right)
$$




\subsubsection{Numerical Implementations}

Since a PS or a VPS is determined once its coefficients are given, $u(\vec{k}), i(\vec{k})$, $\vec{u}(\vec{k})$, and $\vec{i}(\vec{k})$ are numerically used for representing $U(\vec{z}), I(\vec{z}), \vec{U}(\vec{z})$, and $\vec{I}(\vec{z})$, respectively; that is, only the coefficients are stored and manipulated for PS or VPS in a computer. However, note that for the sake of efficient management of computer memories a PS $u(\vec{k})$ must be handled as a one-dimensional array and an $m$-dimensional VPS $\vec{u}(\vec{k})$ as an $m$-dimensional array; that is, a data structure has to be set up for the one-to-one correspondence between a group of sequential integers and the group of all the indexes $\vec{k}$. These one-to-one correspondences are usually represented by well-structured integer pointers which are set up and stored before any operations are performed. For example, a three-variable PS of order 3 has $\eta=(3+3) ! /(3 ! 3 !)=20$ monomials. Two examples of the one-to-one correspondence for this case are given in Table 3.1.

Table 3.1. Examples of 1-D Representations of a Vector Index $\vec{k}$.

\begin{tabular}{ccccccc}
\hline \multicolumn{3}{c}{ Example 1} & & \multicolumn{3}{c}{ Example 2 } \\
\cline { 1 - 2 } \cline { 5 - 6 } sequence & order & $\vec{k}$ & & sequence & order & $\vec{k}$ \\
\hline 1 & 0 & $(0,0,0)$ & & 1 & 0 & $(0,0,0)$ \\
2 & 1 & $(1,0,0)$ & & 1 & $(1,0,0)$ \\
3 & 1 & $(0,1,0)$ & & 3 & 2 & $(2,0,0)$ \\
4 & 1 & $(0,0,1)$ & & 4 & 3 & $(3,0,0)$ \\
5 & 2 & $(2,0,0)$ & & 5 & 1 & $(0,1,0)$ \\
6 & 2 & $(1,1,0)$ & & 6 & 2 & $(1,1,0)$ \\
7 & 2 & $(1,0,1)$ & & 3 & $(2,1,0)$ \\
8 & 2 & $(0,2,0)$ & & 2 & 2 & $(0,2,0)$ \\
9 & 2 & $(0,1,1)$ & & 3 & $(1,2,0)$ \\
10 & 2 & $(0,0,2)$ & & 10 & 3 & $(0,3,0)$ \\
11 & 3 & $(3,0,0)$ & & 11 & 1 & $(0,0,1)$ \\
12 & 3 & $(2,1,0)$ & & 12 & 2 & $(1,0,1)$ \\
13 & 3 & $(2,0,1)$ & & 13 & 3 & $(2,0,1)$ \\
14 & 3 & $(1,2,0)$ & & 14 & 2 & $(0,1,1)$ \\
15 & 3 & $(1,1,1)$ & & 15 & 3 & $(1,1,1)$ \\
16 & 3 & $(1,0,2)$ & & 16 & 3 & $(0,2,1)$ \\
17 & 3 & $(0,3,0)$ & & 17 & 2 & $(0,0,2)$ \\
18 & 3 & $(0,2,1)$ & & 18 & 3 & $(1,0,2)$ \\
19 & 3 & $(0,1,2)$ & & 19 & 3 & $(0,1,2)$ \\
20 & 3 & $(0,0,3)$ & & 20 & 3 & $(0,0,3)$ \\
\hline
\end{tabular}




\subsubsection{Symplectic Identity}

The symplectic identity is given by

$$
S=\left[\begin{array}{cc}
0 & -1 \\
1 & 0
\end{array}\right] \quad \text { for } 2 \times 2 \text { matrices }
$$

The $2 m \times 2 m$ symplectic identity is a block diagonal matrix composed of $m 2 \times 2$ symplectic identities. For example, a $4 \times 4$ symplectic identity would be given by

$$
S=\left[\begin{array}{cc}
S & 0_{2 \times 2} \\
0_{2 \times 2} & S
\end{array}\right], \text { where } 0_{2 \times 2}=\left(\begin{array}{cc}
0 & 0 \\
0 & 0
\end{array}\right)
$$

As a convention, $S$ is used to represent the symplectic identity regardless of its dimension.

\subsubsection{Lie Operators}

Let $f(\vec{z})$ be a function of the phase-space variables represented by $\vec{z}$. Each $f(\vec{z})$ is associated with a Lie operator denoted by the symbol : $f(\vec{z}):$, which is a differential operator defined by

$$
: f(\vec{z}): \equiv[f(\vec{z}),]=-\left(\frac{\partial f(\vec{z})}{\partial \vec{z}}\right)^{T} S \frac{\partial}{\partial \vec{z}}
$$

where the bracket [,] denotes the Poisson bracket of classical mechanics. For example, let $g(\vec{z})$ be another function of the phase-space variables. One finds

$$
: f(\vec{z}): g(\vec{z})=[f(\vec{z}), g(\vec{z})]=-\left(\frac{\partial f(\vec{z})}{\partial \vec{z}}\right)^{T} S \frac{\partial g(\vec{z})}{\partial \vec{z}} .
$$

Positive powers of a Lie operator take repeated Poisson brackets, while zero power of a Lie operator is the identity operator. For example,

$$
\begin{gathered}
: f(\vec{z}):^{2} g(\vec{z})=[f(\vec{z}),: f(\vec{z}): g(\vec{z})]=[f(\vec{z}),[f(\vec{z}), g(\vec{z})]] \\
: f(\vec{z}):^{n} g(\vec{z})=\left[f(\vec{z}),: f(\vec{z}):^{n-1} g(\vec{z})\right] \\
: f(\vec{z}):^{0} g(\vec{z})=g(\vec{z}) .
\end{gathered}
$$


A Lie operator : $f(\vec{z})$ : operating on a VPS $\vec{U}(\vec{z})$ gives a VPS $\vec{W}(\vec{z})$. Each component is given by

$$
W_{i}(\vec{z})=: f(\vec{z}): U_{i}(\vec{z})=\left[f(\vec{z}), U_{i}(\vec{z})\right] .
$$

Thus

$$
: f(\vec{z}): \vec{U}(\vec{z})=[f(\vec{z}), \vec{U}(\vec{z})]=-\left(\frac{\partial f(\vec{z})}{\partial \vec{z}}\right)^{T} S \frac{\partial}{\partial \vec{z}} \vec{U}(\vec{z}) .
$$

A Lie transformation associated with a Lie operator : $f(\vec{z}):$ is denoted as $\exp (: f(\vec{z}):)$ and is given by taking the power series expansion of the exponential function; that is,

$$
\exp (: f(\vec{z}):)=\sum_{m=0}^{\infty} \frac{1}{m !}: f(\vec{z}):^{m}
$$

Given a homogeneous polynomial $f_{i}(\vec{z})$ of order $i$, the associated Lie transformation $\exp \left(: f_{i}(\vec{z}):\right)$ is called a homogeneous Lie transformation of degree $i$ throughout this paper.

For a more complete study of Lie algebraic methods, please refer to Dragt et al. ${ }^{12}$

\subsection{Puwer Series Operations}

\subsubsection{Fundamental Power Series Operations}

There are five fundamental operations for the PS that do not rely on other PS operations. These operations have to be performed with the fundamental setup of data structures and the commonly used scalar operations such as scalar addition, scalar subtraction, scalar multiplication, and scalar division. Neat int eger pointers are usually preset so that these operations can be performed efficiently. The efficiency of performing differential algebra depends very much upon the efficiencies of these five fundamental PS operations: additions, subtractions, multiplications, partial integrations, and partial derivatives. The efficiency of PS multiplication is especially crucial to the successful performance of the differential algebra.

Addition and Subtraction: $\quad W(\vec{z})=U(\vec{z}) \pm V(\vec{z})$

$$
\begin{aligned}
& \sum_{k=0}^{\Omega} w(\vec{k}) \vec{z}^{\vec{k}}=\sum_{k=0}^{\Omega} u(\vec{k}) \vec{z}^{\vec{k}} \pm \sum_{k=0}^{\Omega} v(\vec{k}) \vec{z}^{\vec{k}}=\sum_{k=0}^{\Omega}(u(\vec{k}) \pm v(\vec{k})) \vec{z}^{\vec{k}} \\
& \Rightarrow \quad w(\vec{k})=u(\vec{k}) \pm v(\vec{k}) .
\end{aligned}
$$


Multiplication: $\quad W(\bar{z})=U(\vec{z}) * V(\vec{z})$

$$
\begin{aligned}
\sum_{j=0}^{\Omega} w(\vec{j}) \vec{z}^{j}+\sigma(\Omega+1) & =\left(\sum_{k=0}^{\Omega} u(\vec{k}) \vec{z}^{\vec{k}}\right) *\left(\sum_{h=0}^{\Omega} v(\vec{h}) \vec{z}^{\vec{h}}\right) \\
& =\sum_{j=0}^{\Omega}\left(\sum_{k=0}^{j}(u(\vec{k}) * v(\vec{j}-\vec{k})) \vec{z}^{j}+\sigma(\Omega+1)\right. \\
\Rightarrow \quad w(\vec{j}) & =\sum_{k=0}^{j} u(\vec{k}) * v(\vec{j}-\vec{k}) .
\end{aligned}
$$

Note that " $\sigma(\Omega+1)$ " represents those higher-order terms truncated.

Partial integration: $\quad W(\vec{z})=\int U(\vec{z}) d z_{i}$, where $i=1,2, \ldots$, or $n$

$$
\begin{aligned}
& \sum_{j=1}^{n+1} w(\vec{j}) \vec{z}^{\vec{j}}:=\int \sum_{k=0}^{\Omega} u(\vec{k}) \vec{z}^{\vec{k}} d z_{i}=\sum_{k=0}^{\Omega} \frac{u(\vec{k}) \vec{z}^{\vec{k}+\overrightarrow{1}_{i}}}{k_{i}+1}=\sum_{j=1}^{\Omega+1}\left(\frac{1}{j_{i}}\right) * u\left(\vec{j}-\overrightarrow{1}_{i}\right) \vec{z}^{\vec{j}} \\
& \Rightarrow \quad w(\vec{j})=\left(\frac{1}{j_{i}}\right) * u\left(\vec{j}-\overrightarrow{1}_{i}\right) \text { for } j_{i}>0 .
\end{aligned}
$$

Partial derivative: $\quad W(\vec{z})=\left(\partial / \partial z_{i}\right) U(\vec{z})$, where $i=1,2, \ldots$, or $n$

$$
\begin{aligned}
& \sum_{j=0}^{\Omega} w(\vec{j}) \vec{z}^{\vec{j}}=\frac{\partial}{\partial z_{i}} \sum_{k=1}^{\Omega+1} u(\vec{k}) \vec{z}^{\vec{k}}=\sum_{k=1}^{\Omega+1} k_{i} * u(\vec{k}) \vec{z}^{\vec{k}-\overrightarrow{1}_{i}}=\sum_{j=0}^{\Omega}\left(j_{i}+1\right) * u\left(\vec{j}+\overrightarrow{1}_{i}\right) \vec{z}^{j} \\
& \Rightarrow \quad w(\vec{j})=\left(j_{i}+1\right) * u\left(\vec{j}+\overrightarrow{1}_{i}\right) .
\end{aligned}
$$

These are the fundamental operations for the PS. The objective is to obtain all the coefficients $w(\vec{j})$ (or $w(\vec{k}))$ through computation for the resulting PS $W(\vec{z})$.

\subsubsection{Basic Power Series Operations}

Once the data-structure pointers are set up and the five fundamental PS operations are established computationally, other PS operations can always be performed by using one or more of the five fundamental and other subsequently established PS operations. The purpose, of course, is to obtain all the coefficients $w(\vec{j})$ of the resulting PS $W(\vec{z})$. The following basic PS operations have been established in the numerical library "Zlib." 


$\begin{array}{ll}\text { Square: } & W(\vec{z})=U^{2}(\vec{z}), \\ \text { Inversion: } & W(\vec{z})=1 / U(\vec{z}), \\ \text { Division: } & W(\vec{z})=U(\vec{z}) / V(\vec{z}), \\ \text { Power: } & W(\bar{z})=U^{p}(\vec{z}), \text { where } p \text { is an integer. } \\ \text { Square root: } & W(\vec{z})=\operatorname{sqrt}(U(\vec{z})), \\ \text { Exponentiation: } & W(\vec{z})=\exp (U(\vec{z})), \\ \text { Logarithrs: } & W(\vec{z})=\ln (U(\vec{z})), \\ \text { Trigonometry: } & W(\vec{z})=\sin (U(\vec{z})) \text {, or } W(\vec{z})=\cos (U(\vec{z})), \\ \text { Poisson bracket: } & W(\vec{z})=[U(\vec{z}), V(\vec{z})] .\end{array}$

In obtaining these basic PS operations, power series expansion of the PS is often necessary before using the fundamental PS operations. For example, in order to obtain the PS $W(\vec{z})=\sin (U(\vec{z}))$, one first obtains and stores the coefficients of the power series expansion of the sine function and then applies the fundamental and other more basic PS operations to obtain the coefficients $w(\vec{j})$ of the PS $W(\vec{z})$.

\subsection{Vector Power Series Operations}

With the preset data-structure integer pointers and the fundamental and basic PS operations ready, $\vec{w}(\vec{j})$, the coefficients of the resulting VPS $\vec{W}(\vec{z})$ can be obtained for the following basic VPS operations.

\subsubsection{Concatenation}

Concatenation: $\quad \vec{W}(\vec{z})=\vec{V}(\vec{U}(\vec{z}))$,

where, in the usual case, $\vec{U}$ is an $n$-dimensional, $n$-variable VPS, $\vec{V}$ and $\vec{W}$ are $m$-dimensional, $n$-variable VPS, and $m$ and $n$ may or may not be equal.

\subsubsection{Inversion}

Inversion: $\quad \vec{W}(\vec{z})=\vec{U}^{-1}(\vec{z})$.

Given an $n$-dimensional, $n$-variable $\vec{U}(\vec{z})$, an $n$-dimensional, $n$-variable $\vec{U}^{-1}(\vec{z})$ can be obtained such that

$$
\vec{U}^{-1}(\vec{U}(\vec{z}))=\vec{U}\left(\vec{U}^{-1}(\vec{z})\right)=\vec{I}(\vec{z}) .
$$

These basic VPS operations have been implemented in the numerical library "Zlib." 


\subsection{Global and Local Operators}

To prepare for the discussion of the Dragt-Finn factorization, the kick factorization, and the normal form in the next sections, let us demonstrate the global forms and the local forms of transformations with the following example.

Consider two symplectic matrices given by

$$
R_{1}=\left(\begin{array}{cc}
\cos \mu_{1} & \sin \mu_{1} \\
-\sin \mu_{1} & \cos \mu_{1}
\end{array}\right)
$$

and

$$
M=\left(\begin{array}{cc}
\cos \mu \cdot \alpha \sin \mu & \beta \sin \mu \\
-\gamma \sin \mu & \cos \mu-\alpha \sin \mu
\end{array}\right) \quad \text { with } \quad \gamma=\frac{1+\alpha^{2}}{\beta},
$$

which transform the initial phase-space coordinates $\vec{z}^{T}=(x, p)$ to the final phase-space coordinates $\vec{z}_{f}^{T}=\left(x_{f}, p_{f}\right)$ by consecutive operation of the two matrices on the coordinates as follows:

$$
\begin{aligned}
\left(\begin{array}{l}
x_{f} \\
p_{f}
\end{array}\right)=\vec{z}_{f}=M R_{1} \vec{z}= & \left(\begin{array}{cc}
\cos \mu+\alpha \sin \mu & \beta \sin \mu \\
-\gamma \sin \mu & \cos \mu-\alpha \sin \mu
\end{array}\right) \\
& \left(\begin{array}{cc}
\cos \mu_{1} & \sin \mu_{1} \\
-\sin \mu_{1} & \cos \mu_{1}
\end{array}\right)\left(\begin{array}{l}
x \\
p
\end{array}\right) .
\end{aligned}
$$

The definition of these two consecutive transformations is actually a two-step transformation. The first step is to transform the initial coordinate $\vec{z}^{T}=(x, p)$ to a medium step coordinate $\vec{z}_{m}^{T}=\left(x_{m}, p_{m}\right)$ given by

$$
\left(\begin{array}{c}
x_{m} \\
p_{m}
\end{array}\right)=\vec{z}_{m}=R_{1} \vec{z}=\left(\begin{array}{cc}
\cos \mu_{1} & \sin \mu_{1} \\
-\sin \mu_{1} & \cos \mu_{1}
\end{array}\right)\left(\begin{array}{l}
x \\
p
\end{array}\right)=\left(\begin{array}{c}
\left(\cos \mu_{1}\right) x+\left(\sin \mu_{1}\right) p \\
-\left(\sin \mu_{1}\right) x+\left(\cos \mu_{1}\right) p
\end{array}\right) \text {. }
$$

The second step is to transform the medium step coordinate $\vec{z}_{m}^{T}=\left(x_{m}, p_{m}\right)$ to the final coordinate $\vec{z}_{f}^{T}=\left(x_{f}, p_{f}\right)$ given by

$$
\begin{aligned}
\left(\begin{array}{l}
x_{f} \\
p_{f}
\end{array}\right)= & \vec{z}_{f}=M \vec{z}_{m}=\left(\begin{array}{cc}
\cos \mu+\alpha \sin \mu & \beta \sin \mu \\
-\gamma \sin \mu & \cos \mu-\alpha \sin \mu
\end{array}\right)\left(\begin{array}{l}
x_{m} \\
p_{m}
\end{array}\right) \\
= & \left(\begin{array}{c}
(\cos \mu+\alpha \sin \mu) x_{m}+(\beta \sin \mu) p_{m} \\
-(\gamma \sin \mu) x_{m}+(\cos \mu-\alpha \sin \mu) p_{m}
\end{array}\right) \\
= & \left(\begin{array}{c}
\left(\cos \mu \cos \mu_{1}+\alpha \sin \mu \cos \mu_{1}-\beta \sin \mu \sin \mu_{1}\right) x \\
+\left(\cos \mu \sin \mu_{1}+\alpha \sin \mu \sin \mu_{1}+\beta \sin \mu \cos \mu_{1}\right) p \\
-\left(\gamma \sin \mu \cos \mu_{1}+\cos \mu \sin \mu_{1}-\alpha \sin \mu \sin \mu_{1}\right) x \\
-\left(\gamma \sin \mu \sin \mu_{1}-\cos \mu \cos \mu_{1}+\alpha \sin \mu \cos \mu_{1}\right) p
\end{array}\right) .
\end{aligned}
$$


Clearly, matrices are local operators. On the other hand, let us consider two Lie transformations associated with the two symplectic matrices $R_{1}$ and $M$ given by

$$
\mathcal{R}_{1}(\vec{z})=e^{:-\mu_{1}\left(x^{2}+p^{2}\right) / 2}
$$

and

$$
\mathcal{M}(\vec{z})=e^{:-\mu\left(\gamma x^{2}+\beta p^{2}+2 \alpha x p\right) / 2:} .
$$

By applying the Lie algebraic rules given in Section 3.1.6, we see that the $\vec{z}_{f}$ given by Eq. (3.2) can be obtained by the operation of the two associated Lie transformations $\mathcal{R}_{1}(\vec{z}), \mathcal{M}(\vec{z})$ but in the reverse order; that is,

$$
\vec{z}_{f}=\mathcal{R}_{1}(\vec{z}) \mathcal{M}(\vec{z}) \vec{z} .
$$

Both of the Lie transformations operate on the global (initial) coordinates $\vec{z}$ as they are both functional forms of $\vec{z} \cdot \mathcal{R}_{1}(\vec{z})$ and $\mathcal{M}(\vec{z})$ are called the global forms of $R_{1}$ and $M$, respectively.

Based on this example, we make the following conclusion without further demonstration or proof.

Consider a series of symplectic local operators, $L_{1}, L_{2}, \ldots, L_{m}$, not necessarily Courant-Snyder matrices. Associated with these local operators are their corresponding global operators of functional forms of the global coordinates (initial phase-space coordinates) $\vec{z}$ given by $\mathcal{L}_{1}(\vec{z}), \mathcal{L}_{2}(\vec{z}), \ldots, \mathcal{L}_{m}(\vec{z})$, respectively. To obtain the same transformation results through their consecutive operations, the operational sequence for the global forms must be in reverse order of the local operational sequence.

\subsection{Theorems for Symplectic Maps and Lie Operators}

Also needed for the discussion in the next sections are the following theorems for symplectic maps and Lie operators. They are listed without proof. However, the reader is encouraged to convince himself with a few simple examples that these theorems are indeed correct.

Theorem 1: Let $m(\vec{z})$ be a symplectic map. Then its inverse $m^{-1}(\vec{z})$ is also a symplectic map.

Theorem 2: Let $m_{1}(\vec{z}), m_{2}(\vec{z})$ be symplectic maps. Then the concatenated map $m(\vec{z})=m_{1}(\bar{z}) m_{2}(\vec{z})$ is also a symplectic map.

Theorem 3: Let $m(\vec{z})$ be a symplectic map and $f(\vec{z})$ be a function of the phase-space variables represented by $\vec{z}$. Then

$$
m(\vec{z}) f(\vec{z})=f(m(\vec{z}) \vec{z}) .
$$


For example, let us consider $\vec{z}^{T}=(x, p)$ and the rotational matrix $R_{1}$ and its associated global form $\mathcal{R}_{1}(\vec{z})$, given in the last section. Note that a rotational matrix is symplectic. It can be easily checked that

$$
\mathcal{R}_{1}(\vec{z}) f(\vec{z})=f\left(\mathcal{R}_{1}(\vec{z}) \vec{z}\right)=f\left(R_{1} \vec{z}\right) .
$$

Theorem 4: Let $f(\vec{z}), g(\vec{z})$ be functions of the phase-space variables represented by $\vec{z}$. Then

$$
\{: f(\vec{z}):,: g(\vec{z}):\} \equiv: f(\vec{z}):: g(\vec{z}):-: g(\vec{z}):: f(\vec{z}):=:[f(\vec{z}), g(\vec{z})]: .
$$

Theorem 5: Let $m(\vec{z})$ be a symplectic map and $f(\vec{z})$ be a function of the phase-space variables represented by $\vec{z}$. Then

$$
m(\vec{z}) e^{: f(\vec{z}):} m^{-1}(\vec{z})=e^{: m(\vec{z}) f(\vec{z}):}=e^{: f(m(\vec{z}) \vec{z}):} .
$$

For example:

$$
e^{: g(\vec{z}):} e^{: f(\vec{z}):} e^{-: g(\bar{z}):}=\exp \left(: e^{: g(\vec{z}):} f(\vec{z}):\right) .
$$

Theorem 6: Let $e^{: h(\bar{z}):}=e^{: f(\bar{z}):} e^{: g(\bar{z}):}$. Then

$h(\vec{z})=f(\vec{z})+g(\vec{z})+\frac{1}{2}[f(\vec{z}), g(\bar{z})]+\frac{1}{12}[f(\vec{z}),[f(\vec{z}), g(\bar{z})]]+\frac{1}{12}[g(\vec{z}),[g(\vec{z}), f(\vec{z})]]+\ldots$

This is the Campbell-Baker-Hausdorff (CBH) theorem. ${ }^{13-15}$

\subsection{Dragt-Finn Factorization}

As we shall study in the next chapter, a map representing a one-turn storage ring (or a beam line) can be formulated as follows:

$$
\vec{z}^{\prime}=m \vec{z}=\vec{U}(\vec{z})+\sigma(\Omega+1)
$$

where $\vec{U}(\vec{z})$ is a VPS of order $\Omega$, and $\sigma(\Omega+1)$ represents all the truncated high$\operatorname{order}(>\Omega)$ terms. This is usually called the Taylor map. Due to symplecticity of the system, the map can also be formulated as a series of Lie transformations, the so called Dragt-Finn factorization map, given by ${ }^{4}$

$$
\vec{z}^{\prime}=m \vec{z}=\prod_{i=1}^{\Omega} e^{: f_{i}(\vec{z}):} \vec{z}+\sigma(\Omega+1),
$$

where $f_{i}(\vec{z})$ is a homogeneous polynomial of order $i$. 
A Dragt-Finn factorization map is always symplectic, regardless of the order to which the series of Lie transformation is extended. A Taylor map, on the other hand, is generally not symplectic becciuse of truncation of the high orders, but it still preserves order-by-order symplectic property of the system. A Taylor map representing a symplectic system can always be converted to a Dragt-Finn factorization map with an accuracy up to the truncated order, $\Omega$, of the Taylor map. The procedures are described below.

For simplicity without losing generality, let us consider a one-turn onmomentum transverse map of a storage ring and follow the phase-space coordinate convention as given by Eq. (3.1). We shall further consider that the coordinates $x, y$ and their conjugate momenta $p_{x}, p_{y}$ are deviations of particle phase-space trajectories with respect to the closed orbit. Thus $m \overrightarrow{0}=\overrightarrow{0}$, and Eq. (3.3) can be rewritten as

$$
m \vec{z}=\vec{U}(\vec{z})+\sigma(\Omega+1)=\vec{U}_{1}(\vec{z})+\sum_{i=2}^{\Omega} \vec{U}_{i}(\vec{z})+\sigma(\Omega+1),
$$

where $\vec{U}_{1}(\vec{z})$ is the linear map which, alone, is symplectic. The linear map can be re-formed as

$$
\vec{U}_{1}(\vec{z})=\mathcal{M}(\vec{z}) \vec{z}=M \vec{z},
$$

where $M$ is a Courant-Snyder matrix and $\mathcal{M}(\vec{z})$ is its global form. The symplecticity of the linear map leads to

$$
M^{T} S M=S .
$$

Thus, one can always find a canonical generation matrix $A$ constructed by the eigenvectors of $M^{T}$ such that it transforms $M$ into a rotation $R_{;}^{16}$ that is,

$$
R=\left(\begin{array}{cccc}
\cos \mu_{x} & \sin \mu_{x} & 0 & 0 \\
-\sin \mu_{x} & \cos \mu_{x} & 0 & 0 \\
0 & 0 & \cos \mu_{y} & \sin \mu_{y} \\
0 & 0 & -\sin \mu_{y} & \cos \mu_{y}
\end{array}\right)=A^{-1} M A,
$$

where $\mu_{x}=2 \pi \nu_{x}$ and $\mu_{y}=2 \pi \nu_{y}$ are the fractional betatron tunes discussed in Chapter 2. Denoting the global forms of the symplectic matrices $R$ and $A$ as $\mathcal{R}(\vec{z})$ and $\mathcal{A}(\vec{z})$, respectively, the global form for Eq. (3.6) is given by

$$
\mathcal{R}(\vec{z})=\mathcal{A}(\vec{z}) \mathcal{M}(\vec{z}) \mathcal{A}^{-1}(\vec{z}) .
$$

Now, let us first make a similarity transformation of the map $m$ as follows:

$$
{ }_{1} m=\mathcal{A}(\vec{z}) m \mathcal{A}^{-1}(\vec{z}) .
$$


Using Eq. (3.5) and applying differential algebra for the concatenation of VPSs, one obtains

$$
{ }_{1} m \vec{z}=\mathcal{A}(\vec{z}) m \mathcal{A}^{-1}(\vec{z}) \vec{z}=\mathcal{R}(\vec{z}) \vec{z}+\sum_{i=2}^{\Omega}{ }_{1} \vec{U}_{i}(\vec{z})+\sigma(\Omega+1) .
$$

Making another transformation by operating Eq. (3.7) wich $\mathcal{R}^{-1}(\vec{z})$, one obtains

$$
{ }_{2} m \vec{z}=\mathcal{R}^{-1}(\vec{z}){ }_{1} m \vec{z}=\vec{z}+{ }_{2} \vec{U}_{2}(\vec{z})+\sum_{i=3}^{\Omega}{ }_{2} \vec{U}_{i}(\vec{z})+\sigma(\Omega+1) .
$$

The next step is to find a homogeneous Lie transformation of degree 3, $\exp \left(: f_{3}(\vec{z}):\right)$, such that

$$
e^{: f_{3}(\vec{z}):} \vec{z}=\vec{z}+{ }_{2} \vec{U}_{2}(\vec{z})+\sigma(3)=\vec{z}+{ }_{2} \vec{U}_{2}(\vec{z})+\sum_{i=3}^{\infty}{ }_{2} \vec{V}_{i}(\vec{z}) .
$$

Symplecticity of the system guarantees the existence of such a homogeneous Lie transformation; that is, there exists a homogeneous polynomial $f_{3}(\vec{z})$ of order 3 such that

$$
: f_{3}(\vec{z}): \vec{z}=\left[f_{3}(\vec{z}), \vec{z}\right]=S \frac{\partial f_{3}(\vec{z})}{\partial \vec{z}}={ }_{2} \vec{U}_{2}(\vec{z}) .
$$

The solution for $f_{3}(\vec{z})$ is unique since ${ }_{2} \vec{U}_{2}(\vec{z})$ is conservative; that is, $\nabla \times$ $\left(S_{2} \vec{U}_{2}(\vec{z})\right)=0$, due to the symplectic nature of the system. It is given by

$$
f_{3}(\vec{z})=-\vec{z}^{T} S_{2} \vec{U}_{2}(\vec{z}) / 3
$$

Subtracting Eq. (3.9) from Eq. (3.8), one obtains

$$
{ }_{2} m \vec{z}=e^{: f_{3}(\vec{z}): \vec{z}}+\sum_{i=3}^{\Omega}{ }_{2} \vec{U}_{i}^{\prime}(\vec{z})+\sigma(\Omega+1),
$$

where

$$
{ }_{2} \vec{U}_{i}^{\prime}(\vec{z})={ }_{2} \vec{U}_{i}(\vec{z})-{ }_{2} \vec{V}_{i}(\vec{z}) .
$$

Transforming Eq. (3.10) as follows:

$$
{ }_{3} m \vec{z}=e^{-: f_{3}(\vec{z}):}{ }_{2} m \vec{z}=\vec{z}+{ }_{3} \vec{U}_{3}(\vec{z})+\sum_{i=4}^{\Omega}{ }_{3} \vec{U}_{i}(\vec{z})+\sigma(\Omega+1),
$$

one obtains an equation similar to Eq. (3.8). Thus, following the same procedures iteratively, one finally obtains the rest of the homogeneous Lie generators 
of higher degrees given by

$$
f_{k}(\vec{z})=-\vec{z}^{T} S_{k-1} \vec{U}_{k-1}(\vec{z}) / k \quad \forall \quad k=4,5, \ldots, \Omega+1,
$$

and the associated transformed map given by

$$
{ }_{k} m \vec{z}=e^{-: f_{k}(\vec{z}):}{ }_{k-1} m \vec{z}=\vec{z}+{ }_{k} \vec{U}_{k}(\vec{z})+\sum_{i=K+1}^{\Omega}{ }_{k} \vec{U}_{i}(\vec{z})+\sigma(\Omega+1)
$$

For $k=\Omega+1, \mathrm{Eq}$. (3.12) yields

$$
\Omega+1 m \vec{z}=e^{-: f_{\Omega+1}(\vec{z}):} \Omega_{\Omega} m \vec{z}=\vec{z}+\sigma(\Omega+1) \simeq I \vec{z} .
$$

Therefore, neglecting terms with orders higher than $\Omega$, one obtains

$$
m=\mathcal{A}^{-1}(\vec{z}) m_{f} \mathcal{A}(\vec{z}),
$$

where

$$
m_{f}=\mathcal{R}(\vec{z}) e^{: f_{3}(\vec{z}):} e^{: f_{4}(\bar{z}):} \ldots e^{: f_{\Omega+1}(\bar{z}):} .
$$

This is the Dragt-Finn factorization map. One can also convert a Dragt-Finn factorization map into a Taylor map by using differential algebra for the concatenation of VPSs.

\subsection{Kick Factorizations}

Although the Dragt-Finn factorization map given by Eq. (3.14) is symplectic, evaluation of the Lie transformations usually requires truncated Taylor expansions. Once the Taylor expansions are truncated, the map is not exactly symplectic anymore.

Irwin demonstrated a method of factorizing the Dragt-Finn factorization map into a series of rotations and kicks so that the map can be evaluated rapidly and symplectically with an accuracy up to order $\Omega$ in terms of Taylor expansion. ${ }^{17}$ Later, Dragt pointed out that the total number of kicks could be reduced by nearly half of Irwin's by choosing a more general unitary group instead of a rotational group. ${ }^{18}$ However, Dragt's method is more complicated. The basic idea of these kick factorizations is to convert the series of Lie transformations given by Eq. (3.14) into another series of Lie transformations given by

$$
m_{f}=\mathcal{R}(\vec{z}) e^{: g_{1}\left(\vec{\xi}_{1}\right):} e^{: g_{2}\left(\vec{\xi}_{2}\right):} \ldots e^{: g_{k}\left(\vec{\xi}_{k}\right):} \ldots e^{: g_{\Gamma}\left(\vec{\xi}_{\Gamma}\right):}+\sigma(\Omega+1)
$$

where each $g_{k}\left(\vec{\xi}_{k}\right)$ for $k=1,2, \ldots, \Gamma$ is a power series of $\vec{\xi}_{k}$ o.l.y, and $\vec{\xi}_{1}, \vec{\xi}_{2}, \ldots \vec{\xi}_{k}, \ldots \vec{\xi}_{r}$ are the kick factorization bases. Each $\vec{\xi}_{k}$ is a vector representing the canonical conjugate coordinates only. Its corresponding phase-space 
coordinates (both the conjugate coordinates and momenta) are represented by $\vec{z}_{k}$ given, in general, by

$$
\vec{z}_{k}=M_{k} \vec{z}
$$

where each $M_{k}$ for $k=1,2, \ldots, \Gamma$ is a matrix, and $\vec{z}$, as usual, is the vector representing the global phase-space coordinates. Taking the transverse map, for example, each $\vec{z}_{k}$ and each $\vec{\xi}_{k}$ is given, respectively, by

$$
\left(\begin{array}{c}
x_{k} \\
p_{x k} \\
y_{k} \\
p_{y k}
\end{array}\right)=\vec{z}_{k}=M_{k} \vec{z}=M_{k}\left(\begin{array}{c}
x \\
p_{x} \\
y \\
p_{y}
\end{array}\right)
$$

and

$$
\vec{\xi}_{k}=\left(\begin{array}{l}
x_{k} \\
y_{k}
\end{array}\right) .
$$

For convenience, we shall also define

$$
\vec{\xi}=\left(\begin{array}{l}
x \\
y
\end{array}\right) .
$$

The Lie transformations given by Eq. (3.15) are called kick factorizations. Not only are they symplectic, but also they can be evaluated without truncation by further manipulations as follows:

$$
\begin{aligned}
m_{f} & =\mathcal{R} e^{: g_{1}\left(\vec{\xi}_{1}\right):} e^{: g_{2}\left(\vec{\xi}_{2}\right):} \ldots e^{: g_{k}\left(\vec{\xi}_{k}\right):} \ldots e^{: g_{\Gamma}\left(\vec{\xi}_{\Gamma}\right):} \\
& =\mathcal{R} e^{: g_{1}\left(\vec{z}_{1}\right):}: e^{: g_{2}\left(\vec{z}_{2}\right):} \ldots e^{: g_{k}\left(\vec{z}_{k}\right):} \ldots e^{: g_{\Gamma}\left(\vec{z}_{\Gamma}\right):} \\
& =\mathcal{R} e^{: g_{1}\left(\mathcal{M}_{1} \vec{z}\right):} e^{: g_{2}\left(M_{2} \vec{z}\right):} \ldots e^{: g_{k}\left(\mathcal{M}_{k} \vec{z}\right):} \ldots e^{: g_{\Gamma}\left(M_{\Gamma} \vec{z}\right):} \\
& =\mathcal{R} e^{: \mathcal{M}_{1} g_{1}(\vec{z}):} e^{: \mathcal{M}_{2} g_{2}(\vec{z}):} \ldots e^{: \mathcal{M}_{k} g_{k}(\vec{z}):} \ldots e^{: \mathcal{M}_{\Gamma} \cdot g_{\Gamma}(\vec{z}):} \\
& =\mathcal{R} \mathcal{M}_{1} e^{: g_{1}(\vec{z}):} \mathcal{M}_{1}^{-1} \mathcal{M}_{2} e^{: g_{2}(\vec{z}):} \mathcal{M}_{2}^{-1} \ldots \mathcal{M}_{k} e^{: g_{k}(\vec{z}):} \mathcal{M}_{k}^{-1} \ldots \mathcal{M}_{\Gamma} e^{: g_{\Gamma}(\vec{z}):} \mathcal{M}_{\Gamma}^{-1} \\
& =\mathcal{R} \mathcal{M}_{1} e^{: g_{1}(\vec{\xi}):} \mathcal{M}_{1}^{-1} \mathcal{M}_{2} e^{: g_{2}(\vec{\xi}):} \mathcal{M}_{2}^{-1} \ldots \mathcal{M}_{k} e^{: g_{k}(\vec{\xi}):} \mathcal{M}_{k}^{-1} \ldots \mathcal{M}_{\Gamma} e^{: g_{\Gamma}(\vec{\xi}):} \mathcal{M}_{\Gamma}^{-1}
\end{aligned}
$$

where $\mathcal{M}_{k}$ and its inverse $\mathcal{M}_{k}^{-1}$ are the global forms of the matrix $M_{k}$ and its inverse $M_{k}^{-1}$, respectively, for $k=1,2, \ldots, \Gamma$. More precisely, $\mathcal{R}, \mathcal{M}_{k}$, and $\mathcal{M}_{k}^{-1}$ should be written as $\mathcal{R}(\vec{z}), \mathcal{M}_{k}(\vec{z})$, and $\mathcal{M}_{k}^{-1}(\vec{z})$.

Note that in Eq. (3.17) we have let $g_{k}\left(\vec{z}_{k}\right)=g_{k}\left(\vec{\xi}_{k}\right)$ and $g_{k}(\vec{\xi})=g_{k}(\vec{z})$ for $k=1,2, \ldots, \Gamma$ because the coefficients for the monomials with non-zero order in conjugate momenta- $\left(p_{x k}, p_{y k}\right)$ or $\left(p_{x}, p_{y}\right)$, for example-are ail $U$ for the power 
series $g_{k}$. Each of the Lie transformations, $\exp \left(: g_{k}(\vec{\xi}):\right)$ for $k=1,2, \ldots, \Gamma$, is a kick given by

$$
\vec{z}^{\prime}=e^{: g_{k}(\vec{\xi}): \vec{z}}=\vec{z}+\left[g_{k}(\vec{\xi}), \vec{z}\right]
$$

Taking the transverse map, for example, again where $\vec{z}^{T}=\left(x, p_{x}, y, p_{y}\right)$ and $\vec{\xi}^{T}=(x, y)$, we have $\vec{z}^{\prime T}=\left(x^{\prime}, p_{x}^{\prime}, y^{\prime}, p_{y}^{\prime}\right)$, where

$$
\left(\begin{array}{c}
x^{\prime} \\
p_{x}^{\prime} \\
y^{\prime} \\
p_{y}^{\prime}
\end{array}\right)=\vec{z}^{\prime}=e^{: g_{k}(x, y):} \vec{z}=\left(\begin{array}{c}
x \\
p_{x}+\frac{\partial g_{k}(x, y)}{\partial x} \\
y \\
p_{y}+\frac{\partial g_{k}(x, y)}{\partial y}
\end{array}\right) .
$$

Note that evaluation of $m_{f}$ with the kick factorizations given by Eq. (3.15) is accurate to the order $\Omega$ in terms of Taylor expansions. However, the kick factorizations are not unique. They depend on how the factorization bases $\vec{\xi}_{1}, \vec{\xi}_{2}, \ldots \vec{\xi}_{r}$ are chosen. For illustration as to how kick factorizations given by Eq. (3.15) are obtained, we shall consider a transverse map and follow the method proposed by Irwin with slight modification. The reader is encouraged to refer to the method proposed by Dragt, which requires fewer minimum kicks. ${ }^{18}$

\subsubsection{Irwin Kick Factorization Bases}

In Irwin's proposal, the matrices $M_{k}$ for $k=1,2, \ldots \Gamma$ are all rotational matrices which we shall denote as

$$
R_{k}=\left(\begin{array}{cccc}
\cos \theta_{x k} & \sin \theta_{x k} & 0 & 0 \\
-\sin \theta_{x k} & \cos \theta_{x k} & 0 & 0 \\
0 & 0 & \cos \theta_{y k} & \sin \theta_{y k} \\
0 & 0 & -\sin \theta_{y k} & \cos \theta_{y k}
\end{array}\right)
$$

Thus,

$$
\left(\begin{array}{c}
x_{k} \\
p_{x k} \\
y_{k} \\
p_{y k}
\end{array}\right)=\vec{z}_{k}=\left(\begin{array}{c}
x \cos \theta_{x k}+p_{x} \sin \theta_{x k} \\
-x \sin \theta_{x k}+p_{x} \cos \theta_{x k} \\
y \cos \theta_{y k}+p_{y} \sin \theta_{y k} \\
-y \sin \theta_{y k}+p_{y} \cos \theta_{x k}
\end{array}\right) .
$$

What is needed is actually

$$
\vec{\xi}_{k}=\left(\begin{array}{l}
x_{k} \\
y_{k}
\end{array}\right)=\left(\begin{array}{l}
x \cos \theta_{x k}+p_{x} \sin \theta_{x k} \\
y \cos \theta_{y k}+p_{y} \sin \theta_{y k}
\end{array}\right) .
$$


The minimum required number of Irwin factorization bases, $\Gamma$, is given by

$$
\Gamma_{\min }= \begin{cases}(\Omega+3)^{2} / 4 & \text { if } \Omega \text { is an odd number } \\ (\Omega+2)(\Omega+4) / 4 & \text { if } \Omega \text { is an even number. }\end{cases}
$$

Eq. (3.18) will become clear as our discussion continues.

Once the factorization base number $\Gamma\left(\Gamma \geq \Gamma_{\min }\right)$ is decided, the next step is to choose the factorization basis set $\overrightarrow{\vec{\xi}}=\left\{\vec{\xi}_{1}, \vec{\xi}_{2}, \ldots \vec{\xi}_{k}, \ldots \vec{\xi}_{\Gamma}\right\}$; that is, to choose a total of $\Gamma$ pairs of rotational angles $\vec{\theta}_{k}=\left(\theta_{x k}, \theta_{y k}\right)$ for $k=1,2, \ldots, \Gamma$, where $0 \leq \theta_{x k}, \theta_{y k}<\pi$. One way to choose the $\vec{\theta}_{k}$ 's is to use the random number generators to generate the $\Gamma$ pairs of $\vec{\theta}_{k}$ 's with equal probability for each $\theta_{x k}$ and $\theta_{y k}$ to be between 0 and $\pi$. A criterion is set up such that if any two pairs of $\vec{\theta}_{k}$ 's are too close, the $\Gamma$ pairs of $\vec{\theta}_{k}$ 's are re-generated to guarantee a sufficient degree of linear independency among the bases $\vec{\xi}_{k}$ 's.

\subsubsection{Kick Factorization of a Homogeneous Lie Transformation}

Now that the kick factorization basis has been discussed, let us first consider converting a homogeneous Lie transformation $\exp \left(: f_{i}(\vec{z}):\right)$ of order $i$ into kick factorizations.

Let $f_{i}(\vec{z})=\sum_{m+n=i} f_{i}^{m n}(\vec{z})$, where, in general,

$$
f_{i}^{m n}(\vec{z})=\sum_{r=0}^{m} \sum_{s=0}^{n} C_{r s}^{m n} x^{r} p_{x}^{m-r} y^{s} p_{y}^{n-s} .
$$

For each $m+n=i$, re-ordering the two indexes, $r, s$, into one index $j$ given by $j=1+r+(n+1) s, 1 \leq j \leq \Gamma^{m n}=(m+1)(n+1) \leq \Gamma$, and defining $\nu_{j}^{m n} \equiv \nu_{r s}^{m n} \equiv x^{r} p_{x}^{m-r} y^{s} p_{y}^{n-s}$, we have

$$
f_{i}^{m n}(\vec{z})=\sum_{j=1}^{\Gamma^{m n}} c_{j}^{m n} \nu_{j}^{m n}
$$

Now let us choose, from the factorization basis set $\overrightarrow{\vec{\xi}}$, a subset $\overrightarrow{\vec{\xi}}^{m n}$ consisting of $\Gamma^{m n}$ bases that are most linear-independent of one another. Let us re-order the $\Gamma^{m n}$ bases in the subset $\overline{\vec{\xi}}^{m n}$ and denote the subset as

$$
\overline{\vec{\xi}}^{m n}=\left\{\vec{\xi}_{1}^{m n}, \vec{\xi}_{2}^{m n}, \ldots, \vec{\xi}_{l}^{m n}, \ldots \vec{\xi}_{\Gamma^{m n}}^{m n}\right\}
$$

where each

$$
\vec{\xi}_{l}^{m n}=\left(\begin{array}{l}
x_{m n, l} \\
y_{m n, l}
\end{array}\right)
$$


for $l=1,2, \ldots \Gamma^{m n}$ also belongs to the factorization basis set $\overrightarrow{\vec{\xi}}$; that is, $\vec{\xi}_{l}^{m n} \in \overrightarrow{\vec{\xi}}$.

Now consider $u_{l}^{m n}=x_{m n, l}^{m} y_{m n, l}^{n}$ for $l=1,2, \ldots \Gamma^{m n}$. Each $u_{l}^{m n}$ can be expanded as follows:

$$
u_{l}^{m n}=\sum_{j=1}^{\Gamma^{m n}} a_{l j}^{m n} \nu_{j}^{m n}
$$

Letting the vectors $\vec{u}_{m n}, \vec{\nu}_{m n}$, and $\vec{c}_{m n}$ be defined such that

$$
\begin{aligned}
& \vec{u}_{m n}^{T}=\left(u_{1}^{m n}, u_{2}^{m n}, \ldots u_{l}^{m n}, \ldots u_{\Gamma^{m n}}^{m n}\right), \\
& \vec{\nu}_{m n}^{T}=\left(\nu_{1}^{m n}, \nu_{2}^{m n}, \ldots \nu_{j}^{m n}, \ldots \nu_{\Gamma^{m n}}^{m n}\right), \\
& \vec{c}_{m n}^{T}=\left(c_{1}^{m n}, c_{2}^{m n}, \ldots c_{j}^{m n}, \ldots c_{\Gamma^{m n}}^{m n}\right),
\end{aligned}
$$

then Eqs. (3.19) and (3.20) can be re-formed and given by

$$
\begin{aligned}
f_{i}^{m n}(\vec{z}) & =\vec{c}_{m n}^{T} \vec{\nu}_{m n}, \\
\vec{u}_{m n} & =M_{m n} \vec{\nu}_{m n},
\end{aligned}
$$

where $M_{m n}$ is a $\Gamma^{m n} \times \Gamma^{m n}$ matrix. Its components are $a_{l j}^{m n}$ for $l=1,2, \ldots \Gamma^{m n}$ and $j=1,2, \ldots \Gamma^{m n}$.

Eq. (3.22) can be inverted such that

$$
\vec{\nu}_{m n}=M_{m n}^{-1} \vec{u}_{m n} \text {. }
$$

Substituting Eq. (3.23) into Eq. (3.21), we obtain

$$
f_{i}^{m n}(\vec{z})=\vec{c}_{m n}^{T} M_{m n}^{-1} \vec{u}_{m n}=\vec{c}_{m n}^{T} \vec{u}_{m n}=\sum_{l=!}^{\Gamma^{m n}} c_{l}^{\prime m n} u_{l}^{m n}=\sum_{l=1}^{\Gamma^{m n}} c_{l}^{\prime m n} x_{m n, l}^{m} y_{m n, l}^{n},
$$

where

$$
\vec{c}_{m n}^{T T}=\left(c_{1}^{\prime m n}, c_{2}^{\prime m n}, \ldots, c_{l}^{\prime m n}, \ldots c_{\Gamma^{m n}}^{\prime m n}\right)=\left(M_{m n}^{-1 T} \vec{c}_{m n}\right)^{T}
$$

Since

$$
\vec{\xi}_{l}^{m n}=\left(\begin{array}{c}
x_{m n, l} \\
y_{m n, l}
\end{array}\right) \in \overrightarrow{\vec{\xi}}
$$

for all $l=1,2, \ldots, \Gamma^{m n}$, in general, we have

$$
\begin{aligned}
f_{i}(\vec{z})=\sum_{m+n=i} f_{i}^{m n}(\vec{z}) & =\sum_{m+n=i} \sum_{l=1}^{\Gamma^{m n}} c_{l}^{\prime m n} x_{m n, l}^{m} y_{m n, l}^{n}=\sum_{k=1}^{\Gamma} \sum_{m+n=i} d_{k}^{m n} x_{k}^{m} y_{k}^{n} \\
& =\sum_{k=1}^{\Gamma} g_{i, k}\left(x_{k}, y_{k}\right),
\end{aligned}
$$

where each $g_{i, k}\left(x_{k}, y_{k}\right)$ for $k=1,2, \ldots, \Gamma$, is a homogeneous polynomial of order $i$. Finally, the homogeneous Lie transformation $\exp \left(: f_{i}(\vec{z}):\right)$ can be 
kick-factorized such that

$$
e^{: f_{i}(\vec{z}): \vec{z}}=m_{g, i} \vec{z}+\sigma(i)
$$

where

$$
m_{g, i} \prod_{k=1}^{\Gamma} e^{: g_{i, k}\left(x_{k}, y_{k}\right):} .
$$

The kick factorization can also be Dragt-Finn factorized as follows:

$$
m_{g, i}=\prod_{k=1}^{\Gamma} e^{: g_{i, k}\left(x_{k}, y_{k}\right)}=e^{: f_{i}(\bar{z}):} e^{: h_{i+1}(\bar{z}):} e^{: h_{i+2}(\bar{z}):} \ldots,
$$

where $h_{i+1}, h_{i+2}, \ldots$ are homogeneous polynomials of degree $i+1, i+2, \ldots$. It is now clear that the required number of bases in the subset $\overrightarrow{\vec{\xi}}^{m n}$ is $\Gamma^{m n}=$ $(m+1)(n+1)$, where $m+n=i$. The maximum would be $i=\Omega+1$ and $m=$ $n=\frac{1}{2}(\Omega+1)$ if $\Omega$ is odd, or $m+1=n=\frac{1}{2}(\Omega+2)$ if $\Omega$ is even. This would lead to Eq. (3.18). For the case of kick-factorizing a 6-dimensional homogeneous Lie transformation of order $i$, each factorization subset should be denoted as $\overline{\vec{\xi}}^{m n o}$, and the required number of bases in the subset is $\Gamma^{m n o}=(m+1)(n+1)(o+1)$, where $m+n+0=i$. Therefore, the minimum required kick number for the 6-dimensional case would be

$$
\Gamma_{\min }=\hat{I}\left(\frac{\Omega+4}{3}\right) \hat{I}\left(\frac{\Omega+5}{3}\right) \hat{I}\left(\frac{\Omega+6}{3}\right),
$$

where $\Omega$ is the order of the map in terms of the Taylor expansion, and $\hat{I}(i / j)$ means integer operation for the division $i / j$. For example, $\Gamma_{\min }=80$ if $\Omega=9$ and $\Gamma_{\min }=125$ if $\Omega=11$.

\subsubsection{Order-by-Order Kick Factorizations}

To kick-factorize the Dragt-Finn factorization map given by Eq. (3.14), we first follow the procedures given in the last section, kick-factorizing the homogeneous Lie transformation $\exp \left(: f_{3}(\vec{z}):\right)$ given as

$$
m_{g, 3}=\prod_{k=1}^{\Gamma} e^{: g_{3, k}\left(x_{k}, y_{k}\right):}
$$

We then Dragt-Finn factorize $m_{g, 3}$ to the 4 th order, given as

$$
m_{g, 3}=\prod_{k=1}^{\Gamma} e^{: g_{3, k}\left(x_{k}, y_{k}\right):}=e^{: f_{3}(\bar{z}):} e^{: h_{4}(\bar{z}):} \ldots
$$


Now let $f_{4}^{\prime}(\bar{z})=f_{4}(\vec{z})-h_{4}(\vec{z})$, and consider the homogeneous Lie transformation $\exp \left(: f_{4}^{\prime}(\bar{z}):\right)$. It can be kick-factorized such that

$$
e^{: f_{i}^{\prime}(\vec{z}): \vec{z}}=\prod_{k=1}^{\Gamma} e^{: g_{i, k}^{\prime}\left(x_{k}, y_{k}\right)}: \vec{z}+\sigma(4) .
$$

Letting $m_{g, 4}=\prod_{k=1}^{\Gamma} e^{: g_{4, k}\left(x_{k}, y_{k}\right)}$, where $g_{4, k}\left(x_{k}, y_{k}\right)=g_{3, k}\left(x_{k}, y_{k}\right)+g_{4, k}^{\prime}\left(x_{k}, y_{k}\right)$, then its Dragt-Finn factorization map up to the 5 th order is given by

$$
m_{g, 4}=e^{: f_{3}(\bar{z}):} e^{: f_{4}(\bar{z}):} e^{: h_{5}(\bar{z}): \ldots .}
$$

Repeating the above for the kick factorization up to the 5th order, then up to the 6 th order, and finally up to the $(\Omega+1)$ th order, we would obtain Eq. (3.15).

\subsubsection{Irwin's Minimization of the Spurious Terms}

The kick factorization procedures discussed in the last sections may result in large spurious terms with orders equal to or larger than $\Omega+1$ in terms of Taylor expansions if the basis set $\overrightarrow{\vec{\xi}}$ and the basis subsets $\overrightarrow{\vec{\xi}}^{m n}$ are not well chosen. In Irwin's original demonstration, he chooses rotational angles $\vec{\theta}_{k}=$ $\left(\theta_{x k}, \theta_{y k}\right)$, for $k=1,2, \ldots \Gamma$ that are equally distributed. Irwin does not choose subsets $\overrightarrow{\vec{\xi}}^{m n}$. For each $m+n=i$ for $i=3,4, \ldots, \Omega+1$, Irwin always uses the same factorization basis set $\overrightarrow{\vec{\xi}}$ consisting of $\Gamma$ bases. Therefore, for most $m, n$ given, there are extra bases. The coefficients of these extra bases are determined by setting constraints that would minimize the high-order spurious terms in the kick factorization map. The reader is encouraged to refer to Irwin's article. ${ }^{17}$

\subsection{Nonlinear Normal Forms}

On many occasions, the nonlinear normal forms of the one-turn maps are desirable. ${ }^{19-27}$ The normal forms can be used for analyzing tune shifts (tune vs. amplitude), resonances, and smear. The differential algebraic normal form of a one-turn map is given by Eq. (3.13), where $m_{f}$ is not given by Eq. (3.14) but is normalized such that ${ }^{27}$

$$
\exp \left(: \sum_{i=2}^{\Omega+1} h_{i}(\vec{J}):\right)=e^{: h(\vec{J}):}=G(\vec{z}) m_{f} G^{-1}(\vec{z})+\sigma(\Omega+1) ;
$$

and so, neglecting high orders $\sigma(\Omega+1), m_{f}$ is given by

$$
m_{f}=G^{-1}(\vec{z}) e^{: h(\vec{J}): G(\vec{z}),}
$$


where the canonical generator, $G(\bar{z})$, is the concatenation of a series of homogeneous Lie transformations given by

$$
G(\vec{z})=\prod_{i=3}^{\Omega+1} e^{: F_{i}(\vec{z}):}
$$

$\vec{J}$ is the vector representing the Courant-Snyder invariants which, taking a transverse map, for example, is given by

$$
\vec{J}^{T}=\left(J_{x}, J_{y}\right) \text { and } J_{x}=\frac{1}{2}\left(x^{2}+p_{x}^{2}\right), \quad J_{y}=\frac{1}{2}\left(y^{2}+p_{y}^{2}\right)
$$

Note that $h_{2}(\vec{J})=-\vec{\mu} \cdot \vec{J}$ and that

$$
e^{: h(\vec{J}):}=\exp (-\vec{\mu} \cdot \vec{J}) \exp \left(: \sum_{i=3}^{\Omega+1} h_{i}(\vec{J}):\right)=\mathcal{R}(\vec{z}) \exp \left(: \sum_{i=3}^{\Omega+1} h_{i}(\vec{J}):\right)
$$

The tune information is contained in the normalized form $\exp (: h(\vec{J}):)$ where $\vec{\mu}$, for example, $\vec{\mu}^{T}=\left(\mu_{x}, \mu_{y}\right)$, are the linear tunes that are modified by the nonlinear effects governed by $\exp \left(: \sum_{i=3}^{\Omega+1} h_{i}(\vec{J}):\right)$. All the information concerning resonances is contained in the canonical generator $G(\vec{z})$.

\subsubsection{Order-by-Order Normalization}

Let

$$
m_{f}=\mathcal{R}(\bar{z}) e^{: f_{3}(\bar{z}):} \mathcal{Q}_{3}(\vec{z}),
$$

where $\mathcal{Q}_{3}(\vec{z}) \vec{z}=\vec{z}+\sigma(3)$. Now consider a canonical transformation given by

$$
\begin{aligned}
m_{3} & =e^{: F_{3}(\vec{z}):} m_{f} e^{-: F_{3}(\vec{z}):} \\
& =e^{F_{3}(\vec{z}):} \mathcal{R}(\vec{z}) e^{: f_{3}(\vec{z}):} \mathcal{Q}_{3}(\vec{z}) e^{-: F_{3}(\vec{z}):} \\
& =\mathcal{R}(\vec{z}) \mathcal{R}^{-1}(\vec{z}) e^{: F_{3}(\vec{z}):} \mathcal{R}(\vec{z}) e^{: f_{3}(\vec{z}):} e^{-: F_{3}(\vec{z}):} e^{: F_{3}(\vec{z}):} \mathcal{Q}_{3}(\vec{z}) e^{-: F_{3}(\vec{z}):} \\
& =\mathcal{R}(\vec{z}) e^{: \mathcal{R}^{-3}(\vec{z}) F_{3}(\vec{z}):} e^{: f_{3}(\vec{z}):} e^{-: F_{3}(\vec{z}):} \mathcal{Q}_{3}^{\prime}(\vec{z}) \\
& =\mathcal{R}(\vec{z}) e^{:\left(\mathcal{R}^{-1}(\vec{z})-\mathcal{I}\right) F_{3}(\vec{z})+f_{3}(\vec{z}):} \mathcal{Q}_{3}^{\prime \prime}(\vec{z}) \\
& =\mathcal{R}(\vec{z}) e^{: h_{3}(\vec{J}):} \mathcal{Q}_{3}^{\prime \prime}(\vec{z})
\end{aligned}
$$

where we have put

$$
\left(\mathcal{R}^{-1}(\vec{z})-\mathcal{I}\right) F_{3}(\vec{z})+f_{3}(\vec{z})=h_{3}(\vec{J})
$$


Thus,

$$
F_{3}(\vec{z})=\frac{1}{I-\mathcal{R}^{-1}(\vec{z})}\left(f_{3}(\vec{z})-h_{3}(\vec{J})\right) .
$$

Note that we use $\mathcal{I}$ instead of 1 to emphasize that $\left(\mathcal{I}-\mathcal{R}^{-1}(\vec{z})\right)$ is a Lie operator, where $\mathcal{I}$ is a unit Lie operator.

Let us defer the discussion of how $h_{3}(\vec{J})$ is chosen and how $F_{3}(\vec{z})$ is obtained. Let us simply assume that $h_{3}(\vec{J})$ and $F_{3}(\vec{z})$ have been given. Then performing the canonical transformation given by Eq. (3.25), we obtain $m_{3}$ and so

$$
\mathcal{Q}_{3}^{\prime \prime}(\vec{z})=e^{-: h_{3}(\vec{J}): \mathcal{R}^{-1}(\vec{z}) m_{3} .}
$$

Note that $\mathcal{Q}_{3}^{\prime \prime}(\bar{z})$ can be Dragt-Finn factorized such that

$$
m_{3}=\mathcal{R}(\vec{z}) e^{: h_{3}(\bar{\jmath}):} e^{: f_{4}(\bar{z}):} \mathcal{Q}_{4}(\vec{z}),
$$

where $\mathcal{Q}_{4}(\vec{z}) \vec{z}=\vec{z}+\sigma(4)$.

Consider the next order canonical transformation given by

$$
\begin{aligned}
& m_{4}=e^{: F_{4}(\bar{z}):} m_{3} e^{-: F_{4}(\bar{z}):} \\
& =e^{: F_{4}(\vec{z}):} \mathcal{R}(\vec{z}) e^{: h_{3}(\vec{J}):} e^{: f_{4}(\vec{z}):} \mathcal{Q}_{4}(\vec{z}) e^{-: F_{4}(\bar{z}):} \\
& =\mathcal{R}(\vec{z}) e^{: \mathcal{R}^{-1}(\vec{z}) F_{4}(\vec{z})} e^{: h_{3}(\vec{J}):} e^{: f_{4}(\bar{z}):} e^{-: F_{4}(\bar{z}):} \mathcal{Q}_{4}^{\prime}(\vec{z}) \\
& =\mathcal{R}(\vec{z}) e^{: h_{3}(\vec{J})+\left(\mathcal{R}^{-1}(\bar{z})-I\right) F_{4}(\bar{z})+f_{4}(\bar{z}):} \mathcal{Q}_{4}^{\prime \prime}(\bar{z}) \\
& =\mathcal{R}(\vec{z}) e^{: h_{3}(\vec{J})+h_{4}(\vec{J}):} \mathcal{Q}_{4}^{\prime \prime}(\vec{z}),
\end{aligned}
$$

where again we have put

$$
\left(\mathcal{R}^{-1}(\vec{z})-\mathcal{I}\right) F_{4}(\vec{z})+f_{4}(\vec{z})=h_{4}(\vec{J})
$$

Thus,

$$
F_{4}(\vec{z})=\frac{1}{\mathcal{I}-\mathcal{R}^{-1}(\vec{z})}\left(f_{4}(\vec{z})-h_{4}(\vec{J})\right)
$$

Repeating the above processes, we finally obtain $f_{i}(\vec{z}), h_{i}(\vec{z})$, and $F_{i}(\vec{z})$ for $i=5,6, \ldots, \Omega+1$, such that

$$
\begin{aligned}
m_{\Omega+1} & =e^{: F_{\Omega+1}(\bar{z}):} \ldots e^{: F_{i}(\bar{z}):} \ldots e^{: F_{3}(\vec{z}):} m_{f} e^{-: F_{3}(\vec{z}):} \ldots e^{-: F_{i}(\vec{z}):} \ldots e^{-: F_{\Omega+1}(\bar{z}):} \\
& =\mathcal{R}(\bar{z}) e^{: h_{3}(\vec{J})+\ldots+h_{i}(\vec{J})+\ldots+h_{\Omega+1}(\vec{J}):} \mathcal{Q}_{\Omega+1}^{\prime \prime}(\vec{z})
\end{aligned}
$$

where

$$
F_{i}(\vec{z})=\frac{1}{\mathcal{I}-\mathcal{R}^{-1}(\vec{z})}\left(f_{i}(\vec{z})-h_{i}(\vec{J})\right)
$$


and

$$
\mathcal{Q}_{\Omega+1}^{\prime \prime}(\vec{z}) \vec{z}=I z+\sigma(\Omega+1)
$$

Neglecting high orders $\sigma(\Omega+1)$, we would obtain the normal form given by Eq. (3.24).

Note that except for $f_{3}(\vec{z})$, which is the same as given by Eq. (3.14), $f_{i}(\vec{z})$ for $i=4,5, \ldots \Omega+1$, are not necessarily the same as given by Eq. (3.14). In the next sections, we shall discuss how $h_{i}(\vec{J})$ and $F_{i}(\vec{z})$, for $i=3,4, \ldots, \Omega+1$, given by Eq. (3.26), are obtained once $f_{i}(\vec{z})$ is obtained.

\subsubsection{Eigenfunctions of the Linear Normal Form}

Since $\left(\mathcal{I}-\mathcal{R}^{-1}(\vec{z})\right)$ in Eq. (3.26) is a Lie operator, it follows that we can obtain $F_{i}(\vec{z})$ if we can decompose $f_{i}(\vec{z})$ and $h_{i}(\vec{J})$ into polynomials of the eigenfunctions of $\mathcal{R}^{-1}(\vec{z})$ or $\mathcal{R}(\vec{z})$. For simplicity without losing generality, we shall consider a transverse map. Then

$$
\mathcal{R}^{-1}(\vec{z})=e^{: \vec{\mu} \cdot \vec{J}:}=e^{: \mu_{x} J_{x}+\mu_{y} J_{y}:},
$$

where $J_{x}=\frac{1}{2}\left(x^{2}+p_{x}^{2}\right)$ and $J_{y}=\frac{1}{2}\left(y^{2}+p_{y}^{2}\right)$. It is clear that $\hat{x}_{ \pm}=\frac{1}{\sqrt{2}}\left(x \pm i p_{x}\right)$ and $\hat{y}_{ \pm}=\frac{1}{\sqrt{2}}\left(y \pm i p_{y}\right)$ are eigenfunctions of the Lie operators : $J_{x}:$ and $: J_{y}:$, respectively, as we have

$$
\begin{aligned}
& : J_{x}: \hat{x}_{ \pm}=\left[\frac{1}{2}\left(x^{2}+p_{x}^{2}\right), \frac{1}{\sqrt{2}}\left(x \pm i p_{x}\right)\right]= \pm i \hat{x}_{ \pm}, \\
& : J_{y}: \hat{y}_{ \pm}=\left[\frac{1}{2}\left(y^{2}+p_{y}^{2}\right), \frac{1}{\sqrt{2}}\left(y \pm i p_{y}\right)\right]= \pm i \hat{y}_{ \pm} .
\end{aligned}
$$

Thus, any monomial given by $\hat{x}_{+}^{l} \hat{x}_{-}^{m} \hat{y}_{+}^{n} \hat{y}_{-}^{o}$, where $l, m, n, o$ are integers, is an eigenfunction of $\mathcal{R}^{-1}(\vec{z})$. In fact,

$$
\begin{aligned}
\left(\mathcal{I}-\mathcal{R}^{-1}(\vec{z})\right) \hat{x}_{+}^{l} \hat{x}_{-}^{m} \hat{y}_{+}^{n} \hat{y}_{-}^{o} & =\left(\mathcal{I}-e^{: \vec{\mu} \cdot \vec{J}:}\right) \hat{x}_{+}^{l} \hat{x}_{-}^{m} \hat{y}_{+}^{n} \hat{y}_{-}^{o} \\
& =\left(1-e^{i\left\{(l-m) \mu_{x}+(n-o) \mu_{y}\right\}}\right) \hat{x}_{+}^{l} \hat{x}_{-}^{m} \hat{y}_{+}^{n} \hat{y}_{-}^{o} .
\end{aligned}
$$

\subsubsection{Decomposition of the Homogeneous Polynomial}

It can be proved that the real homogeneous polynomial $f_{i}(\vec{z})$ can be decomposed as follows:

$$
f_{i}(\vec{z})=f_{i}\left(x, p_{x}, y, p_{y}\right)=\sum_{l+m+n+o=i} c_{l m n o} \hat{x}_{+}^{l} \hat{x}_{-}^{m} \hat{y}_{+}^{n} \hat{y}_{-}^{o},
$$


where $c_{l m n o}$ is a complex number in general. Therefore, it seems that we would have

$$
\frac{1}{\mathcal{I}-\mathcal{R}^{-1}(\bar{z})} f_{i}(z)=\sum_{l+m+n+o=i} \frac{c_{l m n o}}{1-e^{i\left\{(l-m) \mu_{x}+(n-o) \mu_{y}\right\}}} \hat{x}_{+}^{l} \hat{x}_{-}^{m} \hat{y}_{+}^{n} \hat{y}_{-}^{o} .
$$

However, the denominators $1-e^{i\left\{(l-m) \mu_{x}+(n-o) \mu_{y}\right\}}$ will be 0 if $(l-m) \mu_{x}+(n-$ o) $\mu_{y}=0$. Therefore, to obtain $F_{i}(\vec{z})$ given by Eq. (3.26), we would take terms with $l=m$ and $n=o$ away from $f_{i}(\vec{z})$. Choosing

$$
h_{i}(\vec{J})=\sum_{m+n=i / 2} c_{m m n n}\left(\hat{x}_{+} \hat{x}_{-}\right)^{m}\left(\hat{y}_{+} \hat{y}_{-}\right)^{n}=\sum_{m+n=i / 2} c_{m m n n} J_{x}^{m} J_{y}^{n}
$$

Eq. (3.26) would be solvable if the tunes $\mu_{x}=2 \pi \nu_{x}$ and $\mu_{y}=2 \pi \nu_{y}$ are well chosen, as they should be for a good storage ring. Note that $h_{i}(\vec{J})=0$ for all $i=$ odd number. Please also note that $F_{i}(\vec{z})$ given by Eq. (3.26) can be evaluated without employing complex differential algebras. ${ }^{27}$

\subsubsection{Nonlinear Resonances}

Note that occasionally $F_{i}(\bar{z})$ given by Eq. (3.26) will have terms with

$$
(l-m) \mu_{x}+(n-o) \mu_{y}=2 \pi(l-m) \nu_{x}+2 \pi(n-o) \nu_{y} \approx 2 \pi p,
$$

where $l, m, n, o, p$ are all integers with $l \neq m$ and $n \neq o$. These cases correspond to nonlinear resonances.

\section{Long-Term Trackings}

As discussed in Chapter 2, storage rings are complicated nonlinear machines that make detailed analytical studies almost impossible. Computer simulations of tracking particle trajectories are thus widely employed. There are various tracking programs with various algorithms in the accelerator physics community. Most of these programs are surveyed by Keil. ${ }^{28}$

In this chapter, we shall discuss applications of differential algebraic maps for long-term stability studies of the storage rings. For comparison purposes and as a complement to Chapter 2, we shall begin with a discussion of long-term trackings of the SSC using a systematic program named Teapot ${ }^{29}$ and its later version, the vectorized element-by-element tracking program Ztrack. ${ }^{30}$ We shall then proceed with the discussion of how one-turn differential-algebraic maps are constructed and how they are applied for long-term trackings. In particular, we shall discuss the SSC lattice with the use of a post-Teapot, differential-algebraic map extraction program, Zmap. ${ }^{31}$ 


\subsection{Element-by-Element Trackings}

Conventionally, particle trajectories are advanced at least one step in each of the magnet elements; thus many steps are required in one turn of the storage ring. For example, in tracking with the program Teapot, each particle receives one or more multipole kicks as it passes a magnet element. Between muitipole kicks are drifts. Such a tracking procedure is called element-by-element tracking. These element-by-element trackings are the foundations that establish numerical studies of the storage rings and the accelerators.

Recently intensive element-by-element long-term trackings have been performed for the SSC aperture study. ${ }^{32-34}$ Such extensive and elaborate efforts have been required because of the unique scale of the SSC, particularly its budget and its size. First, the budget scale of the SSC makes it imperative to look hard for superconducting magnets with the smallest possible transverse size that can offer adequate safety margin for operation. ${ }^{*}$ Therefore, the SSC is designed to be more nonlinear than the previous superconducting synchrotron, the Tevatron at Fermi National Accelerator Laboratory. This high nonlinearity makes long-term stability of the SSC unpredictable through analytical approaches or standard, short-term tracking studies, such as the study of smear. Secondly, the large size ( 87 kilometers circumference) of the SSC and the relatively slow final booster ring require a long injection time to fill the collider rings with proton bunches. The total time for the SSC injection is about 50 minutes, which is equivalent to about 10 million turns around the collider ring. There is no acceleration at injection, although there are synchrotron oscillations in the proton bunches. This is a critical stage in the operation of the SSC because the transverse emittance of the proton beam is at its maximum. Once the proton beam is accelerated after completion of injection, its transverse emittance will be adiabatically damped.

\subsubsection{Numerical Description}

In these tracking studies, the systematic program Teapot was used for preparing the final corrected nonlinear lattice file named "Zfile," which was subsequently input into Ztrack for vectorized particle trackings. Numerical steps are briefly described as follows.

* A superconducting magnet with a larger transverse size will provide a larger cross section of uniform magnetic field for charged-particle motion around the ring, but it will cost more. A magnet with a smaller transverse size, although less expensive, will have larger multipole errors and thus be more nonlinear. 
Step 1. Preparation of the Linear Lattice. The SSC linear lattice $\mathbf{e}^{35}$ is designed using a program called Synch. ${ }^{36} \mathrm{~A}$ standard "MAD" ${ }^{37}$ input that describes a thin-lens representation of the SSC injection linear lattice is then prepared. Teapot then reads in the input and converts all thick elements to thin ones. Note that all the correctors are included in the lattice.

Step 2. Addition and Correction of Random and Systematic Multipole Errors. Once the linear lattice is defined, random and systematic multipole errors are added to the dipoles in the lattice. Table 4.1 presents as an example two sets of random and systematic errors representing the 4-cm and the $5-\mathrm{cm}$-coildiameter superconducting magnet dipoles. In this study, there are no corrections other than the use of chromaticity sextupoles and tune quadrupoles to correct

Table 4.1. Random, Systematic, and Orbit Errors and Their Corrections

\begin{tabular}{lll}
\hline Case & $4 \mathrm{~cm}$ & $5 \mathrm{~cm}$ \\
Half Cell Length & $90 \mathrm{~m}$ & $90 \mathrm{~m}$ \\
Injection Energy & $2 \mathrm{TeV}$ & $2 \mathrm{TeV}$ \\
Dipole Coil Diameter & $4 \mathrm{~cm}$ & $5 \mathrm{~cm}$ \\
Random Errors & $\sigma_{b_{2}}=0.4$ & $\sigma_{b_{2}}=0.272$ \\
& $\sigma_{b_{3}}=0.3$ & $\sigma_{b_{3}}=0.18$ \\
& $\sigma_{b_{4}}=0.7$ & $\sigma_{b_{3}}=0.35$ \\
& $\sigma_{a_{2}}=0.6$ & $\sigma_{a_{2}}=0.41$ \\
& $\sigma_{a_{3}}=0.7$ & $\sigma_{a_{3}}=0.41$ \\
& $\sigma_{a_{4}}=0.2$ & $\sigma_{a_{4}}=0.1$ \\
Systematic Errors & $b_{2}=-3.0$ & $b_{2}=-1.9$ \\
& $b_{3}=0$ & $b_{3}=0$ \\
& $b_{4}=0.2$ & $b_{4}=0.09$ \\
Misalignment Errors & $b_{6}=-0.05$ & $b_{6}=-0.017$ \\
& $\sigma_{a_{0}}=0.000834$ & $\sigma_{a_{0}}=0.000834$ \\
& $\sigma_{b_{0}}=0.0012$ & $\sigma_{b_{0}}=0.0012$ \\
& $\sigma_{\theta}=0.0005$ rad (dipole) & $\sigma_{0}=0.0005$ rad (dipole) \\
Corrections & $\sigma_{x}=\sigma_{y}=0.001 \mathrm{~m}$ & ${ }^{*} \sigma_{x}=\sigma_{y}=0.001 \mathrm{~m}$ \\
& Use only chromaticity & Use only chromaticity \\
& sextupoles to correct & sextupoles to correct \\
Tunes & the systematic $b_{2} ;$ & the systematic $b_{2} ;$ \\
RMS orbit distortion & $(0.285,0.265)$ & $\sigma_{b_{2}}$ reduced to 0.272 \\
& $\sigma_{b_{2}}$ reduced to 0.4 & $(0.285,0.265)$ \\
& 1 mm & 1 mm \\
\hline
\end{tabular}

*For dipoles, quadrupoles, and beam monitors. 
the systematic $b_{2}$ and the working tunes. (Note also that the normal and skew $b_{1}$ and $a_{1}$ are set to zero with the assumption of decoupling.)

Step 3. Addition of Misalignments and Correction of Orbit. After the random and systematic multipole errors are added to the lattice and the chromaticities and the tunes are adjusted to the desired values, the next step is the addition of random errors due to misalignments (see Table 4.1). These errors include: (1) random dipole errors $\left(\sigma_{a_{0}}\right.$ and $\left.\sigma_{b_{0}}\right)$; (2) rotations $\left(\sigma_{\theta}\right)$; and (3) displacements $\left(\sigma_{x}\right.$ and $\left.\sigma_{y}\right)$. After the addition of errors due to misalignments, the orbit is corrected to an rms orbit deviation of $1 \mathrm{~mm}$ in both the horizontal and vertical planes with respect to the reference orbit. In some other cases not shown here, decoupling is simultaneously performed between the horizontal and vertical planes. The above numerical steps are all performed on a SUN workstation.

Step 4. Retuning. Once the orbit is corrected to a desired value of rms orbit deviation, the chromaticities and the working tunes are readjusted to desired values. A final lattice file, "Zfile," is then written and transferred to a Cray computer at the DOE National Energy Research Supercomputer Center (NERSC).

Step 5. Tracking. Ztrack was used to track particles in supercomputers. Ztrack reads in the final lattice file "Zfile" and, based on the command input file "Zcmd," Ztrack adds an $\mathrm{rf}$ cavity if synchrotron oscillations are required. A TOPDRAWER ${ }^{38}$ file is written for the survival plot at the end of the tracking. A restart file is written every "ntsave" turns and at the end of tracking in order to protect from loss of tracking information due to unexpected computer problems, to retrieve information during the tracking, and to continue turn tracking after the requested turns of tracking have been performed.

\subsubsection{Survival Plots}

Interested readers are referred to the site-specific conceptual design report ${ }^{2}$ of the SSC for more long-term tracking results and to an article by $\mathrm{Chao}^{39}$ for more long-term tracking analysis. What follows here is a typical result obtained from Ztrack long-term tracking for the SSC aperture study. (Magnet and orbit errors are shown in Table 4.1.)

Figure 4.1 shows survival plots for 100,000-turn tracking from Ztrack. Two 2- $\mathrm{TeV}$ injection lattices, one with 4-cm-coil-diameter dipoles and the other with 5-cm-coil-diameter dipoles, were studied. The two lattices have the same random seed for the assignment of random errors. The only difference between the two lattices is in the multipole content, which is due to the difference in magnet aperture. The $\mathrm{rf}$ cavity system was turned on to maintain bunching. All particles were initiated with an identical synchrotron oscillation amplitude of $3 \sigma_{\mathrm{rms}}\left(\sigma_{\mathrm{rms}}\right.$ : rms half bunch length) but with an appropriate distribution of initial horizontal 


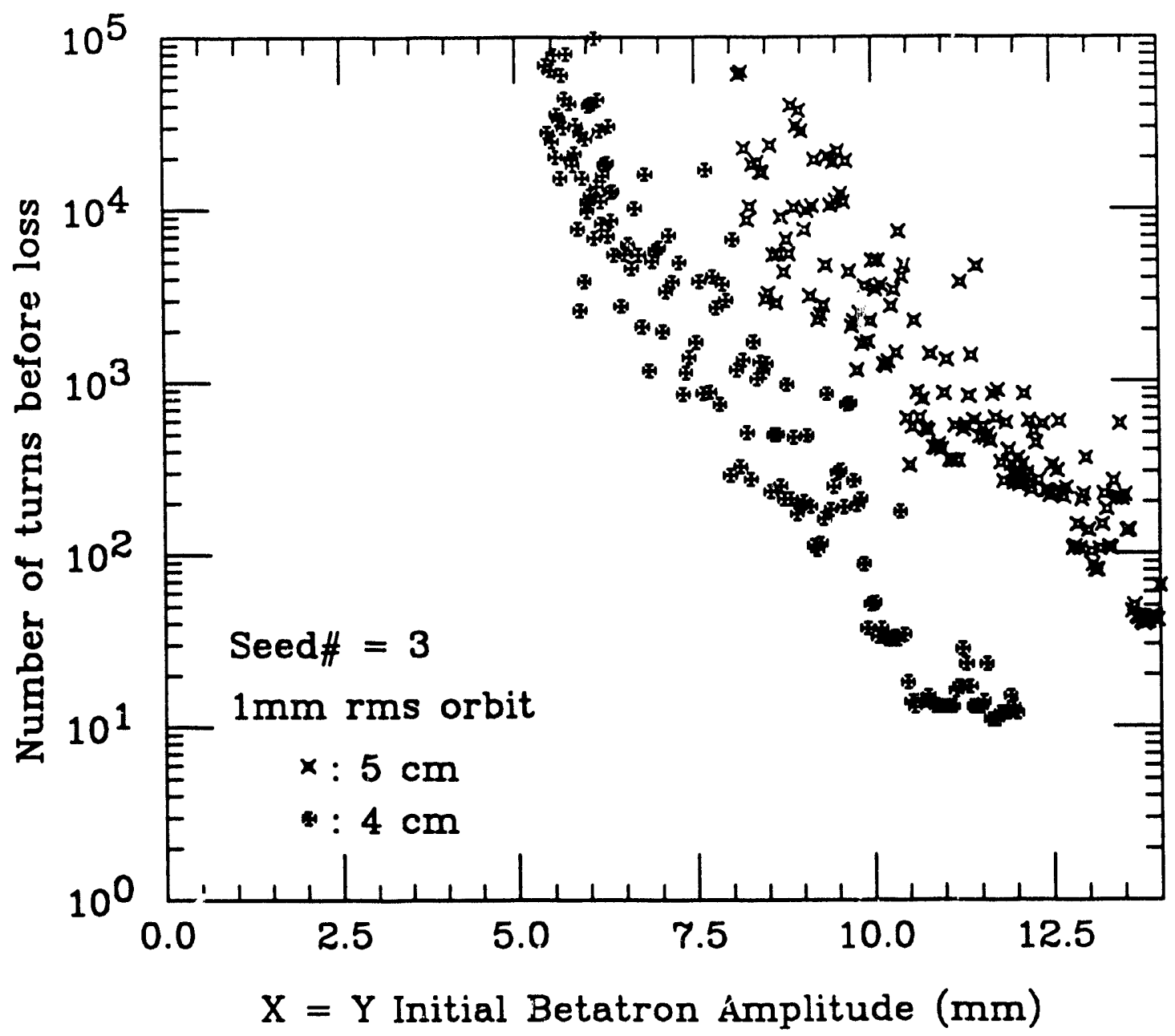

Figure 4.1. 100,000-turn survival plots for two 2-TeV SSC injection lattices, comparing the dynamic aperture for two different magnet apertures-one with 4-cm-coil-diameter dipoles and the other with 5-cm-coil-diameter dipoles. With the 4-cm-coil-diameter dipoles, no particles with initial $x$ displacement amplitude of $<5.3 \mathrm{~mm}$ were lost. With the 5-cm-coil-diameter dipoles, no particles with initial $x$ displacement amplitude of $<8.1 \mathrm{~mm}$ were lost. By increasing the magnet aperture, the dynamic aperture for 100,000 turns enlarges from about $5.3 \mathrm{~mm}$ to about $8.1 \mathrm{~mm}$ in radius, and the machine's linearity improves. This plot shows only the protons that were lost before 100,000 turns are reached.

$\left(x\right.$-axis) and vertical ( $y$-axis) displacements $\left(y / \sqrt{\beta_{y}}=x / \sqrt{\beta_{x}}\right.$ and $p_{x}=p_{y}=0$ at injection, which is at the middle of a horizontal focusing quadrupole).

While many of the particles with higher displacement amplitudes were lost in the earlier turns, all of the particles with initial $x$-displacement amplitudes lower than $5.3 \mathrm{~mm}$ for the 4-cm-coil-diameter dipole case and lower than $8.1 \mathrm{~mm}$ for the 5-cm-coil-diameter dipole case survived. The dynamic apertures for 
100,000 turns are thus about $5.3 \mathrm{~mm}$ and $8.1 \mathrm{~mm}$ in radius for the $4-\mathrm{cm}$-coildiameter dipole case and the 5-cm-coil-diameter dipole case, respectively.

It should be noted that even though Ztrack is optimized, because of the large size of the SSC collider rings, obtaining such a survival plot ( $10^{5}$ turns) for each of the SSC collider lattices requires about 20 hours Cray XMP CPU time or slightly less CPU time if Cray 2 or Cray YMP instead of Cray XMP is used. Therefore, it is virtually impossible to obtain a 10-million-turn (the lifetime of an SSC injection lattice) survival plot for the SSC with such an element-by-element tracking approach.

\subsection{One-Turn-Map Trackings}

As discussed in the previous section, a conventional element-by-element tracking method is virtually impossible for the desired lifetime (10 million turns) in the SSC injection lattice. Mapping techniques are currently among alternatives under consideration for achieving such a task. In this section, we shall first discuss how one-turn maps are constructed. We shall then compare a sample of map tracking results of the SSC injection lattice with the element-by-element tracking results.

\subsubsection{Construction of One-Turn Maps}

One of the most practical one-turn maps that can be constructed directly and efficiently is the one-turn Taylor map. Without losing generality, we shall, in this section, take examples and use some Fortran language terminology to illustrate the one-turn Taylor map construction. First, we shall consider a simple case: a straight beam line that consists of periodic sections. Each section consists of "nelem" perfectly aligned, thin-magnet elements, each of which is followed by a drift. Each of the thin elements is assumed to contain normal quadrupole, normal sextupole, and normal octupole kicks only (no skews and no errors); that is, there is no coupling between the two transverse degrees of freedom. For simplicity, we shall consider only extraction of the horizontal, one-pciicd, on-momentum Taylor map and assume that we are interested in the 9th-order map. The core part of the regular element-by-element tracking codes for the on-momentum particles in one periodic section is as follows. (Illegal Fortran variable names may be used throughout this section for clarity.)

$$
\begin{gathered}
\text { - Initialization } \\
x=x_{i} \\
p_{x}=p_{x, i}
\end{gathered}
$$




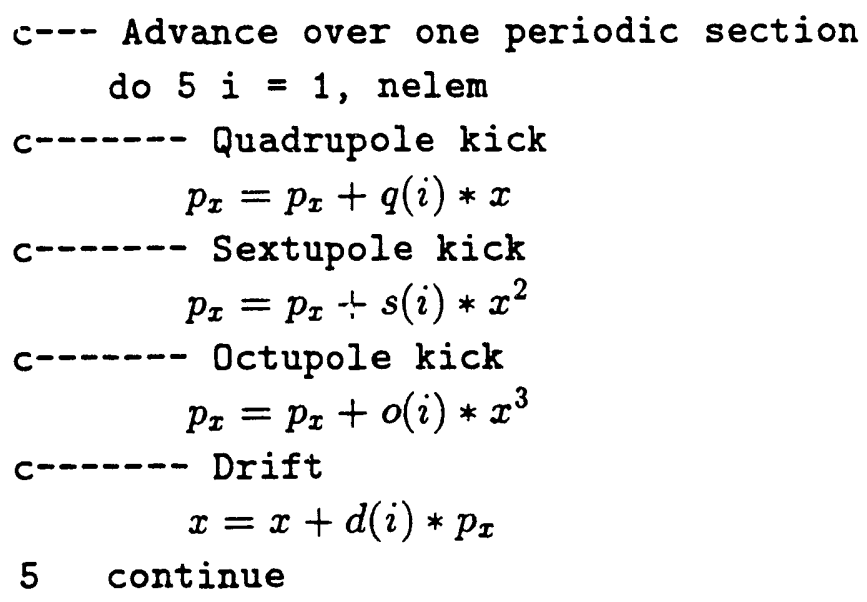

Note that $q(i), s(i), o(i)$ and $d(i)$ for $i=1,2, \ldots$, nelem, are assumed to have been calculated and stored. Here's how to convert these element-by-element tracking codes into the one-period 9 th-order Taylor map extraction codes. First, the declaration statement

$$
\text { "parameter ( } n v=2, n o=9, n m=55) "
$$

is added in the codes where appropriate, where " $n v$ " is the phase-space dimensions, "no" is the desired order of the Taylor map, and "nm" is the number of monomials that can be calculated with use of the formula " $\mathrm{nm}=$ $(2+9) ! /(2 ! 9 !) "$. Second, a two-dimensional array "uu(nm,2)" is declared for representing the coefficients $\vec{u}(\vec{k})$ of the 2-D, 9th-order VPS (the Taylor map):

$$
\vec{U}(\vec{z})=\sum_{k=0}^{9} \vec{u}(\vec{k}) \vec{z}^{\vec{k}} .
$$

For convenience, a mnemonic common block is also declared for the two components of the VPS uu:

$$
\begin{aligned}
& \text { "dimension uu(nm, } 2) ", \\
& \text { "common } u_{x}(\mathrm{~nm}), u_{p}(\mathrm{~nm}) ", \\
& \text { "equivalence }\left(u_{x}, \mathrm{uu}\right) " ;
\end{aligned}
$$

that is, $u_{x}(*), u_{p}(*)$ share the same memories with uu $(*, 1)$, uu $(*, 2)$, respectively. Note that $u_{x}(*), u_{p}(*)$ are actually declared for the rays $\left(x^{\prime}, p_{x}^{\prime}\right)$ at a given position along the beam line as power series expansions of the rays $\left(x, p_{x}\right)$ at the initial position. One auxiliary PS array is also declared as follows:

$$
\text { "dimension } u_{t}(\mathrm{~nm}) " \text {. }
$$

Once declarations of arrays for the PSs are appropriately made, an executable statement

$$
\text { "call zpprep (nv, no, nmuse, np)" }
$$


is added to the program before any subroutine of the Zlib is called. Again, " $n v$ " is the number of variables for the PS, which is 2 in this case; "no" is the desired order; "nmuse" is the returned value for the total number of monomials, which should be equal to "nm"; and "np" is the number of particles to be tracked through the map, which should be set to 0 to avoid unnecessary dynamic memories in this case. This statement tells Zlib to allocate necessary minimum dynamic memory for setting up all the required data-structure integer pointers and for the internally required minimum auxiliary PSs and VPSs. After this statement, all the subroutines in the sub-library ZPLIB of the Zlib can be invoked. Now we are ready to convert the core part of the codes for single-particle trackings given above into map extraction codes. The starting procedure is to assign a Taylor map (VPS) that maps the rays at the initial position to the rays at the initial position itself. Such a map is an identity map; that is, the initial Taylor map is a unit nv-dimensional VPS given by

$$
\vec{U}(\vec{z})=\vec{I}(\vec{z})=\vec{z}
$$

Therefore, the two initialization statements for element-by-element tracking codes given above are replaced with the unit VPS assignment statement

$$
\text { "call zpunit (uu,no)". }
$$

Recalling that " $u_{x}(*)$ ", " $u_{p}(*)$ " share memories with "uu $(*, 1)$ ", "uu $(*, 2)$ " respectively, the variables " $x$ " and " $p_{x}$ " inside the "do $5 i=1$, nelem" loop are ready to be replaced by the PSs " $u_{x}$ " and " $u_{p}$ ". The corresponding differential algebraic subroutines in Zlib are then called to achieve the operations. The core part for the map extraction codes follows.

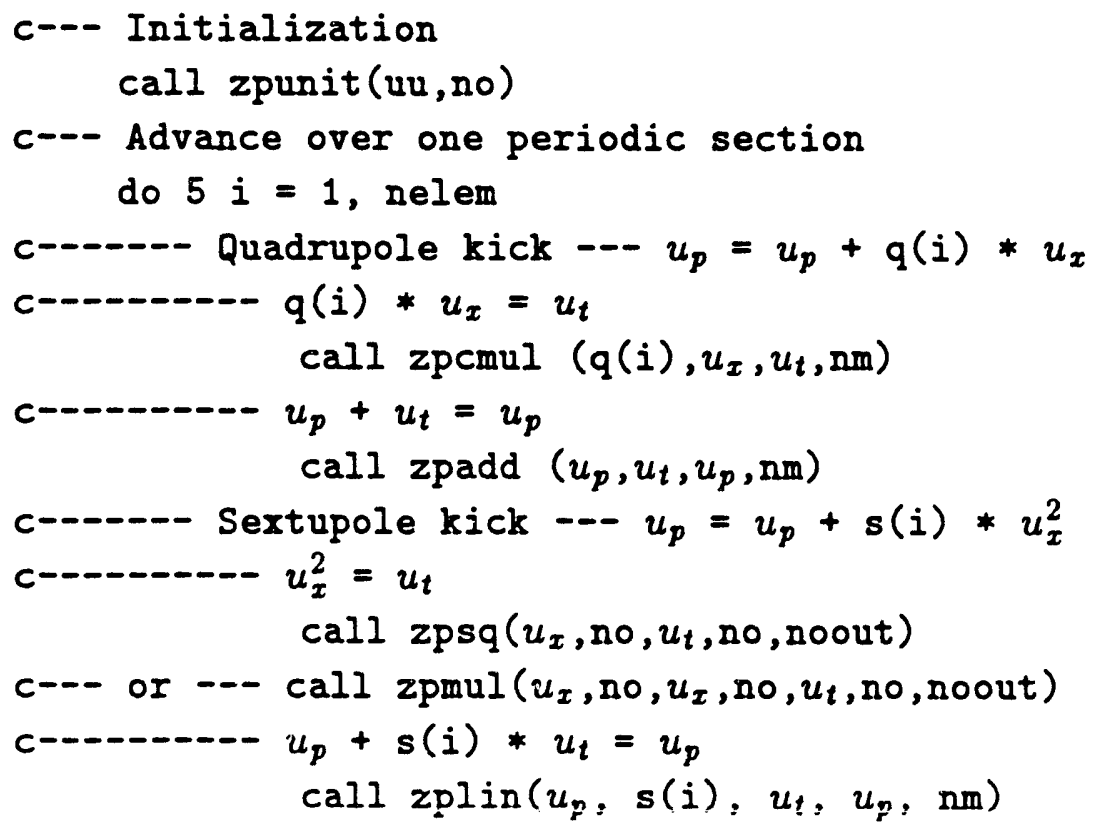




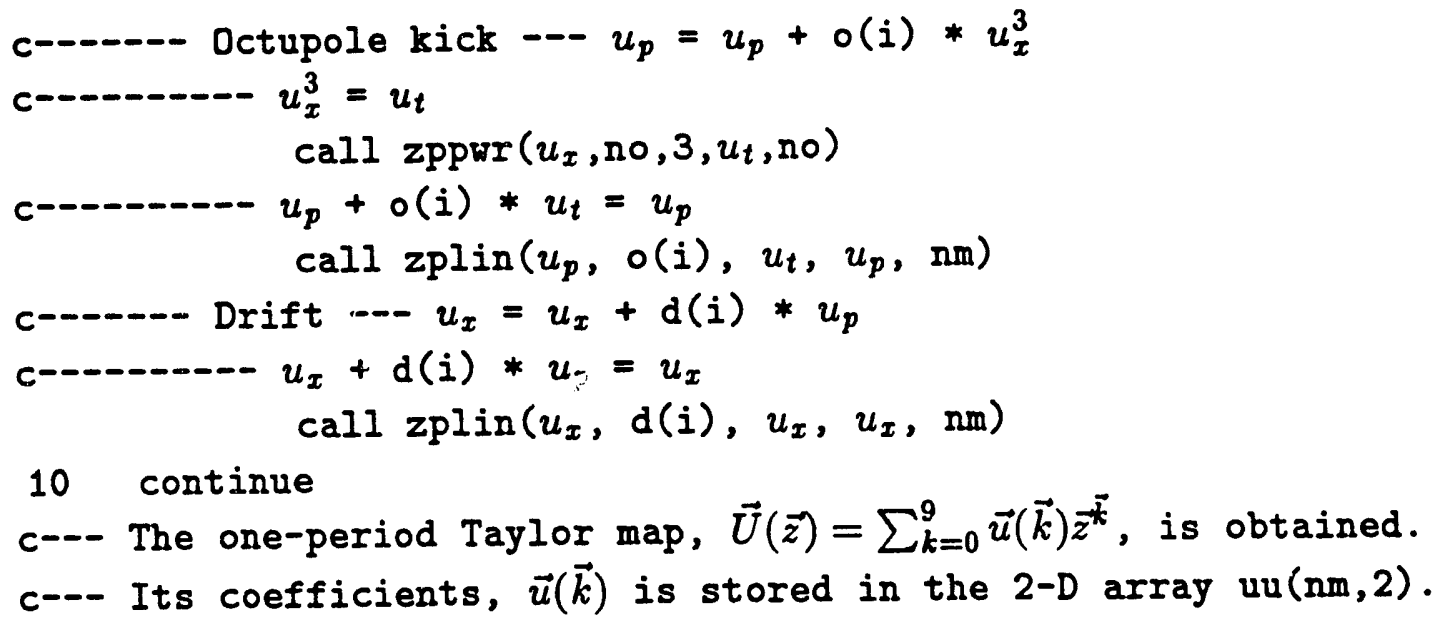

The above calling statements for differential algebraic operations should be self-explanatory. Interested readers may further refer to the Zlib manual. ${ }^{11}$

In some cases, such as this one, the number of variables and the order of all the PSs and VPSs involved in the program are identical and fixed. Under such a circumstance, all the parameters in the PS and VPS operational calling statements related to the number of variables, the orders, and the number of monomials such as "no", "noout", and "nm" shown above are avoidable. A simple precompiler can be used to translate the PS and VPS operations into the corresponding calling statements of the PS and VPS operational subroutines.

There are several practical examples of converting existing element-byelement tracking programs into one-turn Taylor map extraction programs for storage rings. ${ }^{31,40-42}$ Among these is the program $\mathrm{Zmap} .{ }^{31} \mathrm{Zmap}$ was developed for extracting one-turn Taylor maps from the systematic, circular accelerator program Teapot; ${ }^{29}$ it has been used to extract various one-turn Taylö maps for the SSC lattices. It is converted from the post-Teapot tracking program Ztrack to take advantage of the available setup of the two necessary input files. One is the machine file named Zfile provided by Teapot, which describes the nonlinear lattice. The other is the command file named $\mathrm{Zcmd}$, which in $\mathrm{Zmap}$ describes how the one-turn map is to be extracted, e.g., the order and the dimension of the Taylor map. The conversion process is somewhat similar to the above simple case, although it is lengthier. Arithmetic operations for initializations, kicks, and drifts of Teapot are directly translated into PS or VPS operations of Zlib through a calling statement of the corresponding multi-variable polynomial operation subroutines of Zlib. Most often, Zmap extracts orre-turn Taylor maps with respect to the nominal closed orbit (usually distorted from the desired orbit). If requested by the command file $\mathrm{Zcmd}, \mathrm{Zmap}$ will also fird the dispersed (energy-associated) closed orbit and then extract a one-iurn map with respect to the dispersed closed orbit. Note that the dispersed closed orbit is expressed as a polynomial of the energy deviation, $\delta$, to a specified order. 
Zmap was developed with the hope that each of the extracted maps would satisfy two fundamental properties: (a) it must be simpler than the original element-by-element description of the nonlinear lattice so that enormous computer time can be saved for the study of the storage rings, and (b) all (or certain) important information in the original element-by-eiement nonlinear static lattice must be included; that is, the one-turn map must represent the original elementby-element lattice well enough for specific studies (though it cannot represent the original lattice exactly).

Once a one-turn, high-order Taylor map is obtained, it can be directly used for particle trackings, but with care. It can also be symplectified by conversion into a series of homogeneous Lie transformations (a Dragt-Finn factorization map). These Lie transformations can be transferred back to even higher-order Taylor maps for long-term trackings. They can also be converted into normal forms for various analyses and into kick factorizations for symplectic trackings. The series of homogeneous Lie transformations also can be combined into a single, non-homogeneous Lie transformation through use of the $\mathrm{CBH}$ theorem. The one-turn, single, non-homogeneous Lie transformation may also be obtained directly for special cases by first transferring each kick or each related operation of the tracking codes into a Lie transformation and then combining, through the $\mathrm{CBH}$ theorem, all the Lie transformations into one. ${ }^{43,44}$ However, such a method has not yet proved to be efficient and practical for most of the general applications involved with orbit distortion.

In the following sections, we shall discuss the Taylor maps in more detail. Other forms of maps are further discussed in Chapter 6.

\subsubsection{The Taylor Maps}

If there is no truncation involved with the extraction of a one-turn Taylor map, then the one-turn Taylor map is not only accurate in carrying the lattice information but is also symplectic if it is extracted from a symplectic tracking program. However, because of limited computer memory and speed, truncation of high orders of a one-turn Taylor map is almost necessary for every practical case. For example, without truncation a one-turn Taylor map extracted with Zmap for the SSC injection lattice would be at least an order of $6^{10000}$, where 6 is assumed to be the maximum order of the multipole errors carried in the numerical calculation for each of the magnet elements, and 10000 is assumed to be the minimum number of elements. Therefore, a Taylor map is not only less than perfectly accurate but is also not symplectic in general because of the truncation of the high orders in the map. Would it then be simple to make a tentative conclusion that the one-turn Taylor map is not suitable for long-term trackings? The truth is that there is no perfect accuracy or perfect symplecticity where computer calculations are involved; even a symplectic tracking program 
is not perfectly symplectic because of round-off errors. Therefore, one would rather talk about the "degree" of accuracy and the "degree" of symplecticity. For example, a $10^{7}$-turn tracking of a particle with a Taylor map of the SSC injection lattice would require a higher degree of accuracy and a higher degree of symplecticity and thus a higher truncation order of the Taylor map than its corresponding $10^{6}$-turn tracking.

Having established the concept of the "degree" of accuracy for carrying the lattice information and "degree" of symplecticity for preserving the conservativeness of the system for a truncated differential algebraic one-turn Taylor map, two questions naturally emerge:

Question (Q-1): To what order should the differential algebraic one-turn Taylor map be extracted for a given nonlinear lattice such that it is both accurate enough and symplectic enough for a specified study, such as the study of dynamic aperture to a given turn?

Question (Q-2): Does a given differential algebraic one-turn Taylor map truncated at a certain order have a higher degree of accuracy or a higher degree of symplecticity, and what are the implications?

These questions will be answered as we proceed to discuss the long-term trackings performed with the differential-algebraic Taylor maps for the SSC injection lattices. ${ }^{45-47}$

\subsubsection{Taylor Map Trackings}

For many years one question has remained unanswered: can one-turn Taylor maps be obtained that are suitable for long-term stability studies of storage rings and, in particular, of the SSC? Since the nonlinear effects in the SSC (or storage rings in general) are due to chromaticities, magnet errors, and orbit errors and their associated corrections-which must be small enough compared to the leading linear effects in the region of interest-there must exist a oneturn Taylor map, truncated at a suitably high order, that is accurate enough to represent the lattice. The question, therefore, is not whether the one-turn Taylor map works, but how high the truncated order of a Taylor map should be in order to ensure its reliability in terms of accuracy and symplecticity. One simple way of checking this is to use the Taylor map for particle tracking. The tracking formula is given by

$$
\vec{z}_{i}=m \vec{z}_{i-1}=\vec{U}\left(\vec{z}_{i-1}\right)=\sum_{k=1}^{\Omega} \vec{u}(\vec{k}) \overrightarrow{z_{i-1}^{*}},
$$

where the coefficients $\vec{u}(\vec{k})$ are kept constant, and the turn-by-turn phase-space coordinates $\vec{z}_{i}$ are obtained by substituting the previous turn phase-space coordinates $\vec{z}_{i-1}$ into the truncated multi-variable polynomial of order $\Omega$. Taylor map tracking subroutines in Zlib can be called to perform these map trackings. 
Shown in Figure 4.2 are two phase-space $\left(x, p_{x}\right)$ plots for 400 turns, in the same frame-one from Ztrack element-by-element tracking for the $5-\mathrm{cm}$ diameter dipole SSC injection lattice discussed in Section 4.1.2, and the other from its associated 12th-order Taylor map tracking. A particle is launched with a betatron amplitude of $8.1 \mathrm{~mm}$ in each of the two trackings. Note that the dynamic aperture of this injection lattice is about $8.1 \mathrm{~mm}$. The two phase-space plots are hardly distinguishable within the resolution of this figure. Indeed, the accuracy of one-turn Taylor map tracking for this case is about 8 digits compared to its associated element-by-element tracking.

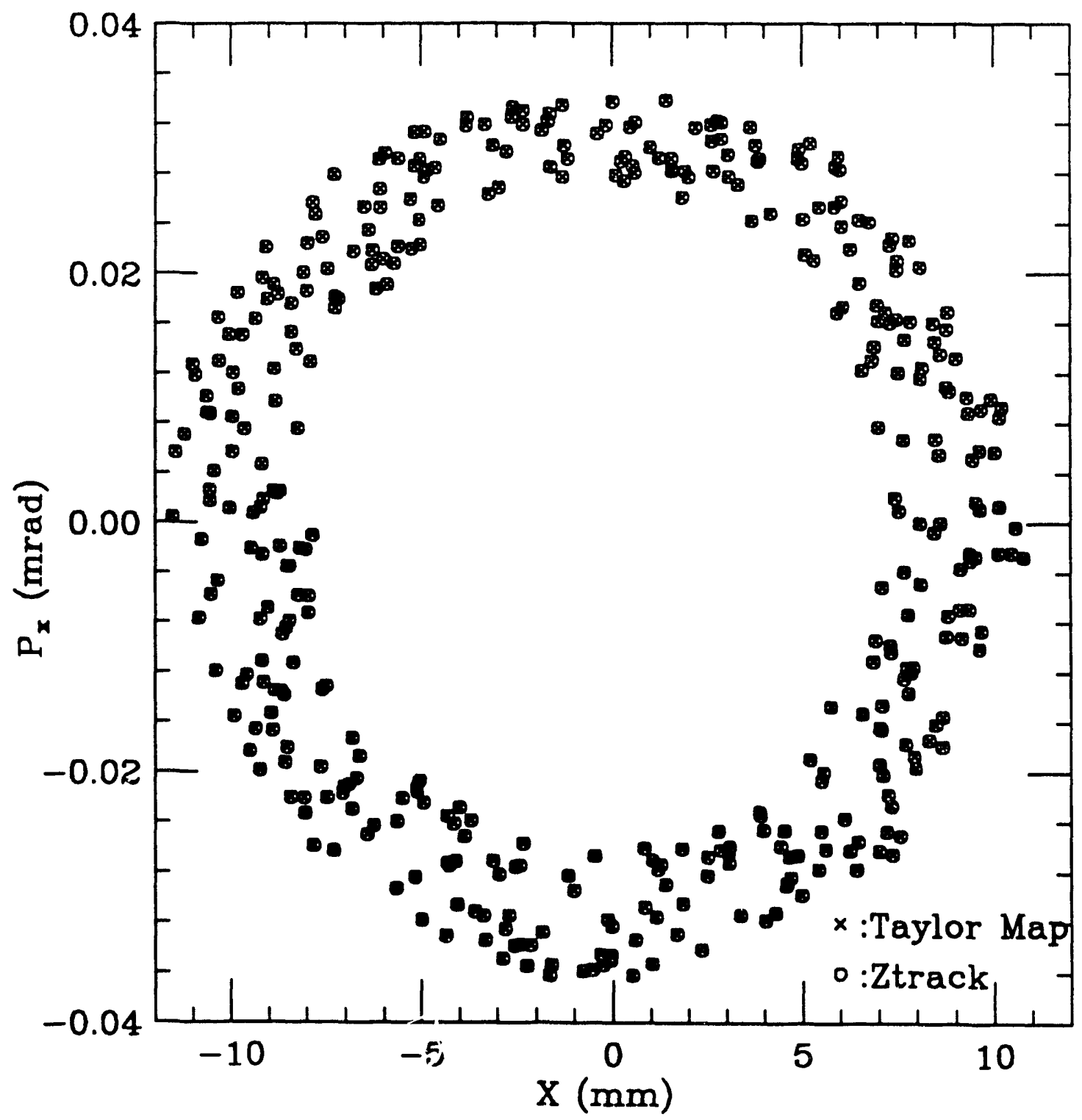

Figure 4.2. Turn-by-turn phase-space plots of an element-by-element tracking of the 5-cm-diameter dipole SSC injection lattice and its associated 12th-order Taylor map tracking for 400 turns. 
Figure 4.3 shows two survival plots, in the same frame, comparing tracking data for 100,000 turns from "Ztrack" element-by-element tracking for the same 5-cm-diameter dipole SSC injection lattice and from its associated 12thorder one-turn Taylor map tracking. Although the map tracking results and the element-by-element tracking results are not identical, they agree in the global behavior. Both show the same dynamic aperture, $8.1 \mathrm{~mm}$ at $10^{5}$ turn.

It is costly to extend to a million-turn survival plot for the SSC injection lattice with the element-by-element tracking program (about 180 hours of Cray 2 CPU time with Ztrack), but not with the one-turn Taylor map tracking (about 5 hours of Cray 2 CPU time for a 14th-order Taylor map tracking with a routine

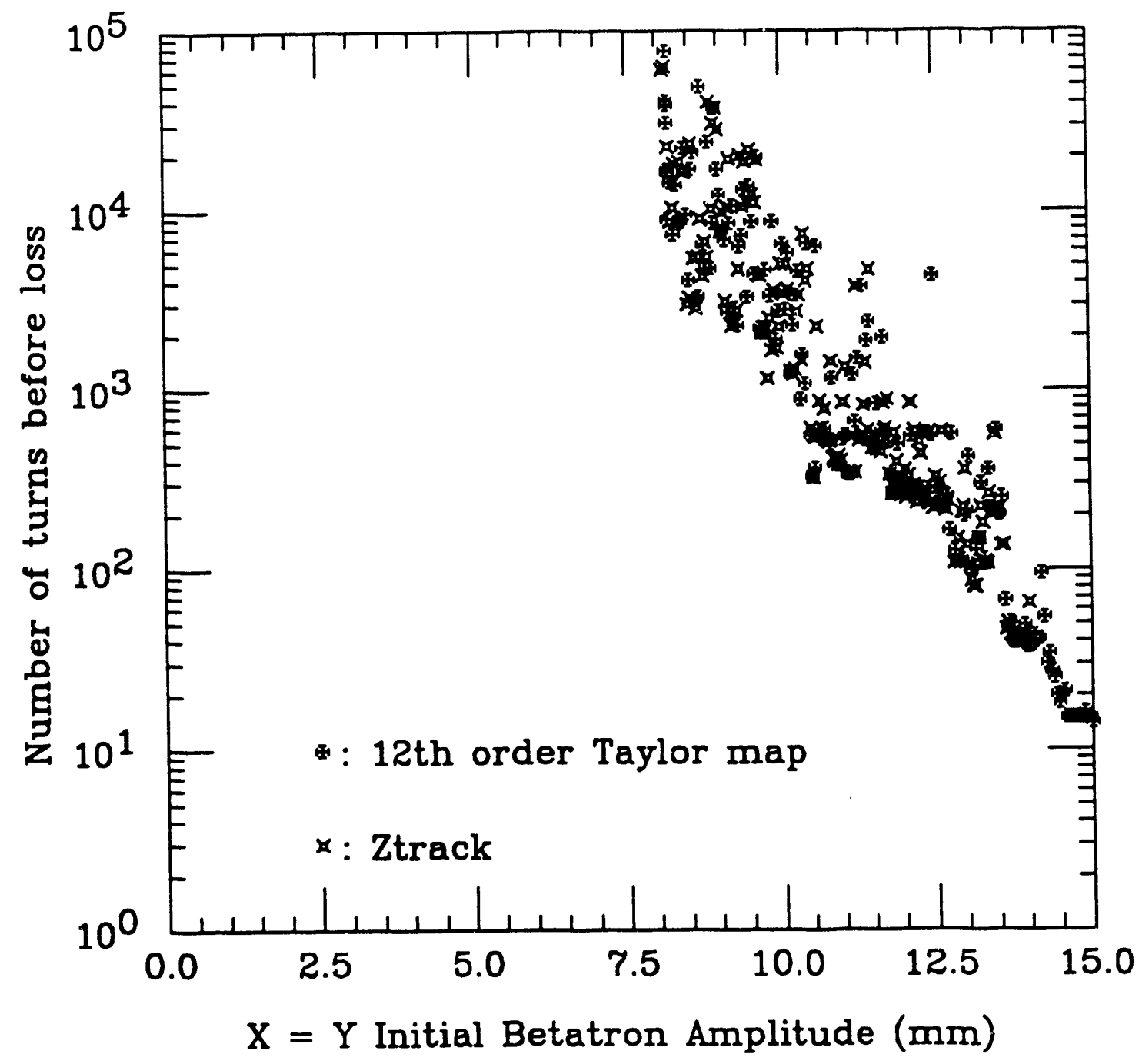

Figure 4.3. $10^{5}$-turn survival plots for a $2-\mathrm{TeV}, 5-\mathrm{cm}$-diameter dipole injection lattice of the SSC, comparing the data from a 12th-order Taylor map (extracted with Zmap) tracking with the data from its associated "Ztrack" element-byelement tracking. 
in Zlib). Shown in Figure 4.4 are two survival plots, both over one million turns; one is the extension of the 12th-order Taylor map tracking, and the other is the 11th-order Taylor map tracking. They agree with each other globally. Both show the same dynamic aperture of about $8.1 \mathrm{~mm}$ at one million turns.

Since the 4-cm-diameter dipole case has larger multipole errors and thus is more nonlinear than the 5-cm-diameter dipole case (as shown in Table 4.1), it seems that higher-order Taylor maps are necessary for the 4-cm-diameter dipole case in order to have similar studies. This is not quite true in view of the smaller dynamic aperture of the 4-cm-diameter dipole case compared to the 5-cm-diameter dipole case (as shown in Figure 4.1). The higher nonlinearity of the 4-cm-diameter dipole lattice, on one hand, results in a lower convergent rate of the power series in the Taylor map, but the reduced region of interest, on the other hand, contributes to a higher convergent rate of the power series of the map.

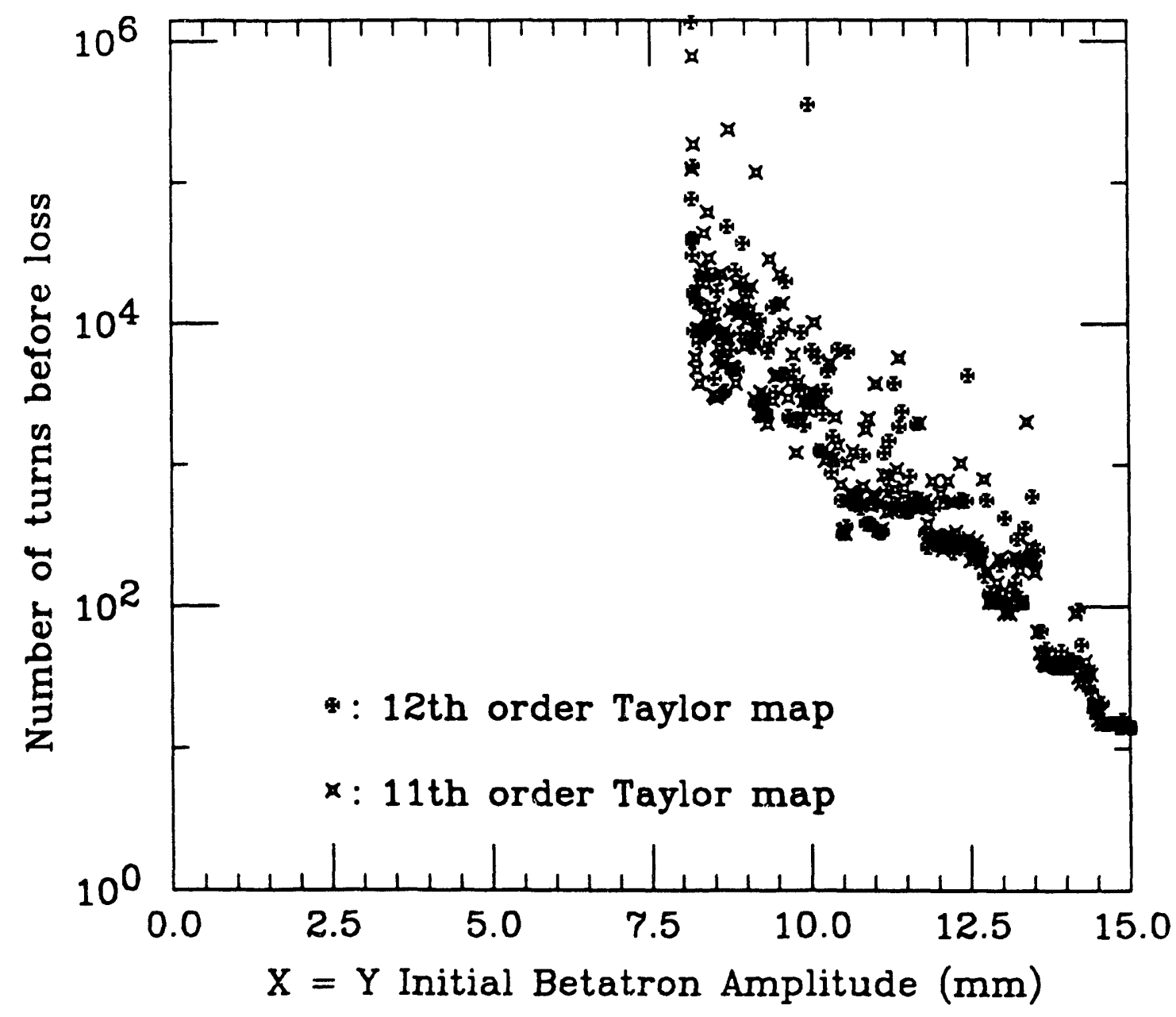

Figure 4.4. Survival plots for a $2-\mathrm{TeV}, 5$-cm-diameter dipole injection lattice of the SSC, comparing the data from the 12th-order Taylor map (extracted with Zmap) tracking with the data from the 11th-order Taylor map tracking. 
Figure 4.5 shows two survival plots, in the same frame, comparing tracking data for approximately one million turns from "Ztrack" element-by-element tracking for the 4-cm-diameter dipole SSC injection lattice and from its associated 11th-order one-turn Taylor map tracking. They also agree in global behavior, and in many details up to one million turns, as far as dynamic apertures are concerned.

For particles with small (relative to the dynamic aperture) initial transverse and longitudinal amplitudes, there is little doubt that an 11th-order or a 12thorder Taylor map tracking for the SSC is for all practical purposes as accurate as the element-by-element tracking. However, interest is in those particles with reasonable longitudinal amplitudes and with transverse amplitudes approximately

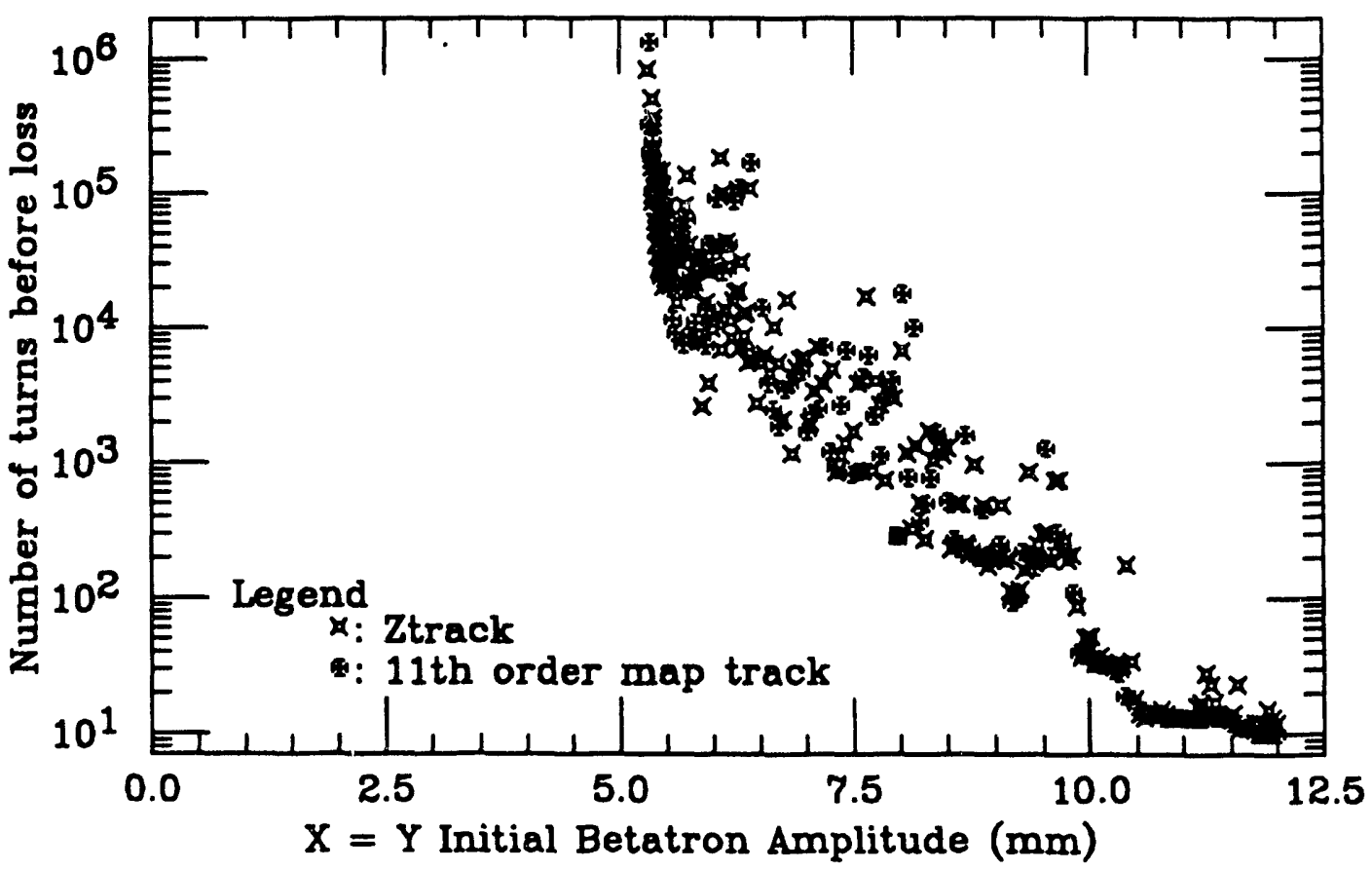

Figure 4.5. Million-turn survival plots for the 2-TeV, 4-cm-diameter dipole injection lattice of the SSC, comparing the data from an 11th-order Taylor map (extracted with "Zmap") tracking with the data from its associated "Ztrack" element-by-element tracking. The two sets of data match quite well, showing that: (a) round-off errors (64-bit precision) for long-term tracking in the SSC are of no concern since "Ztrack" element-by-element tracking is accomplished at an accuracy of 11 digits in one turn, while the 11th-order Taylor map tracking is less accurate by 3 to 4 digits ( 7 to 8 digits of accuracy) in one turn for the selected region of interest, due to truncation of higher orders, with the same result; and (b) increasing the order of the Taylor map by one or two would allow reliable fast tracking up to 10 million turns for the SSC (the required proton-coasting time of the SSC injection lattice) since increasing one order in the Taylor map will deliver nearly 10 times as much accuracy (about one more digit accuracy) in one-turn tracking. 
equal to the radius of the dynamic aperture, particularly if the ultimate goal is to determine the dynamic aperture to decide the stable region for particle motion. To be precise, Figure 4.5, for example, shows that only the 11th-order Taylor map tracking is valid up to one million turns in this case. However, considering the general property of the storage rings and the fact that increasing an order higher for the Taylor map will generally increase its one-turn-tracking accuracy by 5 to 10 times (nearly one more digit accuracy) for particles of interest (see Figure 4.6), it seems reasonable to expect that a one-turn Taylor

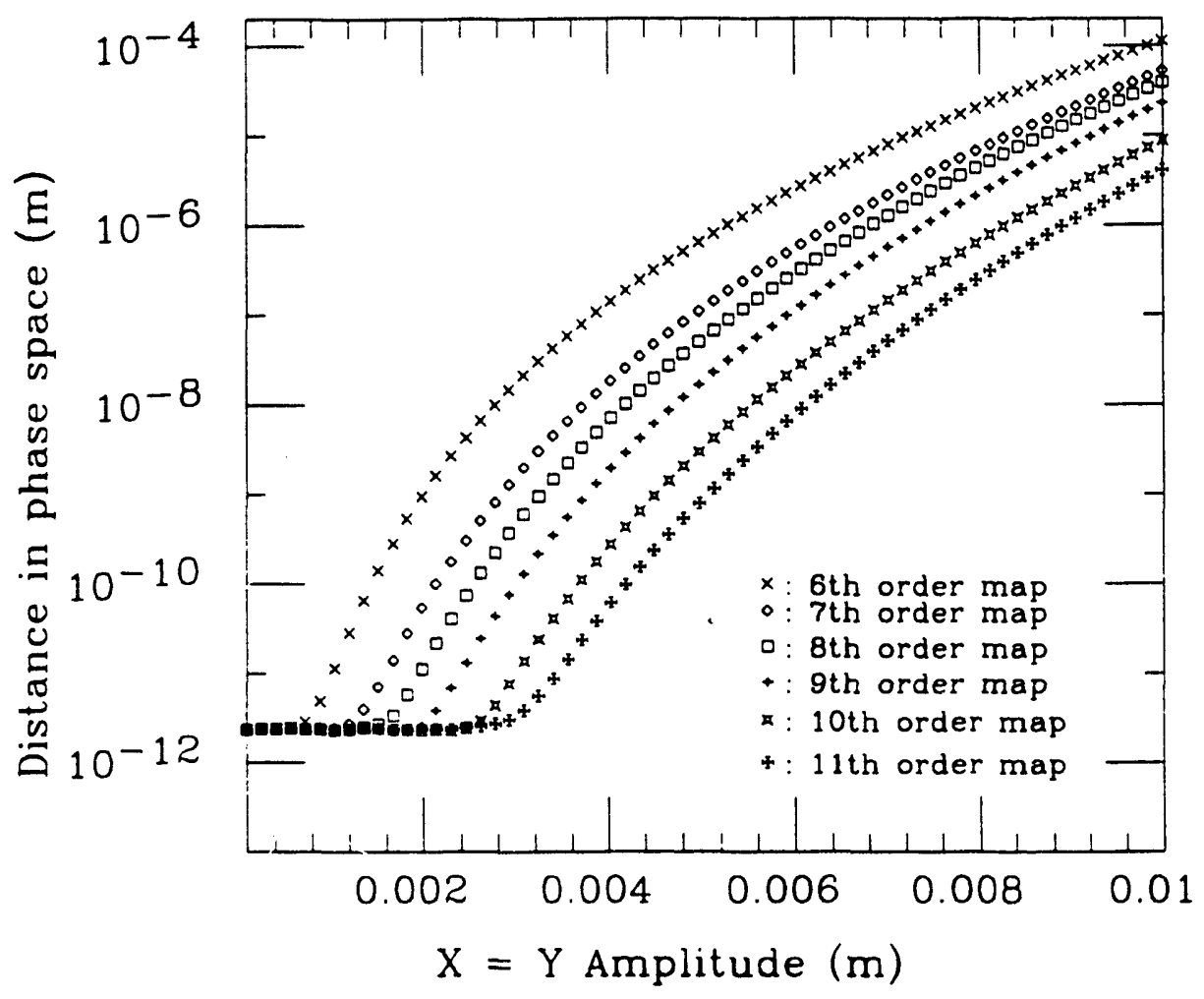

Figure 4.6. Error analysis of the one-turn Taylor map tracking for the 2-TeV, 4-cm-diameter dipole injection lattice of the SSC (the same lattice as used for Figure 4.5). The horizontal axis represents the transverse amplitude of the particles, while the vertical axis represents the weighted distance $D$ between the positions in the four-dimensional transverse phase space, obtained with the element-by-element tracking (Ztrack) and with the Taylor map tracking after one turn for each particle. $D=\left[\left(x^{Z}-x^{M}\right)^{2}+\beta_{x}^{2}\left(p_{x}^{Z}-p_{x}^{M}\right)^{2}+\left(\beta_{x} / \beta_{y}\right)\left(y^{Z}-\right.\right.$ $\left.\left.y^{M}\right)^{2}+\beta_{x} \beta_{y}\left(p_{y}^{Z}-p_{y}^{M}\right)^{2}\right]^{1 / 2}$, where $\partial \beta_{x} / \partial x=0=\partial \beta_{y} / \partial y$ at the measuring point. The deviation in energy for each of the particles is $\delta=4.2 \times 10^{-6}$. The region of interest for long-term tracking is around $0.005 \mathrm{~m}$ in transverse amplitude, where the above figure shows the accuracy of the 11th-order map to be between 7 and 8 digits after one turn. Note that for all the survival plots shown in this paper, all the particle initial energy deviations are about $\delta=5 \times 10^{-4}>4.2 \times 10^{-6}$. In such a case, the flat line at the lower left of the figure will be lifted and become longer. Nonetheless, the 7 to 8 digit accuracy at the region of interest is almost unchanged. 
map with an order less than or equal to the 14th will be valid for the SSC injection lattice lifetime ( $10^{7}$ turns) tracking. The one-turn-tracking accuracy for particles of interest is about 7 to 8 digits for the 11th-order Taylor map, due to truncation of higher orders; it is about 11 digits for the corresponding Ztrack element-by-element tracking due to round-off errors with 64-bit presision. Therefore, Figures 4.3 and 4.5 also show that the effects due to round-off errors for the SSC long-term trackings are negligible with 64-bit precision. Furthermore, round-off errors will eventually dominate the errors from the truncation of higher orders for a Taylor map tracking, if one increases the order of the map by a few orders. Such a Taylor map has almost the same degree of symplecticity compared to its corresponding symplectic tracking program from a numerical viewpoint. However, it should be repeated that a truncated Taylor map is not symplectic in general.

So far we have partially answered Question (Q-1) given in Section 4.2.2, but not Question (Q-2). Further discussions are given in the next section.

\subsubsection{Re-expanded Taylor Map Trackings}

Figure 4.7 shows survival plots, comparing the 10th-order Taylor map trackings with the corresponding element-by-element trackings for the the same lattice as for Figure 4.5. Although the 10th-order Taylor map seems adequate for

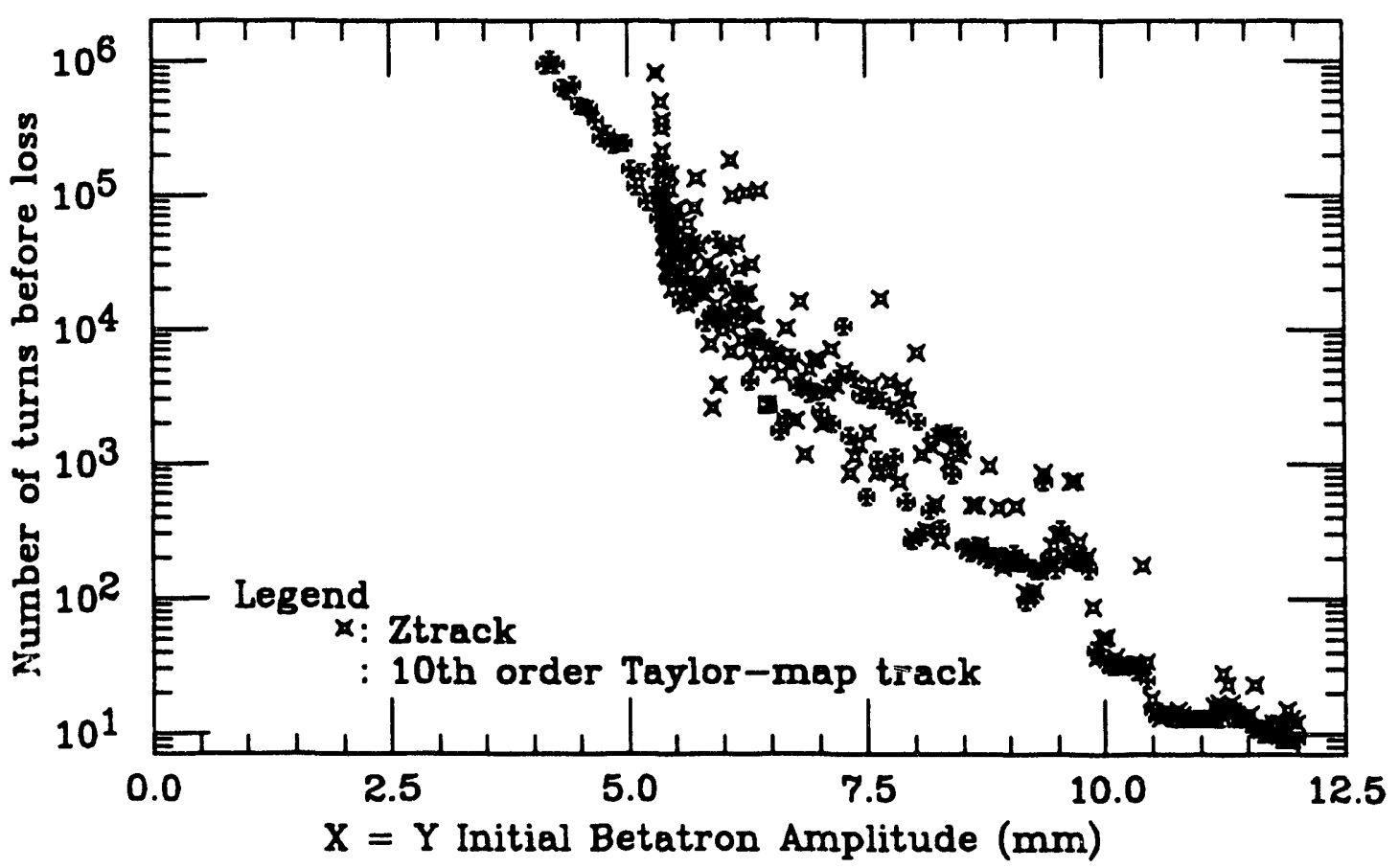

Figure 4.7. Survival plots for a 2-TeV, 4-cm-diameter dipole injection lattice of the SSC, comparing the data from the 10th-order Taylor map tracking with the data from its associated element-by-element tracking. 
tracking up to $10^{4}$ turns, it is clearly unreliable for tracking beyond $10^{5}$ turns. Without the 11th-order contents, the Taylor map is either not accurate enough or not symplectic enough, or both, for tracking up to $10^{6}$ turns. We can always improve the degree of symplecticity by transferring a truncated differential algebraic Taylor map to a series of homogeneous Lie transformations and then re-expanding the Lie transformations back to a higher-order Taylor map. (Artificial higher-order terms are added to the Taylor map to make the map more symplectic.) This has, indeed, been performed recently. ${ }^{47}$

Figure 4.8 shows survival plots, comparing the re-expanded 11th-order and the re-expanded 12th-order Taylor map trackings (re-expanded from the 10thorder) with the corresponding element-by-element trackings for the same lattice as for Figures 4.5 and 4.7. The re-expanded 12th-order case is shown in the

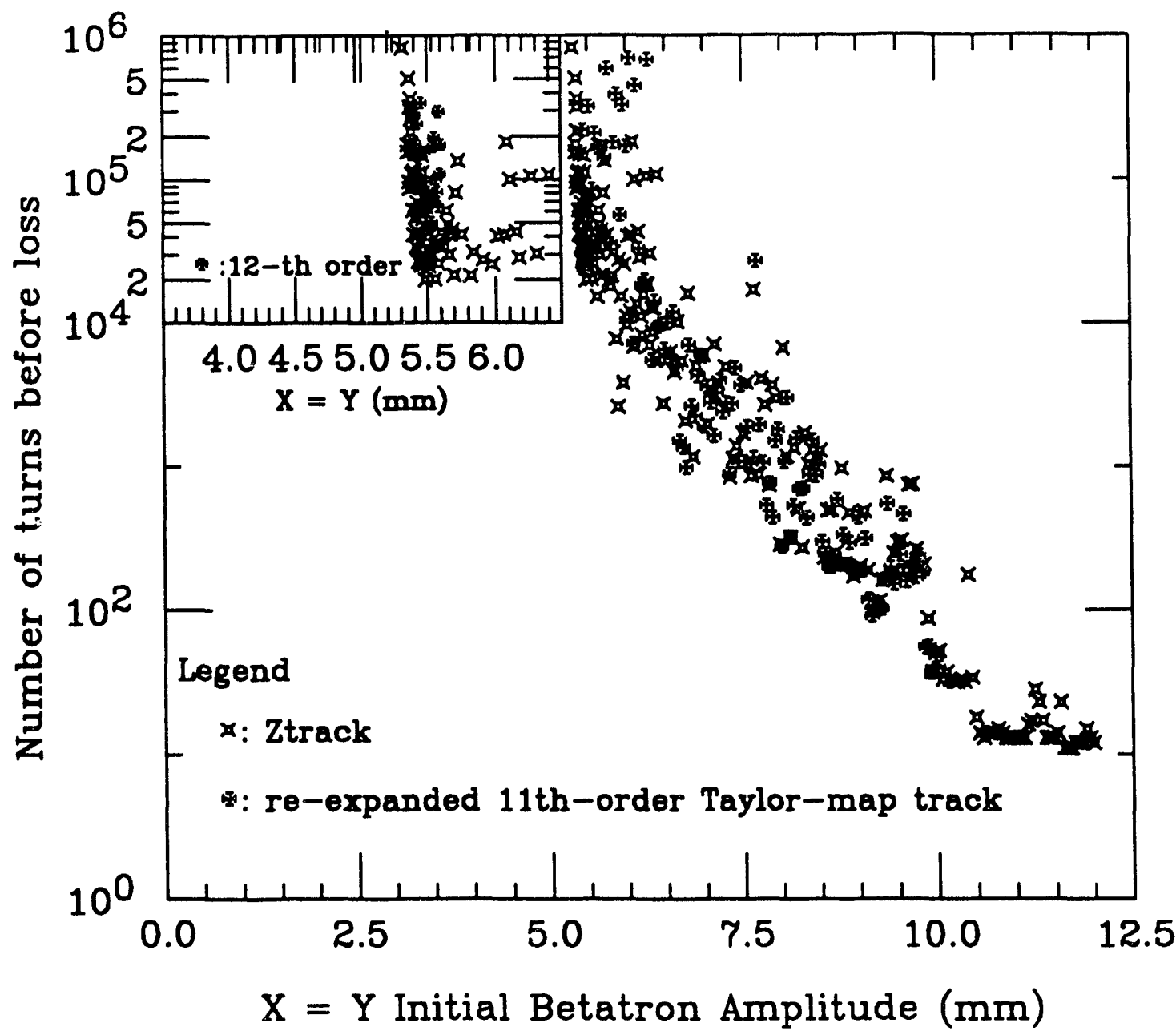

Figure 4.8. Survival plots for a $2-\mathrm{TeV}$, 4-cm-diameter dipole injection lattice of the SSC, comparing the data from the re-expanded 11th-order and the reexpanded 12th-order (left-upper corner) Taylor map trackings (re-expanded from the 10th-order) with the data from its associated element-by-element tracking. 
left-upper corner where only data near the dynamic eperture appear. The dynamic aperture for $10^{6}$ turns measured with the map has been improved to a value that is about the same as that measured with the element-by-element trackings. Further studies show that an even lower-order differential algebraic Taylor map, after improvement of symplecticity through re-expansion with its Lie transformations, can also give a reasonably good measurement of the dynamic aperture of the lattice. ${ }^{47}$

We now answer Question (Q-2) given in Section 4.2.2:

For a differential algebraic Taylor map directly extracted from a symplectic tracking program, the degree of accuracy of carrying the lattice information is higher than the degree of mathematical symplecticity. The high orders in the map are usually kept not for important lattice information but to provide the required symplecticity.

\subsubsection{A Recipe for Taylor Map Trackings}

Based on the Taylor map studies in the previous sections, a tentative recipe for long-term Taylor map trackings is given as follows.

Step 1. Extraction of a Suitably High-Order Taylor Map. For most cases, such as for the SSC, extraction of a 10th-order Taylor map should be adequate, provided that the 9th-order Taylor map has a sufficient degree of accuracy, as expected. Smaller machines can be afforded for the extraction of an even lowerorder Taylor map. Extraction of a 10th-order Taylor map for the SSC using Zmap requires about 5 hours of Cray 2 CPU time.

Step 2. Lie Transformations of the Taylor Map. Convert the Taylor map into a series of homogeneous Lie transformations up to the highest order. A 10th-order Taylor map would be converted into a series of Lie transformations up to the 11 th order. This requires only a single calling statement of the corresponding $2 \mathrm{zib}$ subroutine, using much less computer time than does the one-turn Taylor map extraction of the SSC.

Step 3. Higher-Order Taylor Map Expansions. Depending on the expected turns of tracking, the Lie transformations are converted back to two higher-order Taylor maps up to the same order. For example, the 14th-order Taylor maps would be considered for the $10^{7}$-turn (lifetime) tracking of the SSC injection lattice. The two re-expanded higher-order Taylor maps differ in that one is converted from the series of homogeneous Lie transformations up to the highest order in the series, while the other is converted from up to the second highest order in the series. In the previous example, one is converted from up to the 11th-order of the series of homogeneous Lie transformations and the other from up to the 10th-order.

Step 4. Re-expanded Taylor Map Tracking. Use the two re-expanded Taylor maps for long-term tracking up to the expected turn. Compare the survival 
plots. If the dynamic apertures from the two re-expanded Taylor map trackings match reasonably well, then we have obtained the desired results. If the dynamic apertures do not agree within a reasonable tolerance, then there are two possibilities to consider. One possibility is that the Taylor map extracted in Step 1 does not carry enough accurate information for the lattice. In this case, repeat from Step 1 for a higher-order Taylor map extraction. The other possibility is that each of the re-expanded Taylor maps contains enough information for the lattice but is still not symplectic enough for the expected long-term trackings. In this case, repeat from Step 3 for higher-order Taylor map expansions from the series of Lie transformations.

\subsubsection{Kick-Map Trackings}

Instead of re-expanding the series of Lie transformations into higher-order Taylor maps, as in Step 3 of the Taylor map tracking recipe given in the last section, one could have chosen to convert the series of Lie transformations into kick factorizations as discussed in Chapter 3. Unlike the series of Lie transformations which, although symplectic, cannot be directly used for tracking particles, the kick factorizations can be directly used for trackings and are guaranteed to be symplectic. However, the kick factorizations do not guarantee a sufficient degree of accuracy. The order of the original Taylor map must still be carefully chosen. Without parameterization of the map, one must kick-factorize the 6-dimensional map to include the longitudinal motion. In such cases, the kick-map tracking is slower than the re-expanded, suitably high-order, Taylor map tracking in general. Further discussions of the kick factorization will be given in Chapter 6 after the parameterization of the one-turn map is established.

\subsection{Multi-Turn-Map Trackings}

A one-turn map $m$ of a symplectic system can be converted into a normal form as discussed in Chapter 3. The one-turn normal form map can be written, in general, as

$$
\vec{z}^{1}=m \vec{z}=\mathcal{A}^{-1}(\vec{z})\left(\prod_{i=3}^{\Omega+1} e^{-: F_{i}(\vec{z}):}\right) e^{: h(\vec{J}):}\left(\prod_{i=3}^{\Omega+1} e^{: F_{i}(\vec{z}):}\right) \mathcal{A}(\vec{z}) \vec{z}
$$

with an accuracy up to $\Omega$ order in terms of Taylor expansions. One special feature of such a normal form map is that the $n$-turn normal form map is simply given by

$$
\vec{z}^{n}=m^{n} \vec{z}=\mathcal{A}^{-1}(\vec{z})\left(\prod_{i=3}^{\Omega+1} e^{-: F_{i}(\vec{z}):}\right) e^{: n h(\tilde{J}):}\left(\prod_{i=3}^{\Omega+1} e^{: F_{i}(\vec{z}):}\right) \mathcal{A}(\vec{z}) \vec{z} .
$$


The one-turn map can also be converted into a series of homogeneous Lie transformations via Dragt-Finn factorizations and then concatenated into a single Lie transformation via the Campbell-Baker-Hausdorff theorem. The single Lie transformation map is given, in general, by

$$
\vec{z}^{1}=m \vec{z}=e^{: f(\vec{z}):} \vec{z},
$$

where $f(\vec{z})$ is a polynomial truncated at a well-chosen order. A single Lie transformation map is similar to a normal form map in that the $n$-turn map is simply given by

$$
\bar{z}^{n}=m^{n} \vec{z}=e^{: n f(\vec{z}):} \vec{z} .
$$

How to evaluate the $n$-turn single Lie transformation map accurately and efficiently remains an open research area. Also, it should be noted that the use of the $n$-turn normal form map is limited to the phase-space area where the associated one-turn normal form map represents the original one-turn lattice with an adequate degree of accuracy. Nevertheless, evaluation of the $n$-turn normal form map takes about the same amount of computer time as that of the one-turn map. This computational time-saving feature could be particularly useful for the ongoing beam-profile evolution studies initiated by M. Syphers and currently under investigation by Kauffman et al. ${ }^{48}$

Crucial to the beam profile evolution in phase space is the transformation $m^{-n} \vec{z}$, which benefits equally from the above-mentioned time-saving feature. We now briely discuss the evolution of the beam phase space density.

Since Liouville's theorem stated that the local density is preserved, the $n$ thturn local density, as a function of the $n$ th-turn phase-space coordinates, is equal to the initial density as a function of the initial (global) phase-space coordinates; that is,

$$
\rho^{n}\left(\vec{z}^{n}\right)=\rho(\vec{z}),
$$

where $\vec{z}^{n}=m^{n} \vec{z}$ and so $\vec{z}=m^{-n} \vec{z}^{n}$. Therefore, the $n$ th-turn density profile is given by

$$
\rho^{n}(\vec{z})=\rho^{n}\left(m^{-n} \vec{z}^{n}\right)=m^{-n} \rho^{n}\left(\vec{z}^{n}\right)=m^{-n} \rho(\vec{z})=\rho\left(m^{-n} \vec{z}\right),
$$

where $m^{-n}$ is the inverse of the $n$-turn map $m^{n}$, which, for the normal form map, is given by

$$
m^{-n} \vec{z}=\mathcal{A}^{-1}(\vec{z})\left(\prod_{i=3}^{\Omega+i} e^{-: F_{i}(\vec{z}):}\right) e^{-: n h(\vec{J}):}\left(\prod_{i=3}^{\Omega+1} e^{: F_{i}(\vec{z}):}\right) \mathcal{A}(\vec{z}) \vec{z} .
$$

Currently, we are investigating the required order and the phase-space area for accurately evaluating the $n$ th-turn beam profile $\rho^{n}(\vec{z})$ at large $n$. Detailed results are to be reported. ${ }^{48}$ 


\section{Dispersed Betatron Motion}

As discussed in Chapter 2, there is always energy spread around the nominal energy in the particle beam. The energy spread causes closed-orbit spread. These dispersed closed orbits with respect to the reference orbit are functions of the longitudinal position, $s$, and of the energy deviation, $\delta=\Delta E / E_{0}$ (or the off-momentum, $\left.\delta=\Delta p / p_{0}\right)$; that is,

$$
\vec{x}_{c}=\vec{x}_{c}(\delta, s)
$$

where $\vec{x}_{c}$ is a vector representing the transverse phase-space coordinates of the dispersed closed orbit, and its transpose is given by

$$
\vec{x}_{c}^{T}=\left(x_{c}, p_{x, c}, y_{c}, p_{y, c}\right)
$$

As the particles traverse around, they perform betatron oscillations with respect to their dispersed closed orbit.

In this chapter, we shall not discuss synchrotron oscillations. We shall assume the presence of either no RF cavity in the storage ring or of a single RF cavity with a tracking model that treats the RF cavity as a thin RF kick for the longitudinal momentum between two drifts. In this way the thin RF kick can be separately treated from the one-turn transverse map by choosing the longitudinal reference position of the map at the position where the thin RF kick is located. At a fixed longitudinal reference position, the dependence of the dispersed closed orbits on the longitudinal position, $s$, can be dropped out of the mathematical formula; thus we have

$$
\vec{x}_{c}=\vec{x}_{c}(\delta)
$$

An exact formula for the dispersed closed orbit as a function of the energy deviation, $\delta$, is not obtainable generally. However, with a differential algebraic map extraction program such as $\mathrm{Zmap}$, the dispersed closed orbit can be obtained as a polynomial of the energy deviation, $\delta$, up to a specified order given by

$$
\vec{x}_{c}(\delta)=\vec{x}_{c, 0}+\delta \vec{x}_{c, 1}+\delta^{2} \vec{x}_{c, 2}+\cdots
$$

A one-turn Taylor map can then be extracted with respect to the dispersed closed orbit and given by

$$
\vec{x}^{\prime}=m(\vec{x}, \delta) \vec{x}=\vec{U}(\vec{x}, \delta)=M(\delta) \vec{x}+\vec{U}_{2}(\vec{x}, \delta)+\vec{U}_{3}(\vec{x}, \delta)+\cdots
$$

where the transverse canonical phase-space coordinates $\vec{x}$, given by $\vec{x}^{T}=$ $\left(x, p_{x}, y, p_{y}\right)$, are deviations relative to the dispersed closed orbit $\vec{x}_{c}(\delta) ; \delta$ is 
a parameter for the energy deviation, which is usually a smallness factor in the domain of physical interest; $\vec{U}_{k}(\vec{x}, \delta)$ for $k=2,3, \cdots$ are homogeneous vector polynomials of degree $k$ in $\vec{x}$; and $M(\delta)$ is the parameterized Courant-Snyder matrix that can be reformulated as

$$
M(\delta)=M_{0}+\delta M_{1}+\delta^{2} M_{2}+\cdots,
$$

where $M_{0}, M_{1}, M_{2}, \cdots$ are $4 \times 4$ matrices.

We shall further confine ourselves in this chapter to the study of the parameterized Courant-Snyder matrix $M(\delta)$ given by Eq. (5.3). The general parameterized map given by Eq. (5.2) is to be studied in the next chapter.

\subsection{The Parameterized Courant-Snyder Matrix}

If we neglect the nonlinear multipole error effects, a one-turn map with respect to the dispersed closed orbit would be simply a parameterized CourantSnyder map given by

$$
\vec{x}=M(\delta) \vec{x},
$$

where $M(\delta)$ is given by Eq. (5.3). The matrix $M_{0}$ is symplectic, but $M(\delta)$ generally is not unless it is expanded to the infinite order of $\delta$. However, $M(\delta)$ preserves one important symplectic property of the system, which is

$$
M^{T}(\delta) S M(\delta)=S+\sigma\left(\delta^{n+1}\right),
$$

where $n$ is the truncated order of $M(\delta)$, and $S$ is the symplectic identity discussed in Chapter 3.

Since an infinite order $M(\delta)$ is not obtainable, $M(\delta)$ is used for convenience with an implicit understanding that it represents a parameterized CourantSnyder matrix truncated at a finite order $\delta^{n}$; that is,

$$
M(\delta)=M_{0}+\delta M_{1}+\delta^{2} M_{2}+\cdots+\delta^{n} M_{n}
$$

Occasionally, $M(\delta)$ will also be referred to as a symplectic parameterized matrix up to the order of $\delta^{n}$, although the terminology may not be strictly correct.

\subsection{Uncoupled Dispersed Betatron Motion}

For simplicity and in particular for initial lattice design, we shall start with the uncoupled case; that is, we shall assume that the two degrees of freedom of the horizontal and vertical motion are independent. Thus, the matrices in Eq. (5.4) can be considered as $2 \times 2$ matrices. The main task of this section is 
to make a series of canonical transformations such that a parameterized normal form can be obtained as follows:

$$
R_{n}(\delta)=\left(\begin{array}{cc}
\cos \mu(\delta) & \sin \mu(\delta) \\
-\sin \mu(\delta) & \cos \mu(\delta)
\end{array}\right)=A^{-1}(\delta) M(\delta) A(\delta)+\sigma\left(\delta^{n+1}\right)
$$

Neglecting $\sigma\left(\delta^{n+1}\right)$, a truncated parameterized Courant-Snyder Matrix $M(\delta)$ can be represented by a symplectic matrix given by

$$
\begin{aligned}
M_{s}(\delta) & =A(\delta) R_{n}(\delta) A^{-1}(\delta) \\
& =\left(\begin{array}{cc}
\cos \mu(\delta)+\alpha(\delta) \sin \mu(\delta) & \beta(\delta) \sin \mu(\delta) \\
-\gamma(\delta) \sin \mu(\delta) & \cos \mu(\delta)-\alpha(\delta) \sin \mu(\delta)
\end{array}\right),
\end{aligned}
$$

where the series of canonical generation matrices have been concatenated into a single symplectic matrix $\mathrm{A}(\delta)$ of order $\delta^{n(n+1) / 2}$, and the parameterized tune $\mu(\delta)$ of order $\delta^{n}$ and Twiss parameters $\beta(\delta), \alpha(\delta)$, and $\gamma(\delta)$ of orders $\delta^{n(n+1)}$ can be explicitly calculated as power series expansions of $\delta$ through the order-byorder normalization of the parameterized Courant-Snyder matrix.

\subsubsection{Normalization of the Oth-Order Courant-Snyder Matrix}

We begin with the normalization of the 0th-order Courant-Snyder matrix, and we will consider stable motion only. The main task is to find a symplectic generation matrix $A_{0}$ and its inverse $A_{0}^{-1}$ (also symplectic) such that

$$
R_{0}=\left(\begin{array}{cc}
\cos \mu_{0} & \sin \mu_{0} \\
-\sin \mu_{0} & \cos \mu_{0}
\end{array}\right)=A_{0}^{-1} M_{0} A_{0}
$$

This has been well understood since the proposal of the alternating-gradient synchrotron. ${ }^{1}$ Let

$$
M_{0}=\left(\begin{array}{ll}
a_{0} & b_{0} \\
c_{0} & d_{0}
\end{array}\right),
$$

with (a) $a_{0} d_{0}-b_{0} c_{0}=1$ for $M_{0}$ to be symplectic, and (b) $\left|\frac{1}{2}\left(a_{0}+d_{0}\right)\right|<1$ and $b_{0} c_{0}<0$ for stable motion. Then

$$
\cos \mu_{0}=\frac{1}{2} \operatorname{Tr}\left(M_{0}\right)=\frac{1}{2}\left(a_{0}+d_{0}\right) .
$$

With a unique solution for $\mu_{0}$ by choosing

$$
\sin \mu_{0}=-\frac{c_{0}}{\left|c_{0}\right|} \sqrt{1-\cos ^{2} \mu_{0}}
$$


the generation matrix $A_{0}$ is generally given by

$$
A_{0}=\left(\begin{array}{cc}
\frac{y\left(a_{0}-\cos \mu_{0}\right)-x \sin \mu_{0}}{c_{0} \sqrt{\beta_{0}}} & \frac{x\left(a_{0}-\cos \mu_{0}\right)+y \sin \mu_{0}}{c_{0} \sqrt{\beta_{0}}} \\
\frac{y}{\sqrt{\beta_{0}}} & \frac{x}{\sqrt{\beta_{0}}}
\end{array}\right),
$$

where

$$
\beta_{0}=-\frac{1}{c_{0}}\left(x^{2}+y^{2}\right) \sin \mu_{0}>0
$$

and $x$ and $y$ can be any real numbers. By choosing $x=1$ and $y=-\left(a_{0}-\right.$ $\left.\cos \mu_{0}\right) /\left(\sin \mu_{0}\right)$, we have the Courant-Snyder's choice that

$$
A_{0}=\left(\begin{array}{cc}
\sqrt{\beta_{0}} & 0 \\
\frac{-\alpha_{0}}{\sqrt{\beta_{0}}} & \frac{1}{\sqrt{\beta_{0}}}
\end{array}\right), \quad A_{0}^{-1}=\left(\begin{array}{cc}
\frac{1}{\sqrt{\beta_{0}}} & 0 \\
\frac{\alpha_{0}}{\sqrt{\beta_{0}}} & \sqrt{\beta_{0}}
\end{array}\right),
$$

where

$$
\beta_{0}=\frac{b_{0}}{\sin \mu_{0}}, \quad \alpha_{0}=\frac{a_{0}-d_{0}}{2 \sin \mu_{0}}
$$

$M_{0}$ is then given by

$$
M_{0}=\hat{A}_{\bar{u}} D_{0} A_{0}^{-1}=\left(\begin{array}{cc}
\cos \mu_{0}+\alpha_{0} \sin \mu_{0} & \beta_{0} \sin \mu_{0} \\
-\gamma_{0} \sin \mu_{0} & \cos \mu_{0}-\alpha_{0} \sin \mu_{0}
\end{array}\right) \text {, }
$$

where

$$
\gamma_{0}=\frac{1+\alpha_{0}^{2}}{\beta_{0}}
$$

The above solution can be easily checked by substituting $A_{0}$ and $M_{0}$ into Eq. (5.6).

\subsubsection{Normalization of the 1st-Order Courant-Snyder Matrix}

We now proceed to the normalization of the 1st-order Courant-Snyder matrix by first making a canonical transformation of $M(\delta)$ as follows:

$$
\begin{aligned}
{ }_{1} M(\delta)=A_{0}^{-1} M(\delta) A_{0} & =A_{0}^{-1} M_{0} A_{0}+\delta A_{0}^{-1} M_{1} A_{0}+\cdots+\delta^{n} A_{0}^{-1} M_{n} A_{0}+\sigma\left(\delta^{n+1}\right) \\
& =R_{0}+\delta_{1} M_{1}+\delta^{2}{ }_{1} M_{2}+\cdots+\delta^{n}{ }_{1} M_{n}+\sigma\left(\delta^{n+1}\right) .
\end{aligned}
$$

Since $A_{0}$ and $A_{0}^{-1}$ are symplectic, similar to $M(\delta),{ }_{1} M(\delta)$ has the following property:

$$
{ }_{1} M^{T}(\delta) S{ }_{1} M(\delta)=S+\sigma\left(\delta^{n+1}\right)
$$


This, together with $R_{0}$ being symplectic, means that $\left(R_{0}^{T} S R_{0}=S\right)$ yields the following necessary conditions:

$$
\begin{aligned}
& \delta^{1}: R_{0}^{T} S{ }_{1} M_{1}+{ }_{1} M_{1}^{T} S R_{0}=0 \\
& \quad \vdots \\
& \delta^{n}: R_{0}^{T} S_{1} M_{n}+{ }_{1} M_{n}^{T} S R_{0}+\cdots+{ }_{1} \bar{S}_{n}=0
\end{aligned}
$$

where

$$
\begin{cases}{ }_{1} \bar{S}_{n}={ }_{1} M_{n / 2}^{T} S_{1} M_{n / 2} & \text { if } n \text { is even } \\ { }_{1} \bar{S}_{n}={ }_{1} M_{(n-1) / 2}^{T} S_{1} M_{(n+1) / 2}+{ }_{1} M_{(n+1) / 2}^{T} S_{1} M_{(n-1) / 2} & \text { if } n \text { is odd }\end{cases}
$$

The main task, then, is to find a $\delta$-dependent symplectic generation matrix $\bar{A}_{1}(\delta)=I+\delta A_{1}$ such that

$R_{1}(\delta)=\left(\begin{array}{cc}\cos \left(\mu_{0}+\delta \mu_{1}\right) & \sin \left(\mu_{0}+\delta \mu_{1}\right) \\ -\sin \left(\mu_{0}+\delta \mu_{1}\right) & \cos \left(\mu_{0}+\delta \mu_{1}\right)\end{array}\right)=\bar{A}_{1}^{-1}(\delta)\left(R_{0}+\delta_{1} M_{1}\right) \bar{A}_{1}(\delta)+\sigma\left(\delta^{2}\right)$, where $I=\left(\begin{array}{ll}1 & 0 \\ 0 & 1\end{array}\right)$ is the identity matrix and $A_{1}$ is independent of $\delta$.

There are two necessary conditions for $\bar{A}_{1}(\delta)$ to be symplectic:

$$
A_{1}^{T} S+S A_{1}=0 \Rightarrow \operatorname{Tr}\left(A_{1}\right)=0
$$

and

$$
A_{1}^{T} S A_{1}=0 \Rightarrow \operatorname{det}\left(A_{1}\right)=0 \text {. }
$$

Thus $A_{1} A_{1}=0$; that is, $A_{1}$ is a nil-potent matrix. Therefore, the inverse matrix of $\bar{A}_{1}(\delta)$ is simply

$$
\bar{A}_{1}^{-1}(\delta)=I-\delta A_{1}
$$


which is also symplectic. Let

$$
{ }_{1} M_{1}=\left(\begin{array}{ll}
a_{1} & b_{1} \\
c_{1} & d_{1}
\end{array}\right)
$$

with a constraint that $\left(a_{1}+d_{1}\right) \cos \mu_{0}=\left(c_{1}-b_{1}\right) \sin \mu_{0}$, since $R_{0}^{T} S{ }_{1} M_{1}+$ ${ }_{1} M_{1}^{T} S R_{0}=0$. Expanding Eq. (5.8) up to order $\delta$, we obtain

$$
\delta \mu_{1}\left(\begin{array}{cc}
-\sin \mu_{0} & \cos \mu_{0} \\
-\cos \mu_{0} & -\sin \mu_{0}
\end{array}\right)=\delta\left(R_{0} A_{1}-A_{1} R_{0}+{ }_{1} M_{1}\right)+\sigma\left(\delta^{2}\right)
$$

that is,

$$
\mu_{1}\left(\begin{array}{cc}
-\sin \mu_{0} & \cos \mu_{0} \\
-\cos \mu_{0} & -\sin \mu_{0}
\end{array}\right)=R_{0} A_{1}-A_{1} R_{0}+{ }_{1} M_{1} .
$$

With the given constraints on $A_{1}: \operatorname{Tr}\left(A_{1}\right)=0$ and $\operatorname{det}\left(A_{1}\right)=0$ and on ${ }_{1} M_{1}$ : $\left(a_{1}+d_{1}\right) \cos \mu_{0}=\left(c_{1}-b_{1}\right) \sin \mu_{0}$, we obtain

$$
\mu_{1}=-\frac{\operatorname{Tr}\left({ }_{1} M_{1}\right)}{2 \sin \mu_{0}}=-\frac{a_{1}+d_{1}}{2 \sin \mu_{0}}
$$

and

$$
A_{1}=\frac{1}{4 \sin \mu_{0}}\left(\begin{array}{cc}
b_{1}+c_{1} & d_{1}-a_{1} \pm \sqrt{h_{1}} \\
d_{1}-a_{1} \mp \sqrt{h_{1}} & -\left(b_{1}+c_{1}\right)
\end{array}\right),
$$

where

$$
h_{1}=\left(a_{1}-d_{1}\right)^{2}+\left(b_{1}+c_{1}\right)^{2}=\left[\frac{\operatorname{Tr}\left({ }_{1} M_{1}\right)}{\sin \mu_{0}}\right]^{2}-4 \operatorname{det}\left({ }_{1} M_{1}\right) \text {. }
$$

The above solutions can be easily checked by substituting $\bar{A}_{1}=I+\delta A_{1}$ and ${ }_{1} M_{1}$ into Eq. (5.8). The constraint that $A_{1}$ is a nil-potent matrix can also be easily checked. Note that $\mu_{1}$ as given by Eq. (5.10) is the "chromaticity" discussed in Chapter 2.

\subsubsection{Normalization up to the nth-Order Courant-Snyder Matrix}

A process similar to that used in the last section for the normalization of the 1st-order Courant-Snyder Matrix can be used for higher-order normalizations. ${ }^{49}$ 
Let us assume that an $(i-1)$ th-order generation matrix $\bar{A}_{i-1}(\delta)=I+$ $\delta^{i-1} A_{i-1}$ has been obtained, and that a canonical transformation has been made such that

$$
\begin{aligned}
{ }_{i} M(\delta) & =\bar{A}_{i-1}^{-1}(\delta)_{i-1} M(\delta) \bar{A}_{i-1}(\delta) \\
& =R_{i-1}(\delta)+\delta^{i}{ }_{i} M_{i}+\delta^{i+1}{ }_{i} M_{i+1}+\cdots+\delta^{n}{ }_{i} M_{n}+\sigma\left(\delta^{n+1}\right)
\end{aligned}
$$

where $R_{i-1}(\delta)$ is a rotation given by

$R_{i-1}(\delta)=\left(\begin{array}{cc}\cos \left(\mu_{0}+\delta \mu_{1}+\cdots+\delta^{i-1} \mu_{i-1}\right) & \sin \left(\mu_{0}+\delta \mu_{1}+\cdots+\delta^{i-1} \mu_{i-1}\right) \\ -\sin \left(\mu_{0}+\delta \mu_{1}+\cdots+\delta^{i-1} \mu_{i-1}\right) & \cos \left(\mu_{0}+\delta \mu_{1}+\cdots+\delta^{i-1} \mu_{i-1}\right)\end{array}\right)$

and

$$
{ }_{i} M_{i}=\left(\begin{array}{ll}
a_{i} & b_{i} \\
c_{i} & d_{i}
\end{array}\right)
$$

with a constraint that $\left(a_{i}+d_{i}\right) \cos \mu_{0}=\left(c_{i}-b_{i}\right) \sin \mu_{0}$, which is one of the necessary conditions from the property that

$$
{ }_{i} M^{T}(\delta) S,{ }_{i} M(\delta)=S+\sigma\left(\delta^{n+1}\right) .
$$

The $i$ th order symplectic generation matrix $\bar{A}_{i}(\delta)$ is then given by

$$
\bar{A}_{i}(\delta)=I+\delta^{i} A_{i}
$$

where

$$
A_{i}=\frac{1}{4 \sin \mu_{0}}\left(\begin{array}{cc}
b_{i}+c_{i} & d_{i}-a_{i} \pm \sqrt{h_{i}} \\
d_{i}-a_{i} \mp \sqrt{h_{i}} & -\left(b_{i}+c_{i}\right)
\end{array}\right)
$$

and

$$
h_{i}=\left(a_{i}-d_{i}\right)^{2}+\left(b_{i}+c_{i}\right)^{2}=\left[\frac{\operatorname{Tr}\left({ }_{i} M_{i}\right)}{\sin \mu_{0}}\right]^{2}-4 \operatorname{det}\left({ }_{i} M_{i}\right) .
$$

A canonical transformation can then be made to obtain ${ }_{i+1} M(\delta)$ as follows:

$$
\begin{aligned}
& { }_{i+1} M(\delta)=\left(I-\delta^{i} A_{i}\right)_{i} M(\delta)\left(I+\delta^{i} A_{i}\right) \\
& =\left(I-\delta^{i} A_{i}\right)\left(R_{i-1}(\delta)+\delta^{i}{ }_{i} M_{i}\right)\left(I+\delta^{i} A_{i}\right) \\
& +\delta^{i+1}\left(I-\delta^{i} A_{i}\right)_{i} M_{i+1}\left(I+\delta^{i} A_{i}\right) \\
& +\cdots+\delta^{n}\left(I-\delta^{i} A_{i}\right)_{i} M_{n}\left(I+\delta^{i} A_{i}\right)+\sigma\left(\delta^{n+1}\right) \\
& =R_{i}(\delta)+\delta^{i+1}{ }_{i+1} M_{i+1}+\delta^{i+2}{ }_{i+1} M_{i+2}+\cdots+\delta^{n}{ }_{i+1} M_{n}+\sigma\left(\delta^{n+1}\right) \text {, }
\end{aligned}
$$


where the rotation $R_{i}(\delta)$ is given by

$$
R_{i}(\delta)=\left(\begin{array}{cc}
\cos \left(\mu_{0}+\delta \mu_{1}+\cdots+\delta^{i} \mu_{i}\right) & \sin \left(\mu_{0}+\delta \mu_{1}+\cdots+\delta^{i} \mu_{i}\right) \\
-\sin \left(\mu_{0}+\delta \mu_{1}+\cdots+\delta^{i} \mu_{i}\right) & \cos \left(\mu_{0}+\delta \mu_{1}+\cdots+\delta^{i} \mu_{i}\right)
\end{array}\right),
$$

and

$$
\mu_{i}=-\frac{\operatorname{Tr}\left({ }_{i} M_{i}\right)}{2 \sin \mu_{0}}=-\frac{a_{i}+d_{i}}{2 \sin \mu_{0}} .
$$

The above process is then iterated until we obtain the $n$ th-order symplectic generation matrix $\bar{A}_{n}(\delta)=I+\delta^{n} A_{n}$ and then make the $(n+1)$ th canonical transformation to obtain

$$
\left(I-\delta^{n} A_{n}\right)_{n} M(\delta)\left(I+\delta^{n} A_{n}\right)=R_{n}(\delta)+\sigma\left(\delta^{n+1}\right) .
$$

Tracing back the $n+1$ canonical transformations, we have $M(\delta)=A_{0}\left(I+\delta A_{1}\right) \cdots\left(I+\delta^{n} A_{n}\right) R_{n}(\delta)\left(I-\delta^{n} A_{n}\right) \cdots\left(I-\delta A_{1}\right) A_{0}^{-1}+\sigma\left(\delta^{n+1}\right)$, where

$$
R_{n}(\delta)=\left(\begin{array}{cc}
\cos \mu(\delta) & \sin \mu(\delta) \\
-\sin \mu(\delta) & \cos \mu(\delta)
\end{array}\right)
$$

is a rotation (a rotation is always symplectic) and $\mu(\delta)=\mu_{0}+\delta \mu_{1}+\delta^{2} \mu_{2}+\cdots+$ $\delta^{n} \mu_{n} ; A_{0}$ and its inverse $A_{0}^{-1}$ are the usual Courant-Snyder generation matrix obtained from $M_{0}$ (note: $M_{0}^{T} S M_{0}=S$ ); and $\left(I+\delta^{i} A_{i}\right.$ ) and their inverses $\left(I-\delta^{i} A_{i}\right)$ for $i=1,2, \ldots, n$, are the order-by-order generation matrices, which are all symplectic.

In a practical case, $\sigma\left(\delta^{n+1}\right)$ is usually negligible if $\delta$ is small and $n$ is large enough, so that $M(\delta)$ can be represented by the symplectic matrix

$$
M_{s}(\delta)=A_{0}\left(I+\delta A_{1}\right) \cdots\left(I+\delta^{n} A_{n}\right) R_{n}(\delta)\left(I-\delta^{n} A_{n}\right) \cdots\left(I-\delta A_{1}\right) A_{0}^{-1} .
$$

\subsubsection{The Parameterized Twiss Parameters}

The symplectic matrix $M_{s}(\delta)$ given by Eq. (5.13) can be converted into the Twiss formulation given by Eq. (5.5). The parameterized Twiss parameters can be obtained in the forms of finite-order power series expansions of $\delta$ given by

$$
\begin{aligned}
& \beta(\delta)=\beta_{0}+\delta \beta_{1}+\delta^{2} \beta_{2}+\cdots+\delta^{n} \beta_{n}+\cdots+\delta^{n(n+1)} \beta_{n(n+1)}, \\
& \gamma(\delta)=\gamma_{0}+\delta \gamma_{1}+\delta^{2} \gamma_{2}+\cdots+\delta^{n} \gamma_{n}+\cdots+\delta^{n(n+1)} \gamma_{n(n+1)}, \\
& \alpha(\delta)=\alpha_{0}+\delta \alpha_{1}+\delta^{2} \alpha_{2}+\cdots+\delta^{n} \alpha_{n}+\cdots+\delta^{n(n+1)} \alpha_{n(n+1)},
\end{aligned}
$$

such that the Twiss condition, $1+\alpha^{2}(\delta)=\beta(\delta) \gamma(\delta)$, is satisfied exactly. 
To obtain the parameterized Twiss parameters, $\beta(\delta), \gamma(\delta)$, and $\alpha(\delta)$ explicitly, that is, to obtain $\beta_{i}, \gamma_{i}$, and $\alpha_{i}$ for $i=1,2, \cdots, n(n+1)$, one first expands Eq. (5.5) and Eq. (5.13) while keeping intact $\cos \mu(\delta)$ and $\sin \mu(\delta)$ as follows:

$$
\begin{aligned}
M_{s}(\delta)= & \left(\begin{array}{cc}
\cos \mu(\delta)+\alpha(\delta) \sin \mu(\delta) & \beta(\delta) \sin \mu(\delta) \\
-\gamma(\delta) \sin \mu(\delta) & \cos \mu(\delta)-\alpha(\delta) \sin \mu(\delta)
\end{array}\right) \\
= & \left(\begin{array}{cc}
\cos \mu(\delta)+\alpha_{0} \sin \mu(\delta) & \beta_{0} \sin \mu(\delta) \\
-\gamma_{0} \sin \mu(\delta) & \cos \mu(\delta)-\alpha_{0} \sin \mu(\delta)
\end{array}\right) \\
& +\delta \sin \mu(\delta)\left(\begin{array}{cc}
\alpha_{1} & \beta_{1} \\
-\gamma_{1} & -\alpha_{1}
\end{array}\right)+\delta^{2} \sin \mu(\delta)\left(\begin{array}{cc}
\alpha_{2} & \beta_{2} \\
-\gamma_{2} & -\alpha_{2}
\end{array}\right)+\cdots \\
& +\delta^{n(n+1)} \sin \mu(\delta)\left(\begin{array}{cc}
\alpha_{n(n+1)} & \beta_{n(n+1)} \\
-\gamma_{n(n+1)} & -\alpha_{n(n+1)}
\end{array}\right)
\end{aligned}
$$

and

$$
\begin{aligned}
M_{s}(\delta)= & A_{0} R_{n}(\delta) A_{0}^{-1}+\delta A_{0}\left[A_{1} R_{n}(\delta)-R_{n}(\delta) A_{1}\right] A_{0}^{-1} \\
& +\delta^{2} A_{0}\left[A_{2} R_{n}(\delta)-R_{n}(\delta) A_{2}-A_{1} R_{n}(\delta) A_{1}\right] A_{0}^{-1}+\cdots
\end{aligned}
$$

One then substitutes Eq. (5.7) for $A_{0}$ and $A_{0}^{-1}$ and Eq. (5.12) for $A_{i}, i=$ $1,2, \cdots, n$, into Eq. (5.15), then compares it with Eq. (5.14). The first-order and the second-order perturbations of Twiss parameters are given as follows:

$$
\begin{aligned}
\beta_{1}= & \frac{\beta_{0}\left(b_{1}+c_{1}\right)}{2 \sin \mu_{0}} \\
\alpha_{1}= & \frac{\alpha_{0}\left(b_{1}+c_{1}\right)+\left(a_{1}-d_{1}\right)}{2 \sin \mu_{0}} \\
\gamma_{1}= & \frac{\alpha_{0}^{2}\left(b_{1}+c_{1}\right)+2 \alpha_{0}\left(a_{1}-d_{1}\right)-\left(b_{1}+c_{1}\right)}{2 \beta_{0} \sin \mu_{0}} \\
\beta_{2}= & \frac{\beta_{0}\left(b_{2}+c_{2}\right)}{2 \sin \mu_{0}}+\frac{\beta_{0}\left[h_{1} \mp\left(a_{1}-d_{1}\right) \sqrt{h_{1}}\right]}{8 \sin \mu_{0}}, \\
\alpha_{2}= & \frac{\alpha_{0}\left(b_{2}+c_{2}\right)+\left(a_{2}-d_{2}\right)}{2 \sin \mu_{0}}+\frac{ \pm\left(b_{1}+c_{1}\right) \sqrt{h_{1}}+\alpha_{0} h_{1} \mp \alpha_{0}\left(a_{1}-d_{1}\right) \sqrt{h_{1}}}{8 \sin \mu_{0}} \\
\gamma_{2}= & \frac{\alpha_{0}^{2}\left(b_{2}+c_{2}\right)+2 \alpha_{0}\left(a_{2}-d_{2}\right)-\left(b_{2}+c_{2}\right)}{2 \beta_{0} \sin \mu_{0}} \\
& +\frac{\left(1+\alpha_{0}^{2}\right) h_{1} \pm\left(1-\alpha_{0}^{2}\right)\left(a_{1}-d_{1}\right) \sqrt{h_{1}} \pm 2 \alpha_{0}\left(b_{1}+c_{1}\right) \sqrt{h_{1}}}{8 \sin \mu_{0}} .
\end{aligned}
$$


Note that $a_{i}=b_{i}=c_{i}=d_{i}=h_{i}=0$ for $i>n$. Note also that these uncoupled order-by-order Twiss parameters can be obtained up to the truncated order $\delta^{n}$ (but not up to the symplectified order $\delta^{n(n+1)}$ ) by a direct series expansion of the $2 \times 2$ parameterized Courant-Snyder matrix. ${ }^{50,51}$ In such a case, the Twiss condition is given by $1+\alpha^{2}(\delta)=\beta(\delta) \gamma(\delta)+\sigma\left(\delta^{n+1}\right)$.

\subsection{Coupled Dispersed Betatron Motion}

Generally, even neglecting nonlinear multipole errors, the two degrees of freedom of the horizontal and vertical motiou in a storage ring are dependent on each other; that is, they are coupled, due to skew quadrupole errors or misalignment (mis-rotation). In such a case, the matrices in Eq. (5.4) should be considered as $4 \times 4$ coupled matrices. Canonical transformations of converting the parameterized Courant-Snyder matrix given by Eq. (5.4) into a parameterized normal form are thus more tedious. Use of a computer and of a numerical library of the differential algebra is necessary. Nontheless, the normalization procedures are the same as in the uncoupled case given in the last section.

One starts with the normalization of the 0 th-order $4 \times 4$ matrix $M_{0}$. This has been well understood thanks to Edwards and Teng. ${ }^{16}$ One can follow their formulas or use the eigenvectors of $M_{0}^{T}$ with the aid of a computer to obtain the 0th order $4 \times 4$ generation matrix $A_{0} .{ }^{11,52}$ Thus, Eq. (5.6) is replaced by

$$
R_{0}=\left(\begin{array}{cccc}
\cos \mu_{0}^{x} & \sin \mu_{0}^{x} & 0 & 0 \\
-\sin \mu_{0}^{x} & \cos \mu_{0}^{x} & 0 & 0 \\
0 & 0 & \cos \mu_{0}^{y} & \sin \mu_{0}^{y} \\
0 & 0 & -\sin \mu_{0}^{y} & \cos \mu_{0}^{y}
\end{array}\right)=A_{0}^{-1} M_{0} A_{0}
$$

where $\mu_{0}^{x}$ and $\mu_{0}^{y}$ are the non-dispersed betatron tunes, which are eigenvalues of the symplectic matrix $M_{0}^{T}$. Following the same order-by-order normalization procedures of the uncoupled case in the last section and bearing in mind that $4 \times 4$ matrices instead of $2 \times 2$ matrices are to be manipulated, the coupled $M(\delta)$ can also be normalized order-by-order up to the $n$th order of $\delta$ and given by

$$
\begin{aligned}
R_{n}(\delta) & =\left(\begin{array}{cccc}
\cos \mu^{x}(\delta) & \sin \mu^{x}(\delta) & 0 & 0 \\
-\sin \mu^{x}(\delta) & \cos \mu^{x}(\delta) & 0 & 0 \\
0 & 0 & \cos \mu^{y}(\delta) & \sin \mu^{y}(\delta) \\
0 & 0 & -\sin \mu^{y}(\delta) & \cos \mu^{y}(\delta)
\end{array}\right) \\
& =\left(I-\delta^{n} A_{n}\right) \cdots\left(I-\delta A_{1}\right) A_{0}^{-1} M(\delta) A_{0}\left(I+\delta A_{1}\right) \cdots\left(I+\delta^{n} A_{n}\right)+\sigma\left(\delta^{n+1}\right)
\end{aligned}
$$


where $\mu^{x}(\delta)$ and $\mu^{y}(\delta)$ are the dispersed betatron tunes, and $A_{0}$ and $\left(I+\delta^{i} A_{i}\right)$ for $i=1,2, \ldots, n$ are order-by-order generation $4 \times 4$ matrices. They are given by

$$
\begin{aligned}
& \mu^{x}(\delta)=\mu_{0}^{x}+\delta \mu_{1}^{x}+\delta^{2} \mu_{2}^{x}+\ldots+\delta^{n} \mu_{n}^{x}, \\
& \mu^{y}(\delta)=\mu_{0}^{y}+\delta \mu_{1}^{y}+\delta^{2} \mu_{2}^{y}+\ldots+\delta^{n} \mu_{n}^{y}
\end{aligned}
$$

where the perturbed tunes $\mu_{i}^{x}$ and $\mu_{i}^{y}$ for $i=1,2, \ldots, n$ are given by

$$
\mu_{i}^{x}=-\frac{\operatorname{Tr}\left({ }_{i} M_{i}^{x x}\right)}{2 \sin \mu_{0}^{x}}=-\frac{a_{i}^{x x}+d_{i}^{x x}}{2 \sin \mu_{0}^{x}}, \quad \mu_{i}^{y}=-\frac{\operatorname{Tr}\left({ }_{i} M_{i}^{y y}\right)}{2 \sin \mu_{0}^{y}}=-\frac{a_{i}^{y y}+d_{i}^{y y}}{2 \sin \mu_{0}^{y}} .
$$

Note that symbolically we have assumed that the $i$ th canonically transformed $4 \times 4$ matrix ${ }_{i} M_{i}$ in Eq. (5.11) is given by

$$
i M_{i}=\left(\begin{array}{ll}
i M_{i}^{x x} & i \cdot M_{i}^{x y} \\
i M_{i}^{y x} & i M_{i}^{y y}
\end{array}\right), .
$$

where the four $2 \times 2$ matrices $i M_{i}^{\kappa \lambda}$, for $\kappa=x, y$ and $\lambda=x, y$, are given by

$$
i M_{i}^{\kappa \lambda}=\left(\begin{array}{ll}
a_{i}^{\kappa \lambda} & b_{i}^{\kappa \lambda} \\
c_{i}^{\kappa \lambda} & d_{i}^{\kappa \lambda}
\end{array}\right) .
$$

The nil-potent $4 \times 4$ matrices $A_{i}$ for $i=1,2, \ldots n$ are given by

$$
A_{i}=\left(\begin{array}{cc}
A_{i}^{x x} & A_{i}^{x y} \\
A_{i}^{y x} & A_{i}^{y y}
\end{array}\right)
$$

where the four $2 \times 2$ matrices $A_{i}^{\kappa \lambda}$, for $\kappa=x, y$ and $\lambda=x, y$ are given by

$$
A_{i}^{\kappa \lambda}=-\frac{1}{\cos \mu_{0}^{\kappa}-\cos \mu_{0}^{\lambda}}\left(\begin{array}{cc}
0 & b_{i}^{\kappa \lambda} \\
c_{i}^{\kappa \lambda} & 0
\end{array}\right)
$$

for $\kappa=x, \lambda=y$ or $\kappa=y, \lambda=x$ and

$$
A_{i}^{\kappa \kappa}=\frac{1}{4 \sin \mu_{0}^{\alpha}}\left(\begin{array}{cc}
b_{i}^{\kappa \kappa}+c_{i}^{\kappa \kappa} & d_{i}^{\kappa \kappa}-a_{i}^{\kappa \kappa} \pm \sqrt{h_{i}^{\kappa \kappa}} \\
d_{i}^{\kappa \kappa}-a_{i}^{\kappa \kappa} \mp \sqrt{h_{i}^{\kappa \kappa}} & -\left(b_{i}^{\kappa \kappa}+c_{i}^{\kappa \kappa}\right)
\end{array}\right)
$$

for $\kappa=x$ or $\kappa=y$, where

$$
h_{i}^{\kappa \kappa}=\left(a_{i}^{\kappa \kappa}-d_{i}^{\kappa \kappa}\right)^{2}+\left(b_{i}^{\kappa \kappa}+c_{i}^{\kappa \kappa}\right)^{2}+\frac{16 b_{i}^{\kappa \kappa} c_{i}^{\kappa \kappa} \sin ^{2} \mu_{0}^{\kappa}}{\left(\cos \mu_{0}^{\kappa}-\cos \mu_{0}^{\lambda}\right)^{2}}
$$

for $\kappa \neq \lambda$. 
When comparing to the uncoupled case, note that the extra term $16 b_{i}^{\kappa \kappa} c_{i}^{\kappa \kappa} \sin ^{2} \mu_{0}^{\kappa} /\left(\cos \mu_{0}^{\kappa}-\cos \mu_{0}^{\lambda}\right)^{2}(\kappa \neq \lambda)$ appears in the formula for $h_{i}^{\kappa \kappa}$ due to the coupling between the two degrees of freedom.

Neglecting $\sigma\left(\delta^{n+1}\right)$ in Eq. (5.16), $M(\delta)$ can be represented by a $4 \times 4$ symplectic matrix $M_{s}(\delta)$ as given by Eq. (5.13). But bear in mind that those generation matrices and the rotational matrix are all $4 \times 4$ instead of $2 \times 2$ matrices.

In principle, Eq. (5.13) can be further converted into a Twiss form given by

$$
M_{s}(\delta)=C_{n}(\delta) T_{n}(\delta) C_{n}^{-1}(\delta)
$$

where all the couplings are absorbed into the parameterized symplectic $4 \times 4$ matrix $C_{n}(\delta)$ (concatenated from a series of symplectic parameterized matrices), and its inverse $C_{n}^{-1}(\delta) ; T_{n}(\delta)$ is a decoupled $4 \times 4$ Twiss matrix given by

$$
T_{n}(\delta)=\left(\begin{array}{cc}
T_{n}^{x}(\delta) & 0_{2 \times 2} \\
0_{2 \times 2} & T_{n}^{y}(\delta)
\end{array}\right)
$$

where $T_{n}^{\kappa}(\delta)$ for $\kappa=x$ or $\kappa=y$ are given by

$$
T_{n}^{\kappa}(\delta)=\left(\begin{array}{cc}
\cos \mu^{\kappa}(\delta)+\alpha^{\kappa}(\delta) \sin \mu^{\kappa}(\delta) & \beta^{\kappa}(\delta) \sin \mu^{\kappa}(\delta) \\
-\gamma^{\kappa}(\delta) \sin \mu^{\kappa}(\delta) & \cos \mu^{\kappa}(\delta)-\alpha^{\kappa}(\delta) \sin \mu^{\kappa}(\delta)
\end{array}\right)
$$

This work is currently in progress. ${ }^{53}$ The objective is to obtain the parameterized Twiss parameters explicitly as finite-order power series expansions of the energy deviation $\delta$ such that the Twiss condition is exactly satisfied.

\section{Parameterization of One-Turn Maps}

In Chapter 5, we discussed how the unavoidable energy spread in the particle beam causes dispersed closed orbits which are obtainable in the form of a power series expansion of the normalized energy deviation, $\delta=\Delta E / E_{0}$, as given by Eq. (5.1). A one-turn transverse Taylor map with respect to the dispersed closed orbit is also obtainable through the use of differential-algebraic map extraction programs such as Zmap. Such a one-turn Taylor map is parameterized with the energy deviation, $\delta$, and is given, in general, as a 4-dimensional VPS (Vector Power Series) of five variables, as shown in Eq. (5.2). Four of the five variables are the transverse canonical coordinates and momenta and are represented by the vector $\vec{x}$. The last variable is the parameter $\delta$ representing the normalized energy deviation, which is a smallness factor. We shall rewrite such a dispersedclosed-orbit Taylor map as follows: 


$$
\begin{aligned}
\vec{x}^{\prime} & =m(\vec{x}, \delta) \vec{x}=\vec{U}(\vec{x}, \delta) \\
& =M(\delta) \vec{x}+\sum_{i=0}^{n_{2}} \vec{U}_{2, i}(\vec{x}) \delta^{i}+\sum_{i=0}^{n_{3}} \vec{U}_{3, i}(\vec{x}) \delta^{i}+\cdots+\sum_{i=0}^{n_{\Omega}} \vec{U}_{\Omega, i}(\vec{x}) \delta^{i}
\end{aligned}
$$

where $M(\delta)$ is assumed to be truncated at an order $n$ of $\delta$, as given by Eq. (5.4), and $n \geq n_{2} \geq n_{3} \geq \cdots \geq n_{k} \geq \cdots \geq \cdots \geq n_{\Omega}$ in general. If the Taylor map is represented by the 4-dimensional VPS with an equally weighted order in the five variables, then we have $k+n_{k}=\Omega=n+1$ for $k=2,3, \cdots, \Omega$. Although the parameterized Tayler map given by Eq. (6.1) is not symplectic because of the truncation of high orders, it preserves order-by-order symplectic properties of the symplectic system.

In this chapter, we shall start with a discussion of the Lie transformations of such a parameterized Taylor map. The method has only recently been established. ${ }^{54}$ With the parameterized Lie transformations we can proceed to discuss faster ways of extracting the one-turn, 6-dimensional maps. We shall also discuss parameterized kick factorizations for symplectic trackings of particles. Some brief remarks about the parameterized norm form of perodic maps will also be given.

\subsection{Parameterized Lie Transformations}

The method proposed by Dragt and Finn for a series of homogeneous Lie transformations of a Taylor map representing a symplectic system without any parameter has been discussed in Chapter 3. With the inclusion of a parameter representing the energy deviation in the Taylor map, the Dragt-Finn factorization method can still be used with slight modifications. ${ }^{54}$ The key to such a parameterized Dragt-Finn factorization is the normalization of the associated parameterized Courant-Snyder matrix $M(\delta)$. The normalization algorithm was presented in Chapter 5 via order-by-order symplectic factorizations such that

$$
R_{n}(\delta)=A^{-1}(\delta) M(\delta) A(\delta)+\sigma\left(\delta^{n+1}\right),
$$

where the normalized parameterized rotation $R_{n}(\delta)$ is given by Eq. (5.16) and the canonical generation matrix $A(\delta)$ is the concatenation of the series of orderby-order canonical generation matrices; that is,

$$
A(\delta)=A_{0}\left(I+\delta^{1} A_{1}\right)\left(I+\delta^{2} A_{2}\right) \cdots\left(I+\delta^{n} A_{n}\right),
$$

where $A_{i}$ for $i=1,2, \cdots, n$ are all nil-potent matrices given by Eq. (5.18). Note that the inverse of the normalized parameterized rotation $R_{n}(\delta)$ is also a 
decoupled rotation given by

$$
R_{n}^{-1}(\delta)=\left(\begin{array}{cccc}
\cos \mu^{x}(\delta) & -\sin \mu^{x}(\delta) & 0 & 0 \\
\sin \mu^{x}(\delta) & \cos \mu^{x}(\delta) & 0 & 0 \\
0 & 0 & \cos \mu^{y}(\delta) & -\sin \mu^{y}(\delta) \\
0 & 0 & \sin \mu^{y}(\delta) & \cos \mu^{y}(\delta)
\end{array}\right),
$$

where $\mu^{x}(\delta)$ and $\mu^{y}(\delta)$ are given by Eq. (5.17). The inverse of the canonical generation matrix $A(\delta)$ is given by

$$
A^{-1}(\delta)=\left(I-\delta^{n} A_{n}\right) \cdots\left(I-\delta^{2} A_{2}\right)\left(I-\delta A_{1}\right) A_{0}^{-1} .
$$

\subsubsection{Lie-Transformation Procedures}

To convert the parameterized Taylor map given by Eq. (6.1) to a series of homogeneous Lie transformations, one first makes a similarity transformation on the map such that

$$
\begin{aligned}
{ }_{1} m(\vec{x}, \delta) \vec{x} & =\mathcal{A}(\vec{x}, \delta) m(\vec{x}, \delta) \mathcal{A}^{-1}(\vec{x}, \delta) \vec{x} \\
& =R_{n}(\delta) \vec{x}+\sum_{i=0}^{n_{2}}{ }_{1} \vec{U}_{2, i}(\vec{x}) \delta^{i}+\sum_{i=0}^{n_{3}}{ }_{1} \vec{U}_{3, i}(\vec{x}) \delta^{i}+\cdots+\sum_{i=0}^{n_{\Omega}}{ }_{1} \vec{U}_{\Omega, i}(\vec{x}),
\end{aligned}
$$

where $\mathcal{A}(\vec{x}, \delta)$ is the global form of $A(\delta)$. One then make a concatenation of the transformed map ${ }_{1} m(\vec{x}, \delta)$ with $\mathcal{R}_{n}^{-1}(\vec{x}, \delta)$ such that

$$
\begin{aligned}
{ }_{2} m(\vec{x}, \delta) \vec{x} & =\mathcal{R}_{n}^{-1}(\vec{x}, \delta)_{1} m(\vec{x}, \delta) \vec{x} \\
& =\vec{x}+\sum_{i=0}^{n_{2}}{ }_{2} \vec{U}_{2, i}(\vec{x}) \delta^{i}+\sum_{i=0}^{n_{3}}{ }_{2} \vec{U}_{3, i}(\vec{x}) \delta^{i} \cdots+\sum_{i=0}^{n_{\Omega}}{ }_{2} \vec{U}_{\Omega, i}(\vec{x}),
\end{aligned}
$$

where $\mathcal{R}_{n}^{-1}(\vec{x}, \delta)$ is the global form of the inverse of the rotational matrix $R_{n}(\delta)$. Note that the global form of the rotational matrix $R_{n}(\delta)$ is given by

$$
\mathcal{R}_{n}(\vec{x}, \delta)=e^{: f_{2}(\vec{x}, \delta):}
$$

while its inverse is given by

$$
\mathcal{R}_{n}^{-1}(\vec{x}, \delta)=e^{:-f_{2}(\vec{x}, \delta)}
$$

where

$$
f_{2}(\vec{x}, \delta)=-\frac{\mu^{x}(\delta)}{2}\left(x^{2}+p_{x}^{2}\right)-\frac{\mu^{y}(\delta)}{2}\left(y^{2}+p_{y}^{2}\right)
$$

Due to symplecticity of the Taylor map (when it is expanded to the infinite order), for each of the ${ }_{2} \vec{U}_{2, i}(\vec{x})$ for $i=0,1, \cdots, n_{2}$ there exists a homogeneous 
third-order polynomial of $\vec{x}, f_{3, i}(\vec{x})$, such that

$$
\left[f_{3, i}(\vec{x}), \vec{x}\right]={ }_{2} \vec{U}_{2, i}(\vec{x}),
$$

where [,] is the Poisson bracket. Therefore, from Eq. (6.2), one obtains

$$
{ }_{2} m(\vec{x}, \delta) \vec{x}=\exp \left(: \sum_{i=0}^{n_{2}} f_{3, i}(\vec{x}) \delta^{i}:\right) \vec{x}+\sum_{i=0}^{n_{3}}{ }_{2} \vec{U}^{\prime}{ }_{3, i}(\vec{x}) \delta^{i}+\cdots+\sum_{i=0}^{n_{\Omega}}{ }_{2} \vec{U}_{\Omega, i}(\vec{x}) \delta^{i},
$$

where

$$
{ }_{2} \vec{U}_{k, i}^{\prime}(\vec{x})={ }_{2} \vec{U}_{k, i}(\vec{x})-: f_{3, i}(\vec{x}):{ }^{k-1} \vec{x},
$$

for $k=3,4, \cdots, \Omega$. For example,

$$
{ }_{2} \vec{U}_{3, i}(\vec{x})={ }_{2} \vec{U}_{3, i}(\vec{x})-: f_{3, i}(\vec{x}):^{2} \vec{x}={ }_{2} \vec{U}_{3, i}(\vec{x})-\left[f_{3, i}(\vec{x}),\left[f_{3, i}(\vec{x}), \vec{x}\right]\right] .
$$

Concatenating $2 m(\vec{x}, \delta)$ with

$$
\exp \left(-: \sum_{i=0}^{n_{2}} f_{3, i}(\vec{x}) \delta^{i}:\right)
$$

one obtains

$$
\begin{aligned}
{ }_{3} m(\vec{x}, \delta) \vec{x} & =\exp \left(-: \sum_{i=0}^{n_{2}} f_{3, i}(\vec{x}) \delta^{i}:\right)_{2} m(\vec{x}, \delta) \vec{x} \\
& =\vec{x}+\sum_{i=0}^{n_{3}}{ }_{3} \vec{U}_{3, i}(\vec{x}) \delta^{i}+\sum_{i=0}^{n_{4}}{ }_{3} \vec{U}_{4, i}(\vec{x}) \delta^{i}+\cdots+\sum_{i=0}^{n_{\Omega}}{ }_{3} \vec{U}_{\Omega, i}(\vec{x}) \delta^{i}
\end{aligned}
$$

Again, due to symplecticity, for each of the ${ }_{3} \vec{U}_{3, i}(\vec{x})$ for $i=0,1, \cdots, n_{3}$, there exists a homogeneous fourth-order polynomial of $\vec{x}, f_{4, i}(\vec{x})$, such that

$$
\left[f_{4, i}(\vec{x}), \vec{x}\right]={ }_{3} \vec{U}_{3, i}(\vec{x}) \text {. }
$$

One thus obtains the Dragt-Finn factorization of the fourth order given by

$$
\exp \left(: \sum_{i=0}^{n_{3}} f_{4, i}(\vec{x}) \delta^{i}:\right)
$$

Following steps similar to those given above, one can proceed further to obtain the Dragt-Finn factorization of the higher orders given by

$$
\exp \left(: \sum_{i=0}^{n_{k}} f_{k+1, i}(\vec{x}) \delta^{i}:\right)
$$

for $h=4,5, \cdots, \Omega$. Truncating the high-order terms beyond our interest, the parameterized Taylor map given by Eq. (6.1) can then be representzd, in a 
global form, by

$$
\overrightarrow{x^{\prime}}=m(\vec{x}, \delta) \vec{x}=\mathcal{A}^{-1}(\vec{x}, \delta) m_{f} \mathcal{A}(\vec{x}, \delta) \vec{x},
$$

where

$$
m_{f}=\mathcal{R}_{n}(\vec{x}, \delta) \exp \left(: f_{3}(\vec{x}, \delta):\right) \ldots \exp \left(: f_{k}(\vec{x}, \delta):\right) \ldots \exp \left(: f_{\Omega+1}(\vec{x}, \delta):\right),
$$

where for each $k=1,2, \ldots, \Omega+1$,

$$
f_{k}(\vec{x}, \delta)=\sum_{i=0}^{n_{k-1}} f_{k, i}(\vec{x}) \delta^{i}
$$

Recalling that $\mathcal{R}_{n}=\exp \left(: f_{2}(\vec{x}, \delta):\right)$, where $f_{2}(\vec{x}, \delta)$ is given by Eq. (6.3), the parameterized Taylor map can also be represented completely by a series of parameterized Lie transformations given in global form by

$$
\begin{aligned}
\overrightarrow{x^{\prime}} & =m(\vec{x}, \delta) \vec{x}=\mathcal{A}^{-1}(\vec{x}, \delta) e^{: f_{2}(\vec{x}, \delta):} e^{: f_{3}(\vec{x}, \delta): \ldots e^{: f_{\Omega+1}(\vec{x}, \delta):} \mathcal{A}(\vec{x}, \delta) \vec{x}} \\
& =\mathcal{A}^{-1}(\vec{x}, \delta) e^{: f_{2}(\vec{x}, \delta):} \mathcal{A}(\vec{x}, \delta) \mathcal{A}^{-1}(\vec{x}, \delta) \cdots \mathcal{A}(\vec{x}, \delta) \mathcal{A}^{-1}(\vec{x}, \delta) e^{: f_{\Omega+1}(\vec{x}, \delta):} \mathcal{A}(\vec{x}, \delta) \vec{x} \\
& =e^{: f_{2}^{\prime}(\vec{x}, \delta):} e^{: f_{3}^{\prime}(\vec{x}, \delta):} \ldots e^{: f_{\Omega+1}^{\prime}(\vec{x}, \delta):} \vec{x}
\end{aligned}
$$

where for $k=2,3, \cdots, \Omega+1$,

$$
e^{: f_{k}^{\prime}(\vec{x}, \delta):}=\mathcal{A}^{-1}(\vec{x}, \delta) e^{: f_{k}(\vec{x}, \delta):} \mathcal{A}(\vec{x}, \delta)=e^{: \mathcal{A}^{-1}(\vec{x}, \delta) f_{k}(\vec{x}, \delta):}=e^{: f_{k}\left(A^{-1}(\delta) \vec{x}, \delta\right):} .
$$

\subsubsection{Further Justifications}

At first glance, one may wonder why Eq. (6.4) [or Eq. (6.5)] is true. Considering that it is one of the important steps leading to the parameterized Dragt-Finn factorization of a dispersed-closed-orbit Taylor map, further justification is given in this section.

Let us assume that the Taylor expansions are all infinite orders of $\delta$ that is, $n=n_{2}=n_{3}=\cdots=\infty$. If a suitable small factor is substituted for $\delta$ into Eq. (6.1), then one obtains the associated regular closed-orbit, nonparameterized Taylor map, which is a familiar type of map for Dragt-Finn factorization. Now if we first obtain the parameterized normalized rotation $R(\delta)$ and its associated parameterized canonical generation matrix $A(\delta)$ up to the infinite order of $\delta$, and then substitute the same small factor for $\delta$, we obtain the same linear, non-parameterized, normalized rotation $R$ and its associated non-parameterized canonical generation matrix $A$ as those obtained for the associated non-parameterized Dragt-Finn factorization. Therefore, it makes no 
difference whether we first obtain Eq. (6.2) to infinite order of $\delta$ and then substitute the small factor for $\delta$, or we first substitute the small factor for $\delta$ in Eq. (6.1) to obtain a non-parameterized, closed-orbit Taylor map and then obtain the associated non-parameterized equivalence of Eq. (6.2). Thus, because of symplecticity, there must exist a homogeneous third-order polynomial of $\vec{x}$, $f(\vec{x}, \delta)$, such that

$$
[f(\vec{x}, \delta), \vec{x}]=\sum_{i=0}^{\infty} \vec{U}_{2, i}(\vec{x}) \delta^{i}
$$

Since $f(\vec{x}, \delta)$ can always be Taylor-expanded as

$$
f(\vec{x}, \delta)=\sum_{i=0}^{\infty} f_{i}(\vec{x}) \delta^{i},
$$

by comparing Eq. (6.9) and Eq. (6.10), one concludes that

$$
\left[f_{i}(\vec{x}), \vec{x}\right]={ }_{2} \vec{U}_{2, i}(\vec{x})
$$

for each $i=0,1,2, \cdots, \infty$. Furthermore, since terms with a certain order of $\delta$ can be contributed only through concatenation from terms with lower or equal orders of $\delta$, truncation of higher-order terms will not change the outcome for the lower-order Lie operators. Therefore, $f_{3, i}(\vec{x})=f_{i}(\vec{x})$ for $i=0,1, \cdots, n_{2}$.

\subsection{Faster Extraction of One-Turn 6-D Maps}

Generally, for long-term stability studies of the storage rings (as discussed in Chapter 4), one would prefer 6-dimensional maps to 4-dimensional transverse maps so that synchrotron oscillations can be included. The most straightforward way of obtaining such a 6-dimensional map is to extract the one-turn Taylor map with six variables. The six variables are

$$
\vec{z}^{T}=\left(\vec{x}^{T}, \tau, p_{\tau}\right)=\left(x, p_{x}, y, p_{y}, \tau,-\delta\right),
$$

where the extra variable $\tau$ is the normalized time of flight, as discussed in Chapter 2 . In this case, the normalized energy deviation $\delta$ is no longer a parameter; $-\delta$ is the conjugate momentum of the time of flight $\tau$. For convenience, in this section we shall consider $\delta$ as the longitudinal conjugate coordinate and $\tau$ as the longitudinal conjugate momentum (with a simple canonical transformation). Thus the vector $\vec{z}$ representing the 6 phase-space coordinates is given by

$$
\vec{z}^{T}=\left(\vec{x}^{T}, \delta, \tau\right)=\left(x, p_{x}, y, p_{y}, \delta, \tau\right) .
$$

For cases with a single RF cavity which is modeled as a thin RF kick and drifts (as discussed in Chapter 5), there are faster ways of obtaining the one-turn Taylor maps. 


\subsubsection{Conversion from 4-by-5 and 1-by-5 maps}

One common method of more quickly obtaining the 6-dimensional map is presented here. First, between one RF kick and the next RF kick, one obtains a 4-dimensional transverse map, represented by a 4-dimensional VPS of five variables $\left(x, p_{x}, y, p_{y}, \delta\right)$, and a 1-dimensional map for the advancement of time of flight, $\Delta \tau$, represented by a PS (a 1-dimensional VPS) of the same five variables $\left(x, p_{x}, y, p_{y}, \delta\right)$; that is, one obtains

$$
\vec{x}^{\prime}=\vec{U}(\vec{x}, \delta)
$$

and

$$
\Delta \tau=V(\vec{x}, \delta),
$$

where $\delta$ is temporarily treated as a parameter since it can be changed only with RF kicks. At this stage, the 4-by-5 map and 1-by-5 map, given by Eq. (6.11) and Eq. (6.12), respectively, together with the separated thin RF kick, can be used for particle trackings. A complete one-turn track would include two steps: (1) particles are tracked over the 4-by-5 map given by Eq. (6.11) to update their transverse coordinates $(x, y)$ and momenta $\left(p_{x}, p_{y}\right)$ and over the 1-by-5 map given by Eq. (6.12) to obtain the advancement of the time of flight $\Delta \tau$; and (2) the time of flight $\tau$ is then updated by adding $\Delta \tau$ to the initial time of flight, and so the energy deviation is updated with the thin RF kick. This is how those long-term Taylor map trackings, with which the survival plots are shown in Chapter 4, were performed.

To complete the extraction of the 6-dimensional, one-turn Taylor map of six variables (6-by- 6 map), one then converts the 4-by-5 map given by Eq. (6.11) and the 1-by- 5 map given by Eq. (6.12) into a 4 -by- 6 map and a 1-by- 6 map given, respectively, as

and

$$
\begin{gathered}
\vec{x}=\vec{U}(\vec{x}, \delta, \tau) \\
\Delta \tau=V(\vec{x}, \delta, \tau) .
\end{gathered}
$$

Note that all the coefficients are 0 for those monomials with non-zero orders in $\tau$. The 1-by- 6 map for $\tau$ is obtained by performing PS addition of $\tau$ and $V(\vec{x}, \delta, \tau)$ given by Eq. (6.14); that is,

$$
\tau^{\prime}=U^{\prime}(\vec{x}, \delta, \tau)=V(\vec{x}, \delta, \tau)+\tau .
$$

With concatenation over the thin RF kick, one obtains the 1-by-6 map for the energy deviation $\delta$, which (let us assume) is given by

$$
\delta^{\prime}=U^{\prime \prime}(\vec{x}, \delta, \tau) \text {. }
$$

Combination of the 4-by-6 map given by Eq. (6.13) and the two 1-by-6 maps given by Eq. (6.15) and Eq. (6.16) yields the one-turn, 6-by-6 map. 


\subsubsection{Conversion from 4-by-5 and 1-by-1 maps}

Another, even faster, way to obtain the 6-dimensional map is as follows. First, one obtains the dispersed-closed-orbit 4-by-5 Taylor map as given by Eq. (6.1), and then converts it into a series of homogeneous (in $\vec{x}$ ) Lie transformations given by Eq. (6.6) or Eq. (6.8). Next, the dispersed-closed-orbit map (Lie transformations) is shifted to a regular closed-orbit map [with respect to $\vec{x}_{c, 0}$ instead of $\vec{x}_{c}(\delta)$ given by Eq. (5.1)], given by

$$
\vec{x}^{\prime}=e^{:-f_{1}^{\prime}(\vec{x}, \delta):} e^{: f_{2}^{\prime}(\vec{x}, \delta):} e^{: f_{3}^{\prime}(\vec{x}, \delta):} \cdots e^{: f_{\Omega+1}^{\prime}(\vec{x}, \delta):} e^{: f_{1}^{\prime}(\vec{x}, \delta):} \vec{x},
$$

where

$$
f_{1}^{\prime}(\vec{x}, \delta)=\vec{x}^{T} S \vec{x}_{c}^{\prime}(\delta)
$$

and

$$
\vec{x}_{c}^{\prime}(\delta)=\vec{x}_{c}(\delta)-\vec{x}_{c, 0} .
$$

To obtain the 1-by-5 or 1-by-6 Taylor map for $\tau$, it is natural to consider use of the Lie transformation in Eq. (6.17), since $\tau$ is the canonical conjugate momentum of $\delta$. However, the information for the monomials of the Taylor map for $\tau$, with 0th order in $\vec{x}$, is not contained in the original 4-by-5 Taylor map and so cannot be obtained from the Lie transformation. Fortunately, on the one $\mathrm{f}_{1}$ and the 4-by-5 map for $\vec{x}$ is still the same if we add any Lie transformation given by $\exp \left(: f_{0}(\delta):\right)$ to Eq. (6.17), and on the other hand, a PS $f_{0}(\delta)$ is obtainable that would carry the correct information for the map of $\tau$. To obtain the PS $f_{0}(\delta)$ for the map of $\tau$, simply extract, with the map extraction program, a 1-by-1 Taylor map $V_{0}(\delta)$ for $\tau$ that gives the monomials with 0th order in $\vec{x}$, then let

$$
f_{0}(\delta)=\int V_{0}(\delta) d \delta
$$

Adding $\exp \left(: f_{0}(\delta):\right)$ to Eq. (6.17), one obtains

$$
\vec{x}=e^{: f_{0}(\delta):} e^{:-f_{1}^{\prime}(\vec{x}, \delta):} e^{: f_{2}^{\prime}(\vec{x}, \delta):} e^{: f_{3}^{\prime}(\vec{x}, \delta): \ldots} e^{: f_{\Omega+1}^{\prime}(\vec{x}, \delta):} e^{: f_{1}^{\prime}(\vec{x}, \delta):} \vec{x}
$$

and

$$
\tau^{\prime}=e^{: f_{0}(\delta):} e^{:-f_{1}^{\prime}(\vec{x}, \delta):} e^{: f_{2}^{\prime}(\vec{x}, \delta):} e^{: f_{3}^{\prime}(\vec{x}, \delta):} \cdots e^{: f_{n+1}^{\prime}(\vec{x}, \delta):} e^{: f^{\prime}(\vec{x}, \delta):} \tau .
$$

Since the Lie transformations given by Eq. (6.19) or Eq. (6.20) constitute an identity map for $\delta$-that is,

$$
\delta^{\prime}=e^{: f_{0}(\delta):} e^{:-f_{1}^{\prime}(\vec{x}, \delta):} e^{: f_{2}^{\prime}(\vec{x}, \delta):} e^{: f_{3}^{\prime}(\vec{x}, \delta):} \cdots e^{: f_{\Omega+1}^{\prime}(\vec{x}, \delta)}: e^{: f_{1}^{\prime}(\vec{x}, \delta):} \delta=\delta,
$$


the combination of Eqs. (6.19), (6.20), and (6.21) gives the 6-by-6 Lie map from after the RF kick to before the RF kick:

$$
\vec{z}^{\prime}=e^{: f_{0}(\delta):} e^{:-f_{1}^{\prime}(\vec{x}, \delta):} e^{: f_{2}^{\prime}(\vec{x}, \delta):} e^{: f_{3}^{\prime}(\vec{x}, \delta):} \ldots e^{: f_{\Omega+1}^{\prime}(\vec{x}, \delta):} e^{: f_{1}^{\prime}(\vec{x}, \delta):} \vec{z},
$$

where

$$
\vec{z}^{T}=\left(\vec{x}^{T}, \delta, \tau\right)=\left(x, p_{x}, y, p_{y}, \delta, \tau\right) .
$$

The Lie transformation of the RF kick can be easily formulated such that

$$
\delta^{\prime}=e^{: g(\tau): \delta}
$$

or

$$
\vec{z}^{\prime}=e^{: g(\tau):} \vec{z}
$$

since the Lie transformation $e^{: g(\tau):}$ is an identity map for $\vec{x}$ and $\tau$. The final 6-by-6, one-turn map is given by

$$
\vec{z}^{\prime}=e^{: f_{0}(\delta):} e^{:-f_{1}^{\prime}(\vec{x}, \delta):} e^{: f_{2}^{\prime}(\vec{x}, \delta):} e^{: f^{\prime}(\vec{x}, \delta):} \cdots e^{: f_{\Omega+1}^{\prime}(\vec{x}, \delta):} e^{: f_{1}^{\prime}(\vec{x}, \delta):} e^{: g(\tau):} \vec{z} .
$$

If necessary, expansion of Eq. (6.24) would give the 6-by-6 Taylor map.

Note that this conversion method is about twice as fast as the one discussed in the last section for the SSC lattice. This is because extraction of a 4-by5 Taylor map for $\vec{x}$ accompanied by a 1-by- 5 Taylor map for $\tau$ takes about twice as much time as simple extraction of a 4-by-5 Taylor map for $\vec{x}$. Computer time used for the extraction of the 1-by-1 Taylor map $V_{0}(\delta)$ and for the conversion process is negligible compared with the computer time needed for extracting the 4-by-5 Taylor map for the SSC.

\subsection{Parameterized Kick Factorizations}

In Chapter 3, we discussed how a non-parameterized Dragt-Finn factorization map, $m_{f}$, given by Eq. (3.14), was converted into kicks, given by Eq. (3.15), such that the map $m_{f}$ can be symplectically evaluated with an accuracy up to order $\Omega$ in terms of the Taylor expansion. Then, in Chapter 4, we briefly commented that a 6-dimensional kick-map tracking is generally slower than the corresponding re-expanded, suitably high-order Taylor map tracking. To make the speed of kick-map tracking more competitive, we shall, in this section, discuss how the kick factorizations are parameterized such that one can consider 4-dimensional instead of 6-dimensional kick-map factorizations, thereby enhancing the tracking speed. 
Consider the same Irwin kick factorization basis set $\vec{\xi}=\left\{\vec{\xi}_{1}, \vec{\xi}_{2}, \ldots \vec{\xi}_{k}, \ldots\right.$ $\left.\vec{\xi}_{\Gamma}\right\}$ discussed in Section 3.7.1. Then the parameterized Dragt-Finn factorization map $m_{f}$ given by Eq. (6.7) can be converted into parameterized kick factorizations given by

$$
m_{g}=\mathcal{R}_{n}(\vec{x}, \delta) \prod_{k=1}^{\Gamma} e^{: g_{k}\left(\vec{\xi}_{k}, \delta\right):}=\mathcal{R}_{n}(\vec{x}, \delta) \prod_{k=1}^{\Gamma} \mathcal{R}_{k}(\vec{x}) e^{: g_{k}(\vec{\xi}, \delta):} \mathcal{R}_{k}^{-1}(\vec{x}),
$$

where

$$
\begin{aligned}
g_{k}\left(\vec{\xi}_{k}, \delta\right) & =\sum_{i=0}^{n} g_{k, i}\left(\vec{\xi}_{k}\right) \delta^{i}=\sum_{i=0}^{n} g_{k, i}\left(\vec{x}_{k}\right) \delta^{i}=\sum_{i=0}^{n} g_{k, i}\left(R_{k} \vec{x}\right) \delta^{i} \\
& =\sum_{i=0}^{n} \mathcal{R}_{k}(\vec{x}) g_{k, i}(\vec{x}) \delta^{i}=\mathcal{R}_{k}(\vec{x}) \sum_{i=0}^{n} g_{k, i}(\vec{\xi}) \delta^{i}=\mathcal{R}_{k}(\vec{x}) g_{k}(\vec{\xi}, \delta)
\end{aligned}
$$

Note that $\vec{x}^{T}=\left(x, p_{x}, y, p_{y}\right), \vec{\xi}^{T}=(x, y), \vec{x}_{k}^{T}=\left(x_{k}, p_{x k}, y_{k}, p_{y k}\right), \vec{\xi}_{k}^{T}=\left(x_{k}, y_{k}\right)$; $n$ is the maximum of $n_{2}, n_{3}, \ldots, n_{\Omega}$. Also note that we have let $g_{k, i}\left(\vec{\xi}_{k}\right)=$ $g_{k, i}\left(\vec{x}_{k}\right)$ and $g_{k, i}(\vec{x})=g_{k, i}(\vec{\xi})$ because the coefficients for the monomials with non-zero order in conjugate momenta- $-\left(p_{x k}, p_{y k}\right)$ or $\left(p_{x}, p_{y}\right)$-are all 0 for the power series $g_{k, i}(\vec{x})$.

To obtain Eq. (6.25), factorization procedures discussed in Section 3.7 are followed with slight modification to treat the parameter $\delta$. For example, let us consider converting a parameterized homogeneous Lie transformation of degree $i$ (in $\vec{x}$ ) given by

$$
\exp \left(: f_{i}(\vec{x}, \delta):\right)=\exp \left(: \sum_{j=0}^{n} f_{i j}(\vec{x}) \delta^{j}:\right)
$$

into parameterized kick factorizations. We follow exactly the same procedure as in Section 3.7.2 to decompose each of $f_{i j}(\vec{x})$ for $j=0, \ldots, n$ such that

$$
f_{i j}(\vec{x})=\sum_{k=1}^{\Gamma} g_{i j k}\left(x_{k}, y_{k}\right) \text {. }
$$

Then

$$
f_{i}(\vec{x}, \delta)=\sum_{j=0}^{n}\left(\sum_{k=1}^{\Gamma} g_{i j k}\left(x_{k}, y_{k}\right)\right) \delta^{i}=\sum_{k=1}^{\Gamma} \sum_{j=0}^{n} g_{i j k}\left(x_{k}, y_{k}\right) \delta^{i}=\sum_{k=1}^{\Gamma} g_{i k}\left(x_{k}, y_{k}, \delta\right) .
$$


It can be proved that

$$
e^{: f_{i}(\vec{x}, \delta):} \vec{x}=\prod_{k=1}^{\Gamma} e^{: g_{i k}\left(x_{k}, y_{k}, \delta\right):} \vec{x}+\sigma(i) .
$$

Then, following the order-by-order kick factorization procedure in Section 3.7.3, we would obtain Eq. (6.25).

Each $\exp \left(: g_{k}(\vec{\xi}, \delta):\right)$ in Eq. (6.25) for $k=1,2, \ldots, \Gamma$ is a kick given by

$$
\left(\begin{array}{c}
p_{x}^{\prime} \\
p_{y}^{\prime}
\end{array}\right)=e^{: g_{k}(x, y, \delta):}\left(\begin{array}{c}
p_{x} \\
p_{y}
\end{array}\right)=\left(\begin{array}{c}
p_{x}+\Delta p_{x k}(x, y, \delta) \\
p_{y}+\Delta p_{y k}(x, y, \delta)
\end{array}\right)
$$

where $\Delta p_{x k}(x, y, \delta)=\partial g_{k}(x ; y, \delta) / \partial x$ and $\Delta p_{y k}(x, y, \delta)=\partial g_{k}(x, y, \delta) / \partial y$. Note that at each given turn, $\delta$ is a constant so that each of the 3-variable polynomials $\Delta p_{x k}$ and $\Delta p_{y k}$ can be reduced to 2-variable polynomials through a single substitution of the value of $\delta$ for all of the $\Gamma$ kicks.

With respect to the regular closed orbit (not the dispersed closed orbit), the kick map for the transverse phase-space coordinates is given by

$\vec{x}^{\prime}=e^{:-f_{1}^{\prime}(\vec{x}, \delta):} \mathcal{A}^{-1}(\vec{x}, \delta) \mathcal{R}_{n}(\vec{x}, \delta)\left(\prod_{k=1}^{\Gamma} \mathcal{R}_{k}(\vec{x}) e^{: g_{k}(\vec{\xi}, \delta):} \mathcal{R}_{k}^{-1}(\vec{x})\right) \mathcal{A}(\vec{x}, \delta) e^{: f_{1}^{\prime}(\vec{x}, \delta)} \vec{x}$,

where $f_{1}^{\prime}(\vec{x}, \delta)$ is given by Eq. (6.18). As for updating the one-turn time of flight, $\tau$, Eq. (6.20) can still be used since it is already a kick format for $\tau$. To update the energy deviation $\delta$ through RF kick, we use Eq. (6.23). The energy deviation is updated as follows:

$$
\delta^{\prime}=\delta+\left.\frac{\partial g(\tau)}{\partial \tau}\right|_{\tau^{\prime}}
$$

\subsection{Parameterized Normal Forms}

Bearing in mind that the betatron tunes $\mu^{x}(\delta)$ and $\mu^{y}(\delta)$ given by Eq. (5.17) are parameterized, and following the procedures discussed in Section 3.8 with slight modification, one can obtain the parameterized normal form for the parameterized map $m_{f}$ given by Eq. (6.7):

$$
m_{f}=G^{-1}(\vec{x}, \delta) e^{: h(\vec{J}, \delta):} G(\vec{x}, \delta)+\sigma(\Omega+1),
$$


where the canonical generator $G(\vec{x}, \delta)$ is the concatenation of a series of canonical Lie generators given by

$$
\begin{gathered}
G(\vec{x}, \delta)=\prod_{i=3}^{\Omega+1} e^{: F_{i}(\vec{x}, \delta):} \\
\vec{J}^{T}=\left(J_{x}, J_{y}\right), \quad J_{x}=\frac{1}{2}\left(x^{2}+p_{x}^{2}\right), \quad J_{y}=\frac{1}{2}\left(y^{2}+p_{y}^{2}\right),
\end{gathered}
$$

and

$$
e^{: h(\vec{J}, \delta):}=e^{-\vec{\mu}(\delta) \cdot \vec{J}} \exp \left(: \sum_{i=3}^{\Omega+1} h_{i}(\vec{J}, \delta)\right)=\mathcal{R}(\vec{x}, \delta) \exp \left(: \sum_{i=3}^{\Omega+1} h_{i}(\vec{J}, \delta)\right) .
$$




\section{Acknowledgements}

The author would like to thank Prof. Alex Dragt and Dr. Martin Berz, Dr. Etienne Forest, and Dr. John Irwin for many illuminating discussions. He would also like to thank Dr. Jack Peterson and Dr. Richard Talman for many useful suggestions and Dr. Tanaji Sen for his collaboration over the last one-anda-half years. It was during this time that the long-term Taylor map trackings were performed for the SSC injection lattice and, to the surprise of most of our colleagues, the differential algebraic one-turn map of moderate order has worked very well. The encouragement and patience of Dr. Melvin Month are highly appreciated.

This paper could not have been finished without the encouragement and support of Dr. Alex Chao and Dr. Mike Syphers. 


\section{References}

1. E. Courant and H. Snyder, "Theory of the Alternating-Gradient Synchrotron," Ann. Phys., 3, 1 (1958).

2. J.R. Sanford and D.M. Matthews, eds., "Site-Specific Conceptual Design of the Superconducting Super Collider," Superconducting Super Collider Laboratory Report SSCL-SR-1056 (1990).

3. M. Berz, "Differential Algebraic Description of Beam Dynamics to Very High Orders," Particle Accel. 24, 109 (1989).

4. A. Dragt and J. Finn, "Lie Series and Invariant Functions for Analytic Symplectic Maps," J. Math. Phys., 17, 2215 (1976).

5. A. Robinson, Proc. Royal Acad. Amsterdam Ser. A, 64, 432 (1961).

6. D. Laugwitz, JBer. Deutsh. Math. Vereinigung, 75, 66 (1973).

7. L.B. Rall, Math. Magazine, 59, 275 (1986).

8. I. Niven, Am. Math. Monthly, 76-8, 871 (1969).

9. M. Berz, "DA-package: a package of computer programs for performing differential algebra." It uses Fortran language and is scalarly optimized.

10. L. Michelotti, "MXYZPPLK: a C++ version of differential algebra," Fermi National Accelerator Laboratory Report FN-535 (1990).

11. Y. Yan and C. Yan, "Zlib-A Numerical Library for Differential Algebra," SSC Laboratory Report SSCL-300 (1990). The source of this numerical library uses Fortran language. Dynamic memories are employed. It is vectorized and parallelized (multi-tasking) for supercomputers.

12. A. Dragt et al., "Lie Algebraic Treatment of Linear and Nonlinear Beam Dynamics," Ann. Rev. Nucl. Part. Sci., 38, 455 (1988).

13. J.E. Campbell, Proc. London Math. Soc., 29, 14 (1898).

14. H.F. Baker, Proc. London Math. Soc., 34, 347 (1902).

15. F. Hausdorf, Ber. Verhandl. Akad. Wiss. Leipzig, Math-naturwiss, 58, 19 (1906).

16. D. Edwards and L. Teng, "Parameterization of the Linear Coupled Motion in Periodic Systems," in Proc. 1979 IEEE Particle Accelerator Conf., p. 885 (1973); L.C. Teng, Fermi National Accelerator Laboratory Report FN-229 (1971).

17. J. Irwin, "A Multi-Kick Factorization Algorithm for Nonlinear Maps," SSC Report SSC-228 (1989).

18. A. Dragt, "Method for Symplectic Tracking," presented at Workshop on Nonlinear Problems in Future Particle Accelerators, Capri, Italy (April 1990).

19. A. Deprit, Celestial Mechanics, 1, 12 (1969).

20. G. Hori, Publications of the Astronomical Society of Japan, 18, 287 (1966).

21. A.J. Dragt and J.M. Finn, "Normal Form for Mirror Machine Hamiltonians," Journal of Mathematical Physics, 20(12), 2649 (1979).

22. J.R. Cary, Phys. Rep., 79, 129-159 (1981). 
23. L. Michelotti, Particle Accelerators, 16, 233 (1985).

24. E. Forest, "Normal Form Algorithm on Non-Linear Symplectic Maps," SSC Laboratory Report SSC-29 (1985).

25. A. Bazzani, P. Mazzanti, G. Servizi, and G. Turchetti, "Normal Forms for Hamiltonian Maps and Nonlinear Effects in a Particle Accelerator," Il Nuovo Cimento, 102 B, N.1, 51 (1988).

26. A. Bazzani, B. Leeman, W. Scandale, G. Servizi, and G. Turchetti, "Description of Nonlinear Beam Dynamics in the CERN Large Hadron Collider by Using Normal Form Algorithms," Proc. of European Part. Accel. Conf., 2, 887, Rome (June, 1988).

27. E. Forest, M. Berz, and J. Irwin, "Normal Form Methods for Complicated Periodic Systems," Particle Accelerators, 24, 91 (1989).

28. E. Keil, "Computer Programs in Accelerator Physics," AIP Conf. Proc., 153, 83 (1985), M. Month and M. Dienes, eds.

29. L. Schachinger and R. Talman, "Teapot: A Thin-Element Accelerator Program for Optics and Tracking," Particle Accelerators, 22, 35 (1987).

30. Y. Yan, G. Bourianoff, and L. Schachinger, "A Typical 'Ztrack' LongTerm Tracking Result," SSC Laboratory Report SSCL-303 (1990); Proc. Workshop on Nonlinear Problems in Future Particle Accelerators, Capri, Italy (April 1990).

31. Y. Yan, "Zmap-A Differential Algebraic High-Order Map Extraction Program for Teapot Using ZLIB," SSC Laboratory Report SSCL-299 (1990).

32. L. Schachinger and Y. Yan, "Recent SSC Dynamic Aperture Measurements from Simulations," SSC Laboratory Note SSC-N-664 (1989).

33. Y. Yan, "Supercomputing for the Superconducting Super Collider," Energy Sciences Supercomputing 1990, pp. 9-13 (1990), DOE National Energy Research Supercomputer Center, A. Mirin and G. Kaiper, eds.

34. T. Garavaglia, S.K. Kauffmann, R. Stiening, and D. Ritson, "Numerical Studies of the SSC Injection Process: Long-Term Tracking," SSC Laboratory Report SSCL-268 (1990).

35. A.A. Garren, "Lattice of the SSC," Proc. Intl. High Energy Accel. Ccnf., Novosibersk (1986); A.A. Garren, D.E. Johnson, "The $90^{\circ}$ (September 1987) SSC Lattice," SSC Report SSC-146 (1987); Chapter 4 of the SCDR in Ref. 2.

36. A. Garren, A. Kenney, E. Courant, and M. Syphers, "A User's Guide to Synch," Fermi National Accelerator Laboratory Report FN-420 (1985).

37. D.C. Carey and F.C. Iselin, "A Standard Input Language for Particle Beam and Accelerator Computer Programs," Proc. 1984 Summer Study on the Design and Utilization of the Superconducting Supercollider, Snowmass, CO (June 1984).

38. R. B. Chaffee, "TopDrawer," SLAC Computation Group Note CGTM No. 178 (1980). 
39. A. Chao, "Recent Non-Linear Dynamics Studies for the SSC," AIP Conf. Proc., 230, 203, Y.H. Ichikawa and T. Tajima, eds. (1990).

40. F. Schmidt, private communication.

41. J. Irwin, private communication.

42. S.K. Kauffmann, D.M. Ritson, and Y.T. Yan, "Implementation of OneTurn Maps in SSCTRK using Zlib," SSC Laboratory Report SSCL-321 (1990).

43. J. Irwin, "Construction of High-order Maps for Large Proton Colliders," to appear in Proc. IEEE Part. Accel. Conf., San Francisco (May 1991).

44. T. Sen, Y. Yan, and J. Irwin, "Liemap-A Program for Extracting OneTurn Single Exponent Lie Generator Map," to appear in Proc. IEEE Part. Accel. Conf., San Francisco (May 1991).

45. Y. Yan, T. Sen, A. Chao, G. Bourianoff, A. Dragt, and E. Forest, "Comment on Round-off Errors and on One-Turn Taylor Maps," SSC Laboratory Report SSCL-301 (1990); Proc. Workshop on Nonlinear Problems in Future Particle Accelerators, Capri, Italy (April 1990).

46. A. Chan, T. Sen, Y. Yan, and E. Forest, "Applications of Zmap to the SSC," SSC Laboratory Report SSCL-459 (1991); to appear in Proc. IEEE Part. Accel. Conf., San Francisco (May 1991).

47. T. Sen, Y. Yan, A. Chao, and E. Forest, "Taylor Maps for Long-Term Tracking at the SSC," SSC Laboratory Report SSCL-497 (1991).

48. S.K. Kauffman, et al., work in progress.

49. Y. Yan, "Symplectic Factorization and Normalization of the Parameterized Courant-Snyder Matrix," SSC Laboratory Report SSCL-302 (1990).

50. L. Teng, private communication.

51. J. Ellison, private communication.

52. E. Forest, "Linear Floquet Normalization on the Matrix," unpublished.

53. J. Ellison and Y. Yan, work in progress.

54. Y. Yan, "Normalization of the Parameterized Courant-Snyder Matrix for Symplectic Factorization of a Parameterized Taylor Map," SSC Laboratory Report SSCL-460 (1990); to appear in Proc. IEEE Part. Accel. Conf., San Francisco (May 1991). 


\section{Disclaimer Notice}

This repon wes prepared as an account of work sponsored by an epency of the Unied Stales Government. Nether tho Unaed States Government or any epancy thered. nor eny of their

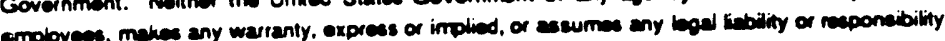

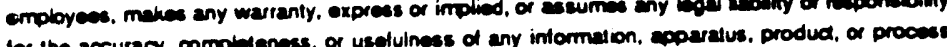

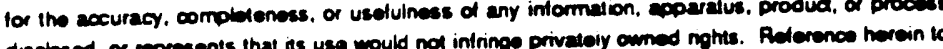

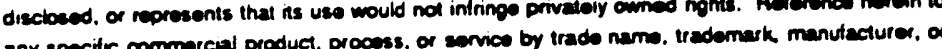

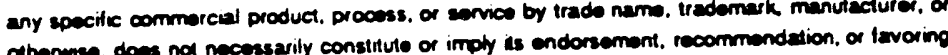
othormes. does nol necossavily constitute of imply is endorsement. recornmondelion, or lavoring by the United States Government of any apency thereof. The vews and coinions of authors aponcy thereot.

Superconducting Super Collider Laboratory is an equal opportunity employer. 

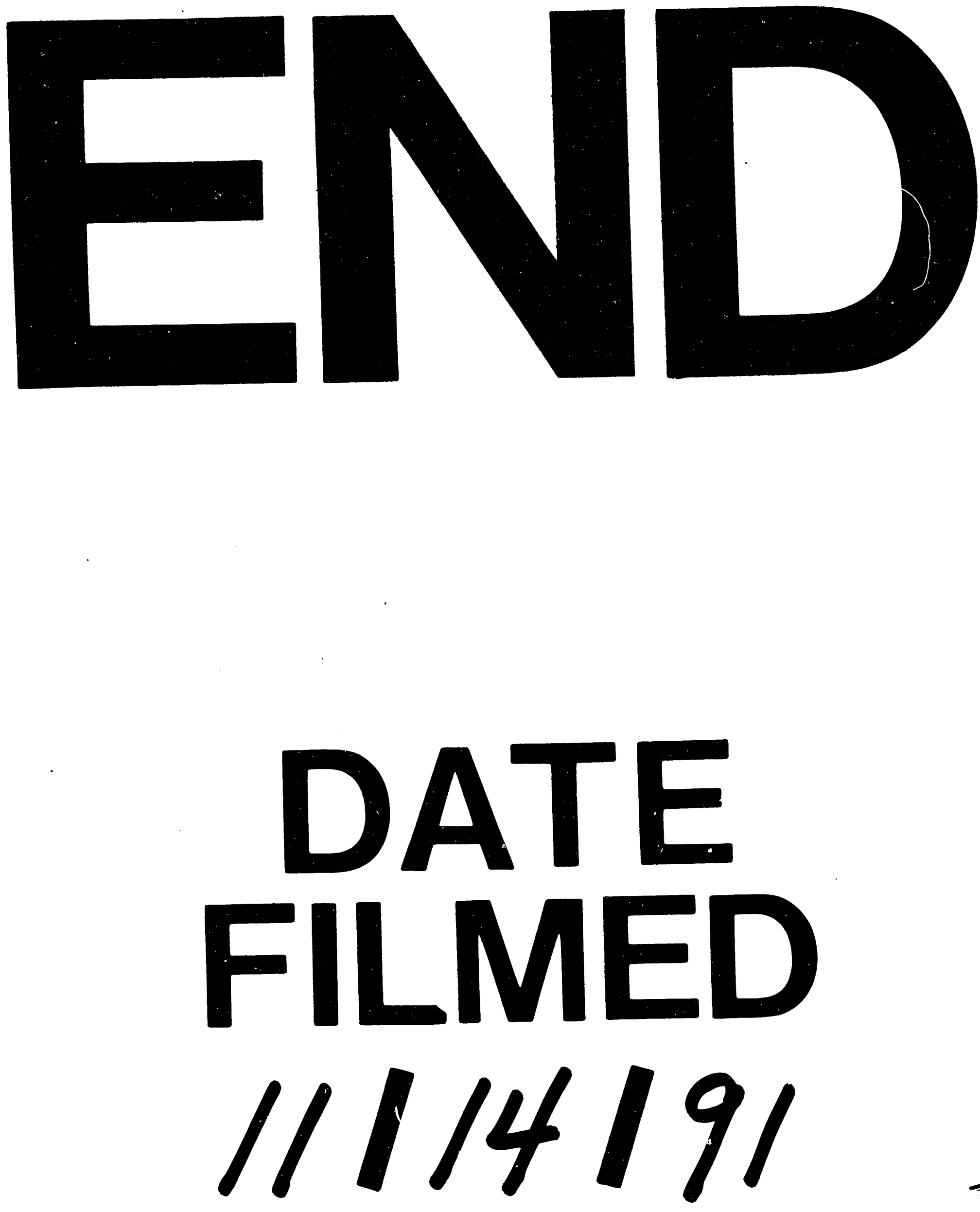

$I$ 
